\title{
Approach to a rational rotation number in a piecewise isometric system
}

\author{
J. H. Lowenstein and F. Vivaldi† \\ Dept. of Physics, New York University, 2 Washington Place, New York, NY 10003, USA \\ $\dagger$ School of Mathematical Sciences, Queen Mary, University of London, London E1 4NS, UK
}

\begin{abstract}
We study a parametric family of piecewise rotations of the torus, in the limit in which the rotation number approaches the rational value $1 / 4$. There is a region of positive measure where the discontinuity set becomes dense in the limit; we prove that in this region the area occupied by stable periodic orbits remains positive. The main device is the construction of an induced map on a domain with vanishing measure; this map is the product of two involutions, and each involution preserves all its atoms. Dynamically, the composition of these involutions represents linking together two sector maps; this dynamical system features an orderly array of stable periodic orbits having a smooth parameter dependence, plus irregular contributions which become negligible in the limit.
\end{abstract}

May 12, 2022

\section{Introduction}

This paper is devoted to the study of the action of the matrix

$$
C=\left(\begin{array}{cc}
\lambda & -1 \\
1 & 0
\end{array}\right) \quad \lambda=2 \cos (2 \pi \rho)
$$

on the unit square, in the limit $\lambda \rightarrow 0$. This deceptively simple dynamical system has a surprisingly complicated behaviour. In figure 2 we display the phase portrait in the North-East corner of the unit square, for $\lambda=2^{-6}$. The picture suggests the existence of an infinite hierarchy of stable islands, immersed in a 'pseudo-chaotic' sea.

More precisely, we let $\Omega=[0,1)^{2}$ and we define the map

$$
F: \Omega \rightarrow \Omega \quad(x, y) \mapsto(\lambda x-y+\iota(x, y), x) \quad \iota(x, y)=-\lfloor\lambda x-y\rfloor,
$$



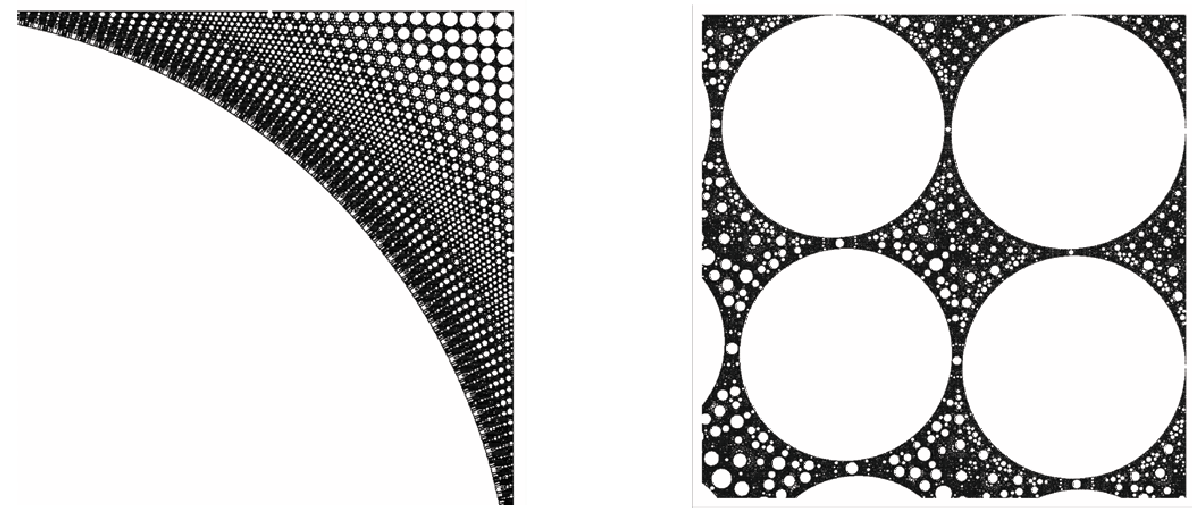

Figure 1: Left: Detail of the phase portrait of the map $F$ for $\lambda=2^{-6}$, corresponding to an irrational value of the rotation number $\rho$, close to $1 / 4$. A portion of the large central island appears in the SW corner of the picture. Right: Magnified view of the region near the NE corner.

where $\lfloor\cdot\rfloor$ denotes the floor function. This map is area-preserving and has time-reversal symmetry $[24]$

$$
F^{-1}=G \circ F \circ G^{-1} \quad G: \Omega \rightarrow \Omega \quad(x, y) \mapsto(y, x) .
$$

Occasionally, we will regard $F$ as a map of the torus, rather than of the square. It must be understood however, that the function $\iota$ in (2) is defined over $\Omega$, not over $\mathbb{R}^{2} / \mathbb{Z}^{2}$.

In the specified parameter range, the map $F$ is non-ergodic, and has zero topological entropy [8]. It is linearly conjugate to a piecewise rotation on a rhombus with rotation number $\rho$, and indeed it's a piecewise isometry with respect to the metric induced by the inner product

$$
\mathcal{Q}(U, V)=U_{x} V_{x}+U_{y} V_{y}-\frac{\lambda}{2}\left(U x V y+U_{y} V_{x}\right) .
$$

The quantity $\mathcal{Q}(U, U)$ is the invariant quadratic form of the matrix $C$ in (11). For $\lambda=0$, this metric reduces to the ordinary Euclidean metric. Throughout this paper, the term isometry will always refer to the metric (44). Piecewise isometries are dynamical systems that generalize to higher dimensions the construct of interval exchange maps. Recently, these systems have attracted a great deal of attention, both theoretically $[1,3,7,13-15,18$, $20,23,32]$, and in applications $[4,9-11,25,28,33]$.

In the analysis of these systems, a decisive simplifying factor is the presence of selfsimilarity, which allows a satisfactory - occasionally complete - characterization of the dynamics [30]. Self-similarity has been invariably found in maps with rational rotation number (the parameter $\rho$ in (11)), although the occurrence of scaling in this case has never been justified by a general theory. For rational rotations, the stable regions in phase space - the ellipses of figure 1- become convex polygons, and the system parameters (such as the quantity $\lambda$ in (1)) are algebraic numbers. Much of recent research has been devoted to quadratic parameter values, plus some scattered results for cubic parameters $[18,21,26,27,30]$. Rational rotation numbers with prime denominator were considered in [17] from a ring-theoretic angle, in a rather general setting. In all cases in which computations have been performed, the complement of the cells, namely the closure of 

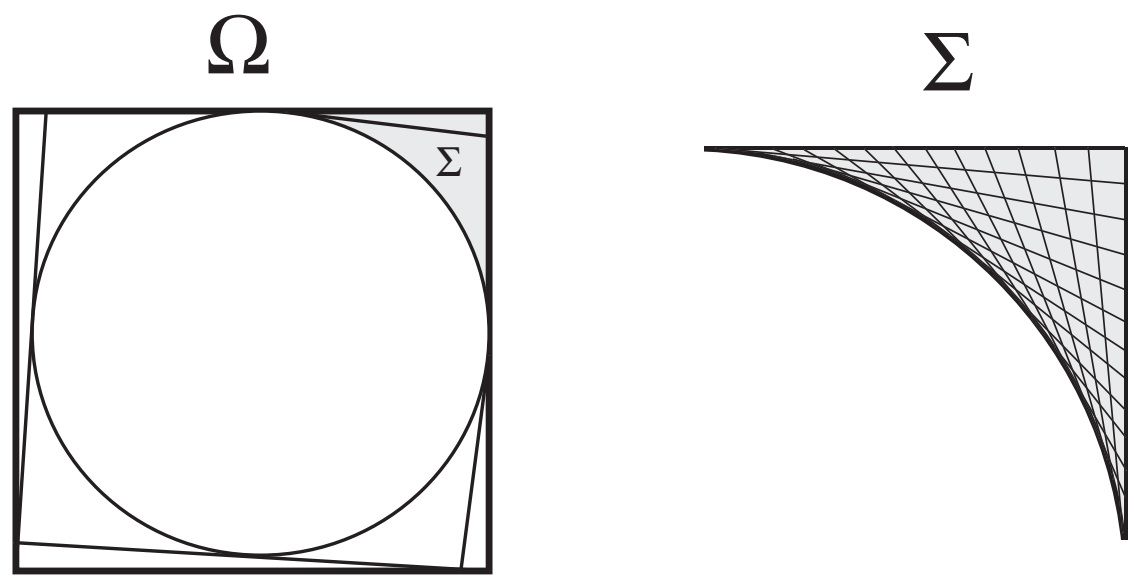

Figure 2: Left: Mismatch between the action of $F$ on $\Omega$ and a rotation by $\pi / 4$, for a parameter value close to zero. Shown are the first few images of the boundary of $\Omega$, which are tangent to the central island. Right: Dominant features of the discontinuity set in the North-East sector $\Sigma$.

the so-called discontinuity set (the dark area in figure 1), has been found to have zero Lebesgue measure. However, no general result has been established in this direction.

The case of irrational rotations - the generic one- has stubbornly resisted attack, largely due to an apparent lack of self-similarity. In 1997, P. Ashwin conjectured that for irrational rotations, the complement of the set of elliptic islands has positive Lebesgue measure, and this measure depends continuously on the parameter [2]. Subsequently, the semi-continuity of the measure at irrational rotation numbers was established rigorously [16]; however, the important question of positivity of measure remains unresolved, and constitutes the motivation for the present work. Ashwin's conjecture is analogous to the well-known conjecture of the positivity of measure of chaotic motions in Hamiltonian systems with divided phase space.

To identify elusive instances of scaling behaviour, in this paper we examine irrational rotations in the vicinity of a rational one. For values of the parameter $\lambda$ approaching zero, the map $F$ approaches a rotation of the unit square about its centre, by the angle $\pi / 2$ (figure 22). To a first approximation, the mismatch between the actual and the limit dynamics causes the images of the boundary of the square (the discontinuity set) slowly to build a regular envelope of the central disk, by line segments. These segment intersect transversally, forming an orderly array of pseudo-hyperbolic points [31]. It is not difficult to see that in the limit the pseudo-hyperbolic points become dense in the four corner sectors, and any stable island present in these sectors must be confined within the meshes of the grid, which are of vanishing size. On the other hand, at $\lambda=0$ the discontinuity set is just the boundary of the unit square, and all this complicated structure disappears.

This paper is devoted to the study of the limit $\lambda \rightarrow 0$. We have two main results. Firstly, we construct an induced mapping that describes in this limit the dynamics of $F$ outside the main island. This map has interesting properties, summarized in the following theorem. 
Theorem A. For $\lambda$ positive and sufficiently small, there is a mapping $L$, induced by $F$ on a domain $\Lambda$ of area $O(\lambda)$, which serves as a return map for all orbits outside the main island, apart from a set of measure $O\left(\lambda^{2}\right)$. The mapping $L$ is the composition of two involutions; each involution preserves all its atoms, apart from a set of irregular atoms of total measure $O\left(\lambda^{3}\right)$.

Theorem A is the summary of theorems 6 and 11, which describe the structure of the involutions comprising $L$, and theorem 23 , which estimates the measure of the orbits that pass through the so-called regular atoms of the map $L$. This result leads us to consider the symmetric fixed points of $L$, which form a distinguished family of stable periodic orbits of the map $F$ - the regular sequence. Our second result concerns the area of the corresponding stable regions.

Theorem B. As $\lambda \rightarrow 0^{+}$, the orbits of $F$ that correspond to the fixed points of $L$ form an infinite family of stable symmetric cycles, with the property that the total area of the associated islands approaches a positive limit.

The above theorem will appear in the text as theorems 17 and 22, which also give detailed information about these periodic orbits. The 'perturbative' approach, whereby irregular contributions of vanishing measure are neglected, is an essential element of our analysis, since the complexity of the neglected dynamics is considerable.

This paper is organised as follows. In section 2 we provide the basic definitions and constructs. In section 3 we first define the induced map $L$, and then provide a geometrical proof of the main part of theorem A (theorems 6and 11). This proof is centred around the idea of sector maps, continuous transformations of a neighbourhood of an island, which are close to the identity. We will show that the dynamics of $F$ is the result of linking together two sector maps (see figures 3 and 44).

In section 4, we provide an algebraic proof of theorem 11, and also derive explicit formulae for the atoms of the return maps. These will be essential for the subsequent analysis. In section [5 we prove theorem B. We begin by constructing the regular sequence of periodic points, which shape the phase space outside the main island (figure 1). These orbits are parametrized by pairs of atoms of the two involutions comprising the mapping $L$ of theorem A. Then we use the formulae developed earlier to establish some asymptotic properties of the atoms of the aforementioned involutions. With these formulae, we prove that, as $\lambda \rightarrow 0$, the regular sequence of stable periodic points of $L$ becomes infinite, and the limiting area of the ellipses associated with the regular sequence approaches a positive limit (theorem 22). Our proof is constructive, and we derive an analytic formula for the total area.

In section 6 we complete the proof of theorem A; we prove that the domain of definition of the induced map $L$-indeed the subset of it constituted by the regular atoms of $L$ - is a surface of section for all orbits, except for a set of measure $O\left(\lambda^{2}\right)$ (theorem 23). Finally, in section 7 we briefly consider the extension of our results to the case of negative $\lambda$ and to the dynamics of irregular atoms. Several formulae and proofs are collected in an appendix. 


\section{Preliminaries}

The map $F$ consists of the action of the matrix (10), followed by a translation by $\iota$, given in equation (2). The quantity $\iota$ takes a finite set of integer values. In particular

$$
\iota(x, y) \in\{0,1\} \quad \text { if } \quad 0 \leq \lambda<1 .
$$

We shall now assume that $\lambda$ belongs to this range, approaching zero from above; later on, we shall restrict the parameter further, to a smaller right neighbourhood of zero. The case of negative parameters will be considered briefly in section 7 .

The unit square $\Omega$ is partitioned into two atoms $\Omega_{i}$, which are convex polygons given by the level sets of the function $\iota$

$$
\Omega_{i}=\iota^{-1}(j) \quad j=0,1 .
$$

Given a code $1^{1} \iota=\left(\iota_{0}, \iota_{1}, \ldots\right)$, with integer symbols $\iota_{t}$ taken from the alphabet (5), we consider the set $\mathcal{C}(\iota)$ of the points $z \in \Omega$ for which $\iota\left(F^{t}(x, y)\right)=\iota_{t}$, for $t=0,1, \ldots$ These are the points whose images visit the atoms in the order specified by the code. If $\mathcal{C}(\iota)$ is non-empty, then it is called a cell. Generically, a cell is an open ellipse together with a subset of its boundary [16, proposition 2]. In coordinates relative to their centre, these ellipses are similar to the ellipse $\mathcal{Q}(x, y)=1$, cf. equation (4). A cell can also be a convex polygon (when the rotation number $\rho$ is rational), or a point.

The set of all images and pre-images of the boundary of the atoms constitutes the discontinuity set $\Gamma$

$$
\Gamma=\bigcup_{t=-\infty}^{\infty} F^{t}(\partial \Omega) \quad \partial \Omega=\bigcup_{i} \partial \Omega_{i}
$$

which consists of a countable set of segments. For the purpose of generating $\Gamma$, the set $\partial \Omega$ may be replaced with any set that covers $\partial \Omega$ under iteration. One verifies that the discontinuity set of the map (2) is generated by the oriented segment $\gamma$, with first endpoint $(0,0)$ (included) and second end-point $(0,1)$ (excluded). The map (2), viewed as a map of the torus, is discontinuous only on $\gamma$, from the left. Indeed

$$
F(1-x, y)-F(x, y)=(\lambda+\iota(1-x, y)-\iota(x, y), 1-2 x) \equiv(\lambda,-2 x)\left(\bmod \mathbb{Z}^{2}\right) .
$$

Letting $x \rightarrow 0^{+}$, we see that $F$ has a sliding singularity at $\gamma[22]$, which causes a shift by $\lambda$ in the horizontal direction. By time-reversal symmetry, the map $F^{-1}$ has a siding singularity on $G(\gamma)$.

The generator $\gamma$ is oriented in such a way that the map $F$ be continuous on the segment's right side. The images and pre-images of $\gamma$ inherit an orientation with the same property. In view of this, we'll say that a segment in the discontinuity set is glued to any domain tangent to it on the right. Furthermore, if the first and second end-points of a

\footnotetext{
${ }^{1}$ We use the symbol $\iota$ for both the coding function and the code.
} 
segment belong to two sets $A$ and $B$, respectively, we say that such a segment connects $A$ to $B$.

Necessarily, any periodic orbit of $F$ will have a point whose cell is tangent to $\gamma$, from either the left or the right. For $t \in \mathbb{Z}$, the set $F^{t}(\gamma)$ is a collection of segments. Given a cell $\mathcal{E}$, the regular component of $F^{t}(\gamma)$ with respect to $\mathcal{E}$ is the set of segments in $F^{t}(\gamma)$ that are tangent to $\mathcal{E}$. A non-empty regular component consists of a single segment; indeed all components of the image of $\gamma$ must have the same orientation, so that two tangent segments would have to lie at opposite points of $\mathcal{E}$. However, for $\lambda \neq 0$, there is no cell touching $\gamma$ on opposite sides, and the image of a tangent point is a single point. The same property holds also for images of the segment $G(\gamma)$, and indeed of any other segment. We will also speak of the regular component with respect to a cycle, because, given a cycle and an integer $t$, there is at most one ellipse in the cycle such that a component of $F^{t}(\gamma)$ is tangent to it. Regular components will be important in section [3, in the construction of the induced map.

At $\lambda=0$ the dynamics is trivial. The atom $\Omega_{0}$ (a triangle) collapses to the segment $G(\gamma)$, and the discontinuity set is just $\Gamma=\gamma \cup G(\gamma)$. All points in $\Gamma$ have period 2, except for a fixed point at the origin. The rest of the space - the interior of $\Omega$ - consists of a single square cell; its points have period four, except for a fixed point at the centre.

We are interested in periodic orbits for parameter values near zero. A periodic orbit $\left(\overline{x_{0}, x_{1}, \ldots, x_{t-1}}\right)$ corresponds to a periodic code $\iota=\left(\overline{\iota_{0}, \iota_{1}, \ldots, \iota_{t-1}}\right)$. As the parameter varies, the points of the orbit move in phase space; in [31, theorem 2] it was shown that the coordinates $x_{j}$ are rational functions of $\lambda$. Specifically, one has

$$
x_{j}(\lambda, \iota)=\frac{\mathcal{X}_{t}\left(\lambda, \sigma^{j}(\iota)\right)}{\mathcal{M}_{t}(\lambda)} \quad j=0, \ldots, t-1,
$$

where $\mathcal{X}_{t}$ and $\mathcal{M}_{t}$ are polynomials in $\mathbb{Z}[\lambda]$, and $\sigma$ is the left shift map. The denominator $\mathcal{M}_{t}$ is monic of degree $\lfloor(t+2) / 2\rfloor$ and it depends only on the period; the numerator $\mathcal{X}_{t}$ has degree $\lfloor(t+1) / 2\rfloor$, and it depends on the orbit via the code $\iota$. This algebraic structure is common for periodic points of piecewise affine systems, see, e.g., $[5,6]$. In section 5 we will derive ad hoc expressions for these functions, tailored for the $\lambda \rightarrow 0$ regime.

The symbolic dynamics is far from being complete, so not all periodic codes $\iota$ correspond to an actual periodic orbit. For this to be the case, the value of all functions (6) must belong to the half-open interval $[0,1)$. The function that is closest to the boundary of the unit interval determines the common size of all cells of the orbit corresponding to that code. Accordingly, we define the radius $r$ of a periodic orbit as

$$
r(\lambda)=\min _{0 \leq j \leq t-1}\left\{x_{j}(\lambda), 1-x_{j}(\lambda)\right\} .
$$

The function $r$ is piecewise rational; besides the singularities inherited from the functions $x_{j}$, the radius will typically have points with discontinuous first derivative, due to a change of the index $j$ for which the minimum in (7) is attained. An orbit exists if it has positive radius, and the radius becomes zero at the bifurcation parameter values. An orbit of zero radius exists if and only if none of its rational functions assume the value 1 , which is the excluded point in the unit interval. On the torus, this distinction becomes irrelevant. 
Formulae of the type (6) also describe the parameter dependence of pseudo-hyperbolic points [31, theorem 6]. These are the points that recur to the boundary of the atoms. More precisely, a point in $\Omega$ is pseudo-hyperbolic if it maps to $\gamma$ in the forward time direction, and to $G(\gamma)$, in the backward time direction. For an irrational rotation number, these points correspond to transversal intersections of two segments of the discontinuity set, which act like 'pseudo-separatrices'. It can be shown that, generically, an orbit on the discontinuity set can contain only finitely many pseudo-hyperbolic points, the first of which is in $G(\gamma)$, and the last in $\gamma$. A pseudo-hyperbolic point can be periodic only for parameter values which are algebraic of degree greater than one [31, theorem 9]. Thus, in general, a pseudo-hyperbolic sequence belongs to an infinite orbit; such a sequence is determined by a finite symbolic dynamics, which describes the itinerary between the endpoints on $G(\gamma)$ and $\gamma$.

Pseudo-hyperbolic points will occur as vertices of the atoms of the return map $L$, to be defined in the next section. As with periodic orbits, we shall derive explicit formulae for these points, appropriate for the $\lambda \rightarrow 0$ limit.

\section{Return map}

In this section we prove the main part of theorem A. We will construct an induced mapping $L$ that describes the dynamics of $F$ in the limit $\lambda \rightarrow 0^{+}$. This map is the composition of two involutions on a $\lambda$-dependent domain whose area vanishes in the limit (theorems 6 and 11).

From the time-reversal symmetry (3), we find that $F$ can be written as the composition of two orientation-reversing involutions

$$
F=H \circ G \quad \text { with } \quad H=F \circ G, \quad G^{2}=H^{2}=I d
$$

with $H$ given by $(x, y) \mapsto(\{\lambda y-x\}, y)$, where $\{\cdot\}$ denotes the fractional part. Let Fix $G$ and Fix $H$ be the sets of fixed points of the involutions $G$ and $H$, respectively. The set Fix $G$ is the segment $x=y$, independent of $\lambda$; for $0 \leq \lambda<1$, the set Fix $H$ consists of two segments, with end-points $(0,0),(\lambda / 2,1)$, and $(1 / 2,0),((\lambda+1) / 2,1)$, respectively.

We begin by examining the rational functions of the main periodic orbits of $F$ (cf. equation (6) )

\begin{tabular}{lllcl}
$t$ & code & denominator & numerators & radius \\
\hline 1 & $(\overline{1})$ & $2-\lambda$ & $(1)$ & $1-x_{0}(\lambda)$ \\
2 & $(\overline{01})$ & $4-\lambda^{2}$ & $(2, \lambda)$ & $x_{1}(\lambda)$
\end{tabular}

These cycles are symmetric ( $G$-invariant), and the 2-cycle belongs to Fix $H$. At $\lambda=0$ the fixed point has radius $1 / 2$, while the 2 -cycle has zero radius, but it still exists on the discontinuity set: $\{(1 / 2,0),(0,1 / 2)\}$. It turns out that, in the $\lambda \rightarrow 0^{+}$limit, the dynamics is dominated by these two periodic orbits, which determine the coarse features of the 
phase space. The fine structure also depends on other periodic orbits, some of which will be analysed in section 7 .

The ellipse $\mathcal{E}$ bounding the large cell of the 1-cycle is tangent to the segment $\gamma$ at the point $(1, \tau)$, where $\tau=(1+\lambda) / 2$. By $G$-symmetry, $\mathcal{E}$ is also tangent to $\gamma_{0}=G(\gamma)$ at the point $T_{0}=(\tau, 1)$. The open region delimited by the segments $\gamma, \gamma_{0}$, and the elliptical arc connecting $T_{0}$ to $G\left(T_{0}\right)$ clockwise will be called the 1-sector, denoted by $\Sigma$. By construction, the set $\Sigma$ is invariant under $G$. The 1-sector -indeed a vanishingly small subset of it - will serve as a surface of section for orbits outside the main island. Not all such orbits intersect $\Sigma$, for example, the 2-cycle displayed in (9), and the cycles mentioned in section 7 . However, the missing orbits will be shown to have vanishing measure. More important, the distinction between the orbits that enter $\Sigma$ and those that don't will be essential to our analysis.

For $\lambda$ near zero, the map $F^{4}$ is close to the identity, and hence the set $\Sigma$ is 'close to' being invariant under $F^{4}$. There are however orbits that enter and leave $\Sigma$; to characterize them, we define the sets

$$
\Lambda=\Sigma \backslash F^{4}(\Sigma) \quad \Xi=F^{4}(\Sigma) \backslash \Sigma
$$

where, by time-reversal symmetry,

$$
G(\Lambda)=\Sigma \backslash F^{-4}(\Sigma) \quad \Xi=F^{4} \circ G(\Lambda) .
$$

Thus $G(\Lambda)$ is the set of points that leave $\Sigma$ under $F^{4}$. Below, we will define two induced maps over the set $\Lambda$.

To determine the set $\Lambda$, we construct the first few images of the segment $\gamma$, which are listed in table 1 below as pairs of end-points. We adopt the following conventions: $i$ ) an integer $\mathbf{n}$ in boldface denotes the $n$-th iterate of the generator $\gamma\left(\right.$ e.g., $\left.\mathbf{3}=F^{3}(\gamma)\right)$; $\left.i i\right)$ segments crossing $\gamma$ have negative $x$-coordinate in the end-point on the left of $\gamma$; iii) if an end-point of $\mathbf{n}$ belongs to more than one set $\mathbf{m}$, we list only the value of $m$ corresponding to the lowest order iterate.

\begin{tabular}{cll} 
segment & $\begin{array}{l}\text { first } \\
\text { end-point }\end{array}$ & $\begin{array}{l}\text { second } \\
\text { end-point }\end{array}$ \\
\hline $\mathbf{0}$ & $(0,0)$ & $(0,1)$ \\
$\mathbf{1}$ & $(1,0) \in \mathbf{0}$ & $(0,0) \in \mathbf{0}$ \\
$\mathbf{2}$ & $(\lambda, 1) \in \mathbf{1}$ & $(0,0) \in \mathbf{0}$ \\
$\mathbf{3}$ & $\left(\lambda^{2}, \lambda\right) \in \mathbf{2}$ & $(1,0) \in \mathbf{0}$ \\
$\mathbf{4}$ & $\left(-\lambda+\lambda^{3}, \lambda^{2}\right) \in \mathbf{3}$ & $(\lambda, 1) \in \mathbf{1}$ \\
$\mathbf{5}^{1}$ & $\left(\lambda-2 \lambda^{2}+\lambda^{4}, 1-\lambda+\lambda^{3}\right) \in \mathbf{4}$ & $(g-1,1) \in \mathbf{1}$ \\
$\mathbf{5}^{2}$ & $\left(g^{\prime}, 0\right) \in \mathbf{1}$ & $\left(\lambda^{2}, \lambda\right) \in \mathbf{2}$ \\
& & \\
& $g=\frac{1+2 \lambda-\lambda^{2}-\lambda^{3}}{2-\lambda^{2}}$ & $g^{\prime}=\frac{1-\lambda^{2}}{2-\lambda^{2}}$.
\end{tabular}




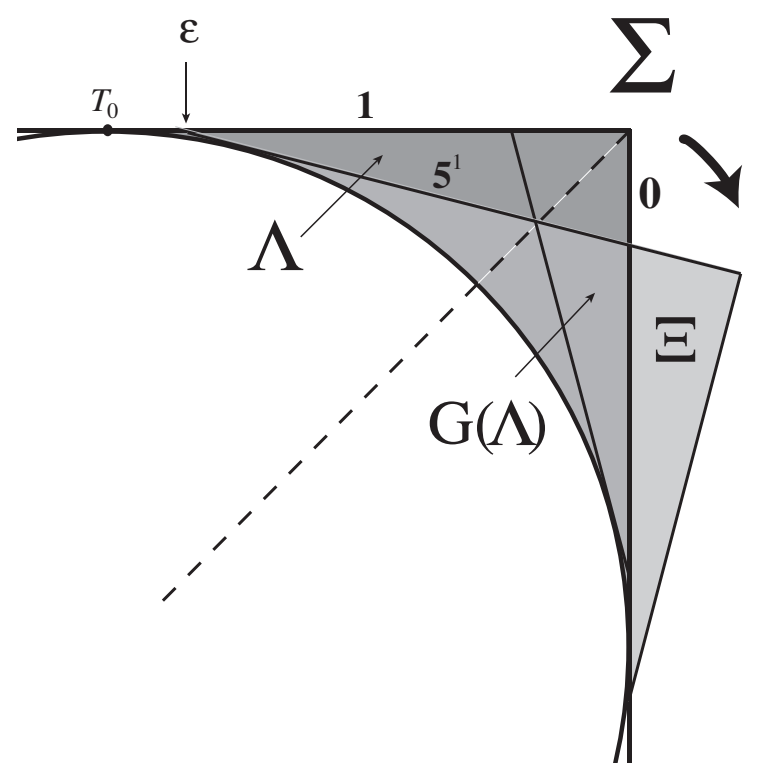

Figure 3: The sector $\Sigma$, and its image under $F^{4}$, corresponding to a small clockwise rotation. The darker region is the entry sub-domain $\Lambda$; the exit sub-domain $G(\Lambda)$ is the reflection of $\Lambda$ with respect to the symmetry axis Fix $G$ (the dashed line). All points in $\Sigma$ will eventually map to the region $\Xi=F^{4} \circ G(\Lambda)$, located on the opposite side of the discontinuity line $\mathbf{0}$.

Table 1: The first five iterates of the generator $\gamma$ of the discontinuity set.

The segments $\mathbf{0}, \ldots, \mathbf{4}$ are regular components with respect to both the 1-cycle and the 2-cycle. Since $0 \leq \lambda<1$, the segment 4 crosses the discontinuity line $\gamma$, and so its image consists of two segments $\mathbf{5}^{1}$ and $\mathbf{5}^{2}$. These are the images of the portions of $\mathbf{4}$ lying to the left and to the right of $\gamma$, respectively, and they are regular components of $\mathbf{5}$ with respect to the 1 -cycle and the 2 -cycle, respectively.

We define approximate orthogonality relations. If the angle between two segments $\mathbf{m}$ and $\mathbf{n}$ is equal to $0, \pi+O(\lambda)$, we say that $\mathbf{m}$ and $\mathbf{n}$ are quasi-parallel, and write $\mathbf{m} \| \mathbf{n}$. Likewise, two segments are quasi-perpendicular $(\mathbf{m} \perp \mathbf{n})$ if the angle between them is $\pm \pi / 2+O(\lambda)$. Plainly, $\mathbf{m}$ and $\mathbf{n}$ are quasi-parallel (perpendicular) if $m-n$ is even (odd).

There are also incidence relations. We use the notation $\mathbf{m} \dashv \mathbf{n}$ to indicate that one endpoint of $\mathbf{m}$ lies in the interior of $\mathbf{n}$ (and similarly for $\mathbf{m} \vdash \mathbf{n}$ ).

The images of $\gamma$ have the following behaviour under symmetry

$$
\begin{aligned}
& G(\mathbf{m})=G \circ F^{m}(\gamma)=F^{-m} \circ G(\gamma)=F^{-m} \circ F(\gamma)=-\mathbf{m}+\mathbf{1} \\
& H(\mathbf{m})=H \circ F^{m}(\gamma)=H \circ H \circ G \circ F^{m-1}(\gamma)=G \circ F^{m-1}(\gamma)=-\mathbf{m}+\mathbf{2} .
\end{aligned}
$$


We specify polygons/sectors by listing their boundary components between angle brackets, arranged clockwise, e.g.,

$$
\Omega_{0}=\langle\mathbf{0}, \mathbf{1},-\mathbf{1}\rangle \quad \Sigma=\langle\mathcal{E}, \mathbf{1}, \mathbf{0}\rangle .
$$

Since $G$ and $H$ are orientation-reversing, mapping a polygon entails mapping its sides, and reversing their order, e.g.,

$$
G\left(\Omega_{0}\right)=\langle G(\mathbf{0}), G(-\mathbf{1}), G(\mathbf{1})\rangle=\langle\mathbf{1}, \mathbf{2}, \mathbf{0}\rangle .
$$

The segment $\mathbf{5}^{1}$ connects $\mathbf{4}$ to $\mathbf{1}$, and $\mathbf{5}^{1} \cap \Sigma$ is the segment with end-points

$$
\left(1, g^{\prime \prime}\right),(g, 1) \quad g^{\prime \prime}=\frac{1}{1+\lambda-\lambda^{2}}
$$

where $g$ was given above. As $\lambda$ approaches zero, $g \rightarrow 1 / 2$ and $g^{\prime \prime} \rightarrow 1$. For our purpose it is sufficient to require that $g^{\prime \prime}<g$; so we replace the bounds in (5) by the more restrictive conditions

$$
0<\lambda<\lambda_{+}<1 \quad \text { where } \quad \lambda_{+}=2 \cos (2 \pi / 9) .
$$

(The number $\lambda_{+}$is algebraic, being a root of the polynomial $x^{3}-3 x+1$.)

The segment $5^{1}$ is tangent to the ellipse $\mathcal{E}$ at the point $T_{1}$; hence $\mathbf{5}^{1}$ decomposes $\Sigma$ into three regions (figure 3); a large triangular sector $F^{4}(\Sigma) \cap \Sigma$, of area $O(1$ ), a right triangle of area $O(\lambda)$, and a small triangular sector $\varepsilon$ of area $O\left(\lambda^{3}\right)$. The set $\Lambda$ is the union of the last two regions. We find

$$
\Lambda=\left\langle\mathbf{0}, \mathbf{5}^{1}, \mathbf{1}\right\rangle \cup \varepsilon \quad \Xi=\left\langle\mathbf{0}, \mathbf{5}^{1}, \mathbf{4}\right\rangle .
$$

Our analysis will be perturbative, and in what follows we shall neglect domains of area $O\left(\lambda^{2}\right)$. In particular, we shall omit $\varepsilon$ from all considerations, and represent $\Lambda$ as a triangle.

We intend to study the dynamics outside the main island via the first return map $L$ induced by $F$ on $\Lambda$. This map is constructed as the composition of two transit maps

$$
L=\bar{L}^{\text {out }} \circ \bar{L}^{\text {in }} \quad \bar{L}^{\text {in }}: \Lambda \rightarrow G(\Lambda) \quad \bar{L}^{\text {out }}: G(\Lambda) \rightarrow \Lambda
$$

where $\bar{L}^{\text {in }}$ and $\bar{L}^{\text {out }}$ are the first-return maps induced by $F$ on the respective sets. The map $\bar{L}^{\text {in }}$ is built up from iterations of $F^{4}$ inside $\Sigma$, the map $\bar{L}^{\text {out }}$ is built up (after the initial step out of $\Sigma$ ) by the iteration of $F$ outside $\Sigma$.

The definition of the maps $\bar{L}^{\text {in,out }}$ requires some care. As noted above, some orbits outside the main island do not enter $\Sigma$ at all, and it is conceivable that some orbits exiting $\Sigma$ will never return there. From Poincaré recurrence theorem, we know that these points have zero measure; so we'll ignore their contribution and accept that the map $\bar{L}^{\text {out }}$ may be undefined on a zero measure subset of $G(\Lambda)$. In particular, the transit time of the map $\bar{L}^{\text {out }}$ may be unbounded. The same is true for $\bar{L}^{\text {in }}$, as a result of having dropped the small set $\varepsilon$ from the domain $\Lambda$.

The following result has important consequences for the dynamics of the sector $\Sigma$. 
Lemma 1 The restriction to the sector $\Sigma$ of the maps $F^{ \pm 4}$ is continuous. Continuity extends to the boundary of $\Sigma$, apart from a sliding singularity of $F^{4}$ on $\gamma$, and of $F^{-4}$ on $G(\gamma)$. The symbolic dynamics is $(1,1,1,1)$ for points in $\Sigma \backslash G(\Lambda)$ and $(1,1,1,0)$ for points in $G(\Lambda)$.

Proof. Since $F$ is continuous away from $\gamma$, and $\gamma=\mathbf{0}$ is a sliding singularity (the segment $\mathbf{0}$ does not detach itself from $\Sigma$ under iteration) we have

$$
F^{t}(\Sigma)=\langle\mathcal{E}, \mathbf{t}+\mathbf{1}, \mathbf{t}\rangle, \quad t=0, \ldots, 3 \quad F^{4}(\Sigma)=\left\langle\mathcal{E}, \mathbf{5}^{1}, \mathbf{4}\right\rangle .
$$

From table 1, we find that, in the parameter range (15), of the first four images of $\mathbf{0}$, only 4 intersect $\gamma$. However, the point at which $\mathbf{4}$ is tangent to $\mathcal{E}$ lies to the left of $\gamma$. Hence none of the first three images of $\Sigma$ intersects $\gamma$, and therefore $F^{4}$ is continuous on $\Sigma$. The continuity of the inverse follows from time-reversal symmetry. From (18), we find that $F(\Sigma) \cap \Sigma=\emptyset$, and hence all points in $\Sigma \backslash G(\Lambda)$ return to $\Sigma$ in four iterations of $F$ and no fewer, with return symbolic dynamics $(1,1,1,1)$. Furthermore, from (13) and (16), we have $G(\Lambda)=\langle\mathbf{- 4}, \mathbf{1}, \mathbf{0}\rangle$, and since $G(\Lambda) \subset \Sigma$ and $F^{3}$ is continuous there, we have $F^{3}(G(\Lambda))=\langle-\mathbf{1}, \mathbf{4}, \mathbf{3}\rangle \subset \Omega_{0}$. So the symbolic dynamics of a point in $G(\Lambda)$ is $(1,1,1,0)$.

The transit maps $\bar{L}^{\text {in,out }}$ are piecewise isometries, and our next step is to partition their respective domains $\Lambda$ and $G(\Lambda)$ into atoms. Since the two domains are mirror image of each other under the involution $G$ (see equation (10)), we replace (17) by the more convenient decomposition

$$
L=L^{\text {out }} \circ L^{\text {in }} ; \quad L^{\text {in,out }}: \Lambda \rightarrow \Lambda, \quad L^{\text {in }}=G \circ \bar{L}^{\text {in }}, \quad L^{\text {out }}=\bar{L}^{\text {out }} \circ G
$$

where $\Lambda$ is the common domain of the two maps. We will often represent the right triangle $\Lambda$ using scaled coordinates, with the shorter leg being four times the length of the longest one - see figure 7 .

The distinction between regular and irregular atoms will be important in what follows. All regular atoms share a simple geometrical structure (apart from two exceptions), determined by regular components with respect to a periodic island. Asymptotically, these atoms occupy the entire domain $\Lambda^{\text {in }}$ of definition of the maps. Irregular atoms are more varied, but their detailed description is not required in asymptotic calculations, because in the limit, their total area becomes negligible.

\subsection{Preliminary lemmas}

The proof of theorem A will be quite laborious. We begin with some preliminary lemmas on involutivity and symmetry. We call an atom maximal if it is not properly contained in another atom.

Lemma 2 Let $L$ be a piecewise isometric involution. Then the image of a maximal atom is an atom. In particular, a maximal atom containing a fixed point is invariant. 
Proof. Since $L$ is an involution, its atoms are the same as those of $L^{-1}$. If $\Omega$ is an atom of $L$, we claim that $L$ is continuous on $L(\Omega)$. Indeed if it were not, we could find arbitrarily close points $z, w \in L(\Omega)$ mapping far apart in $\Omega$, thereby contradicting the fact that $L$ is an isometry on $\Omega$. Thus $L(\Omega) \subset \Omega^{\prime}$, where $\Omega^{\prime}$ is another atom. If this inclusion were proper, then $L\left(\Omega^{\prime}\right)$ would be a connected set properly containing $\Omega$, because $L$ is continuous on $\Omega^{\prime}$ and volume-preserving. But since $L$ is continuous on $L\left(\Omega^{\prime}\right)$, then $\Omega$ would not be maximal. So, if $\Omega$ is maximal, then $L(\Omega)=\Omega^{\prime}$, as desired. Furthermore, if $\Omega$ contains a fixed point of $L$, then $\Omega$ intersects $L(\Omega)$, and is therefore invariant.

Lemma 3 The maps $L^{\text {in,out }}$ are involutions.

Proof. Let $z \in \Lambda$. Since $\bar{L}^{\text {in }}$ is an induced map, we have $\bar{L}^{\text {in }}(z)=F^{t}(z)$, for some $t=t(z)$. From (3), a straightforward induction gives $F^{-t}=G \circ F^{t} \circ G$, for all $t \in \mathbb{Z}$, and hence

$$
L^{\text {in }} \circ L^{\text {in }}(z)=G \circ F^{t} \circ G \circ F^{t}(z)=F^{-t} \circ F^{t}(z)=z .
$$

Since $z$ was arbitrary, $L^{\text {in }}$ is an involution. The involutive nature of $L^{\text {out }}$ is proved with an analogous argument.

Next we look at the fixed sets of these involutions.

Lemma 4 Let $\Lambda_{k}^{\text {in,out }}$ be an atom of $L^{\text {in,out }}$, and let $t=t(k)$ be its transit time (number of iterations of $F$ ). Then

$$
\begin{array}{lll}
\text { Fix } L_{k}^{\text {in }}=F^{-s}(\operatorname{Fix} G) \cap \Lambda_{k}^{\text {in }} & \text { if } & t=2 s \\
\text { Fix } L_{k}^{\text {in }}=F^{-s} \circ G(\operatorname{Fix} H) \cap \Lambda_{k}^{\text {in }} & \text { if } & t=2 s+1 \\
\operatorname{Fix} L_{k}^{\text {out }}=F^{s}(\operatorname{Fix} G) \cap \Lambda_{k}^{\text {out }} & \text { if } & t=2 s \\
\operatorname{Fix} L_{k}^{\text {out }}=F^{s}(\operatorname{Fix} H) \cap \Lambda_{k}^{\text {out }} & \text { if } & t=2 s+1
\end{array}
$$

where $L_{k}^{\text {in,out }}$ is the restriction of $L^{\text {in,out }}$ to $\Lambda_{k}^{\text {in,out }}$.

Proof. From (19), the fixed points equations become

$$
L^{\text {in }}(z)=z \Leftrightarrow G \circ F^{t}(z)=z \quad L^{\text {out }}(z)=z \Leftrightarrow F^{t} \circ G(z)=z
$$

and we are interested in solutions (if any) within $\Lambda_{k}^{\text {in,out }}$. First, we consider the inner map $L^{\text {in }}$, and $z \in \Lambda_{k}^{\text {in }}$. If $t=2 s$, equation (20) becomes

$$
\begin{aligned}
G \circ F^{2 s}(z)=z & \Leftrightarrow F^{-2 s} \circ G(z)=z \\
& \Leftrightarrow F^{-s} \circ G(z)=F^{s}(z) \\
& \Leftrightarrow G \circ F^{s}(z)=F^{s}(z) .
\end{aligned}
$$


This shows that $F^{s}(z) \in \operatorname{Fix} G$, namely that $z \in F^{-s}(\operatorname{Fix} G)$. If $t=2 s+1$ is odd, we obtain, using (8)

$$
\begin{aligned}
G \circ F^{2 s+1}(z)=z & \Leftrightarrow F^{-(s+1)} \circ G(z)=F^{s}(z) \\
& \Leftrightarrow F \circ F^{s}(z)=G \circ F^{s}(z) \\
& \Leftrightarrow H \circ G \circ F^{s}(z)=G \circ F^{s}(z) .
\end{aligned}
$$

We find that $G \circ F^{s}(z) \in$ Fix $H$, or $z \in F^{-s} \circ G($ Fix $H)$.

Similarly, for $z \in \Lambda_{k}^{\text {out }}$, and $t$ even, the outer map gives

$$
\begin{aligned}
F^{2 s} \circ G(z)=z & \Leftrightarrow F^{s} \circ G(z)=F^{-s}(z) \\
& \Leftrightarrow G \circ F^{-s}(z)=F^{-s}(z),
\end{aligned}
$$

so $F^{-s}(z) \in$ Fix $G$, that is, $z \in F^{s}($ Fix $G)$. Finally, if $t$ is odd

$$
\begin{aligned}
F^{2 s+1} \circ G(z)=z & \Leftrightarrow F^{s+1} \circ G(z)=F^{-s}(z) \\
& \Leftrightarrow G \circ F^{-1} \circ F^{-s}(z)=F^{-s}(z) \\
& \Leftrightarrow H \circ F^{-s}(z)=F^{-s}(z)
\end{aligned}
$$

which gives $F^{-s}(z) \in$ Fix $H$, that is, $z \in F^{s}($ Fix $H)$.

We will show that for a significant class of atoms, the transit times of $L^{\text {in }}$ and $L^{\text {out }}$ are even and odd, respectively, and the fixed lines of the involutions intersect these atoms, which are themselves invariant. This will be done in the next two sections, where we look in detail at the transit maps.

\subsection{Structure of $L^{\text {in }}$}

The map $L^{\text {in }}$ results from restricting to $\Sigma$ the action of the matrix $C^{4}$ (see equation (1)) with respect to the fixed point of $F$. We consider again the point $T_{0}$ at which the ellipse $\mathcal{E}$ is tangent (on the torus) to the segment $\mathbf{1}$, see figure 3 , Let $T_{1}, T_{2}, \ldots$ be the images of $T_{0}$ under $F^{4}$, and let

$$
N=\left\lfloor\frac{1}{4(1-4 \rho)}-\frac{1}{4}\right\rfloor \quad \rho=\frac{1}{2 \pi} \cos ^{-1}(\lambda / 2)
$$

where $\rho$ is the rotation number, see equation (11). Then the first $N$ points of this sequence lie on the ellipse $\mathcal{E}$, within the boundary of $\Sigma$.

For $n=0, \ldots, N-1$, we let $\gamma_{n}=(\mathbf{4 n}+\mathbf{1})^{1}$ be the regular component of $F^{4 n}(\mathbf{1})$ with respect to $\mathcal{E}$. Then $\gamma_{n}$ is tangent to $\mathcal{E}$ at the point $T_{n}$. For any $k, \gamma_{n}$ and $\gamma_{n+k}$ are quasi-parallel, and their orientations agree. The segments $\gamma_{n}$ will be used to construct the atoms of $L^{\text {in }}$. We begin with a lemma, whose geometric content is illustrated in figure 5 . 


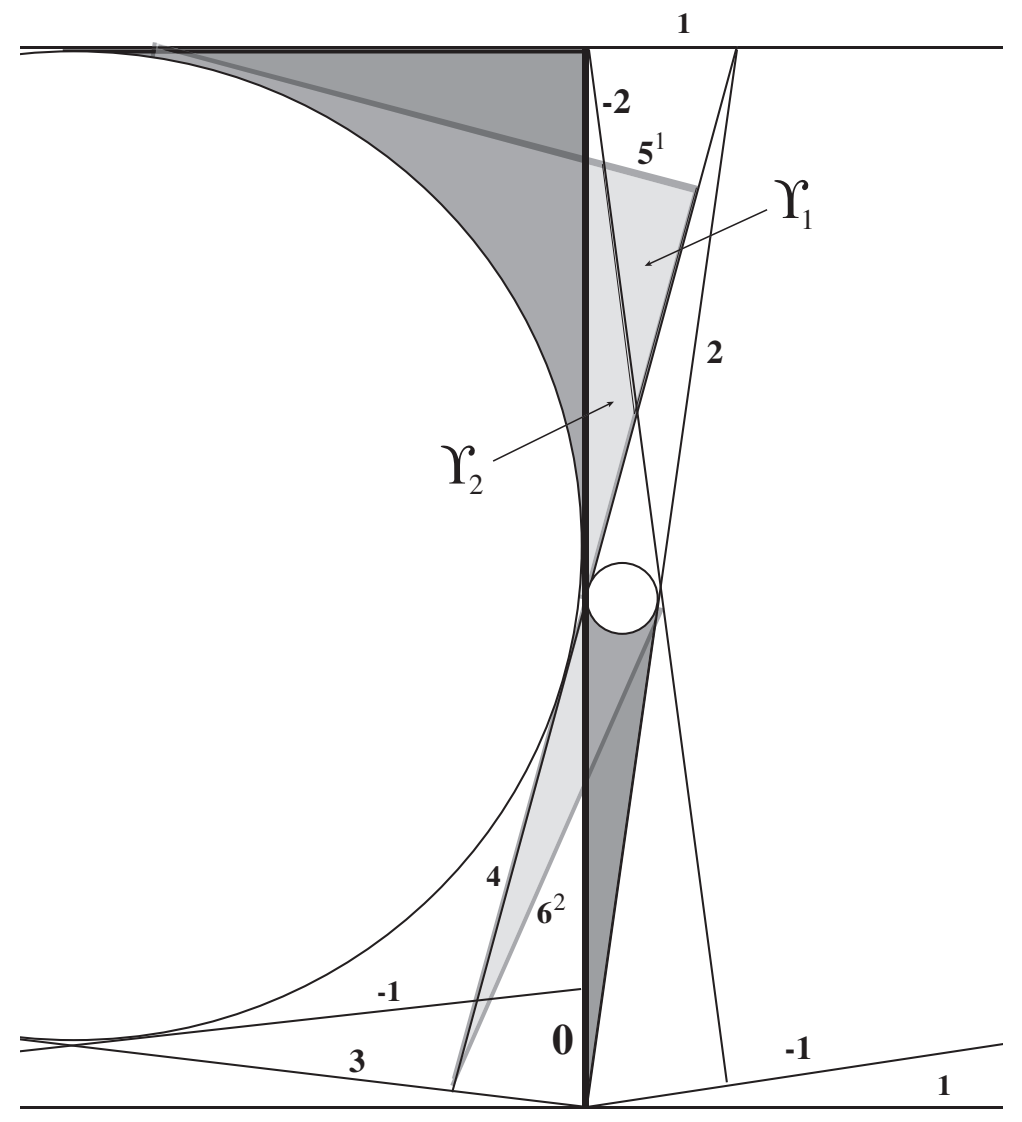

Figure 4: The linked sector maps of the 1-cycle (the large circle) and the 2-cycle (the small circle), which lie on opposite sides of the discontinuity line $\mathbf{0}$. Under the action of the map $F^{4}$, part of the sectors $\Sigma$ and $\Sigma^{\prime}$ (the darker regions) escape to the triangles $\Xi$ and $\Xi^{\prime}$, respectively, located on the opposite side of $\mathbf{0}$. The points in $\Xi$ that do not end up in $\Sigma^{\prime}$ (under $F^{2}$ ) comprise the triangle $\Upsilon_{1}$; those that do comprise the quadrilateral $\Upsilon_{2}$.

Lemma 5 For $n=1, \ldots, N-1$, the regular component $\gamma_{n}$ connects 4 to $\gamma_{n-1}$, and it intersects transversally the segments $\mathbf{0}$ and $-\mathbf{4}^{1}$ on the boundary of $G(\Lambda)$, apart from a possible non-generic degeneracy of $\gamma_{N-1} \cap-\mathbf{4}^{1}$ into a segment.

Proof. From (16) we verify that $\mathbf{0}$ and $-\mathbf{4}^{1}$ do indeed belong to the boundary of $G(\Lambda)$. Let $n=1$. The segment $\gamma_{1}=\mathbf{5}^{1}$ connects $\mathbf{4}$ to $\gamma_{0}=\mathbf{1}$ - see table 1. For the parameter $\lambda$ in the range (15), the point $T_{1}$ lies outside $G(\Lambda)$, and therefore $\gamma_{1}$ crosses 0 and $-\mathbf{4}^{1}$ transversally. We proceed by induction on $n$, and assume that for some $n$ in the range $1 \leq n<N-1$, the segment $\gamma_{n}$ connects 4 to $\gamma_{n-1}$, and it is tangent to $\mathcal{E}$ at a point $T_{n}$ lying outside $G(\Lambda)$. Then $\gamma_{n}$ intersects $\gamma$ transversally, so we consider the segment $\gamma_{n}^{-}=\gamma_{n} \cap \Sigma$. By construction, the segment $\gamma_{n}^{-}$connects $\gamma$ to $\gamma_{n-1}$. From lemma 1, the set $\gamma_{n+1}=F^{4}\left(\gamma_{n}^{-}\right)$is a segment tangent to $\mathcal{E}$ at a point $T_{n+1}$, which connects 4 to $\gamma_{n}$ (the statement concerning the first end-point is justified by the sliding nature of the singularity on $\gamma$ ). Since $F^{4}$ is close to the identity, the orientations of $\gamma_{n}$ and $\gamma_{n+1}$ agree. Because 


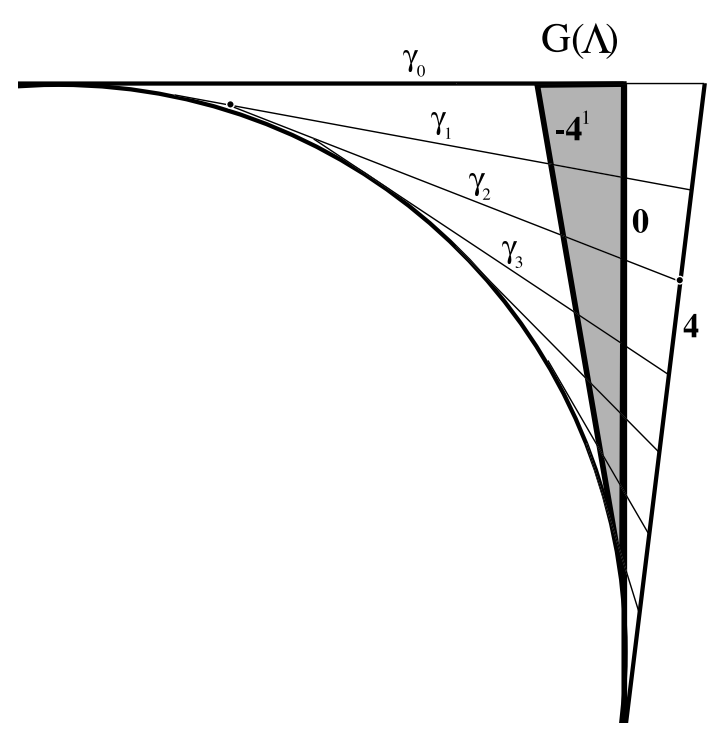

Figure 5: The geometry of lemma[5] the regular components $\gamma_{n}$ intersect transversally the boundaries $-4^{1}$ and $\mathbf{0}$ of the exit domain $G(\Lambda)$, creating pseudo-hyperbolic points.

$n<N-1$, the point $T_{n+1}$ lies outside $G(\Lambda)$, which completes the induction. An exception may occur for $n=N-1$, in the non-generic case in which $T_{N-1}$ lies on the boundary segment $-4^{2}$ of $G(\Lambda)$. In this case $\gamma_{N-1}$ is tangent to that boundary.

From the above lemma, we see that the segments $\gamma_{n}$ decompose $G(\Lambda)$ into $N-1$ quadrilaterals, plus a small residual region near the tip. We are then led to consider the half-open quadrilaterals $\Lambda_{n}^{\text {in }} \subset \Lambda$, given by

$$
\begin{aligned}
\Lambda_{n}^{\text {in }} & =\left\langle\gamma_{0}, G\left(\gamma_{n-1}\right), \gamma_{1}, G\left(\gamma_{n}\right)\right\rangle \\
& =\left\langle\mathbf{1},(-\mathbf{4 n}+\mathbf{4})^{1}, \mathbf{5}^{1},(-\mathbf{4 n})^{1}\right\rangle \quad n=1, \ldots, N-1 .
\end{aligned}
$$

The sides are oriented in such a way that

$$
\gamma_{0}, G\left(\gamma_{n-1}\right) \cap \Lambda_{n}^{\text {in }}=\emptyset \quad \gamma_{1}, G\left(\gamma_{n}\right) \cap \Lambda_{n}^{\text {in }} \neq \emptyset .
$$

We also represent polygons as lists of vertices

$$
\Lambda_{n}^{\text {in }}=\left[Q_{0}(n), Q_{0}(n-1), Q_{1}(n-1), Q_{1}(n)\right] \quad Q_{0,1}(n)=\gamma_{0,1} \cap G\left(\gamma_{n}\right) .
$$

Below, we will show that the sets $\Lambda_{n}^{\text {in }}$ are atoms of $L^{\text {in }}$. Since these domains are bounded by regular components, we shall call them regular atoms; all other atoms will be termed irregular.

From (24), we see that the vertices of $\Lambda_{n}^{\text {in }}$ are pseudo-hyperbolic points of the map $F$. Indeed, for $n=1, \ldots, N-1$, the point $Q_{0}(n)$ is the intersection of $\gamma_{0}=G(\gamma)$ and a segment in the discontinuity set, and hence it is the left end-point of a pseudo-hyperbolic sequence (see remarks at the end of section 2). The point $Q_{1}(n-1)=F^{4}\left(Q_{0}(n)\right.$ belongs to the same orbit, and since it is the transversal intersection of two segments (by virtue of lemma 
[5), it also is pseudo-hyperbolic. Under the action of $F^{4}$, these points propagate across the sector $\Sigma$, forming a regular pattern of intersecting pseudo-separatrices - see figure 2 , The point $G\left(Q_{0}(n)\right)$, which belongs to $\gamma$, is the right endpoint of the pseudo-hyperbolic sequence that starts at $Q_{0}(n)$.

Theorem 6 The regular atoms of the map $L^{\text {in }}$ are the half-open quadrilaterals (22), with the boundary specified in (23). For each $n$, the atom $\Lambda_{n}^{\text {in }}$ is invariant under $L^{\text {in }}$, with return symbolic dynamics $\left(1^{4(n-1)}\right)$ (the exponent denotes repetition of symbols). The fixed set of $L_{n}^{\text {in }}$ is the segment connecting the vertex $Q_{1}(n)$ to the opposite vertex $Q_{0}(n-1)$, where $Q_{0,1}$ is defined in (24). There are at most five other atoms, of total area $O\left(\lambda^{3}\right)$.

Proof. Consider the quadrilateral

$$
G\left(\Lambda_{n}^{\text {in }}\right)=\left\langle\gamma_{n-1}, G\left(\gamma_{0}\right), \gamma_{n}, G\left(\gamma_{1}\right)\right\rangle .
$$

From the proof of lemma 5 , we know that $\gamma_{n}$ and $G\left(\gamma_{1}\right)$ are glued to $G\left(\Lambda_{n}^{\text {in }}\right)$, but the other two sides are not. Applying $F^{-4(n-1)}$ to each segment in $G\left(\Lambda_{n}^{\text {in }}\right)$ we obtain the quadrilateral $\left\langle\gamma_{0}, G\left(\gamma_{n-1}\right), \gamma_{1}, G\left(\gamma_{n}\right)\right\rangle$, and since the iterates of these segments remain within $\Sigma$, we deduce from (22) that $\Lambda_{n}^{\text {in }}$ contains an atom of $L^{\text {in }}$, glued to $\gamma_{1}$ and $G\left(\gamma_{n}\right)$, with transit time $4(n-1)$.

It remains to show that $\Lambda_{n}^{\text {in }}$ is actually an atom. The case $n=1$ is trivial, since $\Lambda_{1}^{\text {in }}=\Lambda \cap G(\Lambda)$, with zero transit time. For $n>1$, we must verify that, in the recursive construction of $\Lambda_{n}^{\text {in }}$, no forward image of the segment $\gamma_{n-1}^{+}=\gamma_{n-1} \cap \Xi$ (the piece of $\gamma_{n-1}$ that gets cut off by the discontinuity) reaches $G\left(\Lambda_{n}^{\text {in }}\right)$ before $\gamma_{n}$, namely that

$$
\bigcup_{t=1}^{4} F^{t}\left(\gamma_{n-1}^{+}\right) \cap G\left(\Lambda_{n}^{\mathrm{in}}\right)=\emptyset \quad n>1
$$

Let $z \in \gamma_{n-1}^{+}$. From lemma 5 and table 1, we have $z \in G\left(\Omega_{0}\right)$. We have two cases. If $F(z) \in \Omega_{0}$, then $F^{2}(z) \in G\left(\Omega_{0}\right)$, and $F^{3}(z)$ is either again in $\Omega_{0}$, or in $\langle-\mathbf{1}, \mathbf{2}, \mathbf{3}\rangle$; in either case $F^{4}(z)$ is outside $\Sigma$, hence outside $G\left(\Lambda_{n}^{\text {in }}\right)$. If $F(z) \in \Omega_{1}$, then $F(z) \in\langle\mathbf{- 1 , 2 , 3}\rangle$, hence $F^{2}(z) \in\langle\mathbf{0}, \mathbf{3}, \mathbf{4}\rangle$. The points of the latter triangle map either to $G\left(\Omega_{0}\right)$ or to $\Lambda$. From neither set it is possible to reach $G\left(\Lambda_{n}^{\text {in }}\right)$ in one iteration. We have established equation (25), and $\Lambda_{n}^{\text {in }}$ is an atom of $L^{\text {in }}$.

Generically, the point $T_{N}$ lies between $G\left(T_{0}\right)$ and $G\left(T_{1}\right)$, and the residual region consists of one quadrilateral, two triangles and two triangular sectors. At isolated parameter values (determined by the condition that $1 / 4(1-4 \rho)$ be an integer), we have $T_{N}=G\left(T_{0}\right)$, and the residual region reduces to one triangular sector, while the regular atom $\Lambda_{N}$ degenerates to a triangle. By construction, the total area of the residual region is $O\left(\lambda^{3}\right)$ in both cases.

From (23), we see that $\gamma_{1}$ and $G\left(\gamma_{n}\right)$ are part of the included boundary of $\Lambda_{n}^{\text {in }}$, and since $\gamma_{n}=F^{4(n-1)}\left(\gamma_{1}\right)$, the transit time of this atom is equal to $4(n-1)$, which is even. With 
the notation of lemma 4, we have $s=2(n-1)$, and hence Fix $L_{n}^{\text {in }}=F^{2(1-n)}($ Fix $G) \cap \Lambda_{n}^{\text {in }}$. Now, by symmetry,

$$
\gamma_{n} \cap G\left(\gamma_{n}\right) \in \operatorname{Fix} G \cap \Sigma \quad n=0, \ldots,\lfloor(N-1) / 2\rfloor .
$$

We also have the symmetric points

$$
F^{4 n-2}\left(\gamma_{0}\right) \cap G \circ F^{4 n-2}\left(\gamma_{0}\right) \in \operatorname{Fix} G \quad n=1, \ldots,\lfloor(N-1) / 2\rfloor
$$

which lie in the South-West sector. (With a slight abuse of notation, we have denoted by $F^{4 n-2}\left(\gamma_{0}\right)$ the regular component of that set, with respect to $\mathcal{E}$.) Applying the map $F^{-2}$ to the points (27), we obtain a second sequence of points in $\Sigma$

$$
\gamma_{n-1} \cap G\left(\gamma_{n}\right) \in F^{-2}(\operatorname{Fix} G) \cap \Sigma \quad n=1, \ldots,\lfloor(N-1) / 2\rfloor .
$$

Then, by applying repeatedly the identity

$$
F^{-4}\left(\gamma_{j} \cap G\left(\gamma_{k}\right)\right)=\gamma_{j-1} \cap G\left(\gamma_{k+1}\right)
$$

to pairs of adjacent points in the above two sequences, we translate images of Fix $G$ within $\Lambda$, and we see that Fix $L_{n}^{\text {in }}$ is the segment connecting $\gamma_{0} \cap G\left(\gamma_{n-1}\right)$ to $\gamma_{1} \cap G\left(\gamma_{n}\right)$. Comparison with (22) shows that this is the segment connecting the North-East and South-West corners of the $n$th atom (with the obvious modification if the last regular atom degenerates into a triangle).

Now, $L^{\text {in }}$ is an involution (lemma 3), and its regular atom $\Lambda_{n}^{\text {in }}$ is maximal, being bound by images of the discontinuity line. Then every regular atom is invariant, because it contains an invariant segment (lemma 2).

\subsection{The sector map of the 2-cycle}

The dynamics of the map $L^{\text {out }}$ is dominated by the 2-cycle of $F$ given in table (9), which plays a role analogous to the 1-cycle for the map $L^{\text {in }}$. The 2-cycle generates the regular atoms of the map. There are also irregular atoms, but they have negligible measure.

We noted that the 2-cycle is symmetrical, and it belongs to Fix $H$ (see beginning of section 3 ). We consider the element of the cycle that lies within the atom $\Omega_{1}$ of $F$, denoting by $\mathcal{E}^{\prime}$ the ellipse bounding its cell (figure (4). From the data (9), we find that the segment $\gamma=\mathbf{0}$ is tangent to $\mathcal{E}^{\prime}$ at the point $(0,1 / 2)$. By $H$-symmetry, the segment $H(\gamma)=H(\mathbf{0})=$ 2 - see (13) - is also tangent to $\mathcal{E}^{\prime}$ at the point $T_{0}^{\prime}=H(0,1 / 2)=(\lambda / 2,1 / 2)$. The open region delimited by the segments $\mathbf{0}$ and $\mathbf{2}$, and the elliptical arc connecting $T_{0}^{\prime}$ to $H\left(T_{0}^{\prime}\right)$ clockwise will be called the 2-sector $\Sigma^{\prime}$

$$
\Sigma^{\prime}=\left\langle\mathcal{E}^{\prime}, \mathbf{2}, \mathbf{0}\right\rangle
$$

This region is $H$-invariant, and both $\mathbf{0}$ and $\mathbf{2}$ are are glued to it. 
The analysis of the dynamics of the 2-sector now proceed as for the 1-sector; we'll use the same notation, with primed symbols. First we define entry and exit domains - the analogue of (10)

$$
\Lambda^{\prime}=\Sigma^{\prime} \backslash F^{4}\left(\Sigma^{\prime}\right) \quad \Xi^{\prime}=F^{4}\left(\Sigma^{\prime}\right) \backslash \Sigma^{\prime}=F^{4} \circ H\left(\Lambda^{\prime}\right) .
$$

Let

$$
M=\left\lfloor\frac{1}{2(1-4 \rho)}-\frac{1}{2}\right\rfloor \sim\left\lfloor\frac{\pi}{2 \lambda}\right\rfloor \quad \text { as } \quad \lambda \rightarrow 0
$$

where $\rho$ is the rotation number; this is the analogue of equation (21). For $m=0, \ldots, M-1$, we let $\gamma_{m}^{\prime}=(\mathbf{4} \mathbf{m}+\mathbf{2})^{2}$ be the regular component of $F^{4 m}(\mathbf{2})$ with respect to $\mathcal{E}^{\prime}$, denoting by $T_{m}^{\prime}$ the corresponding point of tangency. The segments $\gamma_{m}^{\prime}$ are pairwise quasi-parallel, and their orientations agree. The following result is the twin of lemma 1 .

Lemma 7 The restriction to the closure of the sector $\Sigma^{\prime}$ of the maps $F^{ \pm 4}$ is continuous. The symbolic dynamics is $(1,0,1,0)$ for points in $\Sigma^{\prime} \backslash H\left(\Lambda^{\prime}\right)$ and $(1,0,1,1)$ for points in $H\left(\Lambda^{\prime}\right)$.

Proof. From (28) we find that $F\left(\Sigma^{\prime}\right)=\left\langle\mathcal{E}^{\prime}, \mathbf{3}, \mathbf{1}\right\rangle$. Considering that $F\left(T_{0}^{\prime}\right)=((1+$ $\left.\left.\lambda^{2}\right) / 2, \lambda / 2\right) \in \Omega_{0}$, from table 1 we deduce that $F\left(\Sigma^{\prime}\right) \subset \Omega_{0}$. Then we have $F^{2}\left(\Sigma^{\prime}\right) \subset G\left(\Omega_{0}\right)$, hence $F^{3}\left(\Sigma^{\prime}\right) \cap \gamma=\emptyset$, and so $F^{4}$ is continuous on $\Sigma^{\prime}$. By time-reversal symmetry, the inverse $F^{-4}$ is also continuous on $\Sigma^{\prime}$. Since the boundaries of $\Sigma^{\prime}$ are glued to it, and remain glued under iteration, continuity extends to the boundary.

Every point in $\Sigma^{\prime} \backslash H\left(\Lambda^{\prime}\right)$ follows the 2-cycle, and so the return dynamics is $(1,0,1,0)$. We determine the itinerary of $H\left(\Lambda^{\prime}\right)$. We have $\Lambda^{\prime}=\left\langle\mathbf{0}, \mathbf{6}^{2}, \mathbf{2}\right\rangle$, and hence $H\left(\Lambda^{\prime}\right)=\left\langle\mathbf{2}, \mathbf{0},-\mathbf{4}^{2}\right\rangle \in$ $\Omega_{1}$. This gives $F \circ H\left(\Lambda^{\prime}\right)=\langle\mathbf{3}, \mathbf{1},-\mathbf{3}\rangle \subset \Omega_{0}$, whence $F^{2} \circ H\left(\Lambda^{\prime}\right) \subset \Omega_{1}$, and finally $F^{3} \circ H\left(\Lambda^{\prime}\right)=\left\langle\mathbf{5}^{2}, \mathbf{3}, \mathbf{- 1}\right\rangle \subset \Omega_{1}$. All inclusion relations may be verified with the aid of table 1, together with (12) and (13).

The continuity of $F^{4}$ on $\Sigma^{\prime}$ implies the existence of an orderly array of regular components.

Lemma 8 For $m=1, \ldots, M-1$, the regular component $\gamma_{m}^{\prime}$ intersects $\gamma$ transversally, apart from a possible non-generic degeneracy of $\gamma_{M-1}^{\prime} \cap H\left(\gamma_{1}^{\prime}\right)$ into a segment. For $m>1$, $\gamma_{m}^{\prime}$ connects $\gamma_{m-1}^{\prime}$ to 4 .

Proof. The proof of this statement is analogous to that of lemma 5 , with the added simplification that continuity now extends to the boundary. The same applies to the argument concerning the degeneracy for $\gamma_{M-1}^{\prime}$. We omit the details for the sake of brevity.

The following result is the analogue of theorem 6 for the 2 -sector $\Sigma^{\prime}$. 
Lemma 9 For $m=1, \ldots, M-1$, the regular atoms $\Lambda_{m}^{\prime}$ of the transit map $\Lambda^{\prime} \rightarrow H\left(\Lambda^{\prime}\right)$ induced by $F$ are the half-open quadrilaterals

$$
\Lambda_{m}^{\prime}=\left\langle\gamma_{0}^{\prime}, H\left(\gamma_{m-1}^{\prime}\right), \gamma_{1}^{\prime}, H\left(\gamma_{m}^{\prime}\right)\right\rangle=\left\langle\mathbf{2},(-\mathbf{4 m}+\mathbf{4})^{2}, \mathbf{6}^{2},(-\mathbf{4 m})^{2}\right\rangle
$$

The boundary sides $\gamma_{0}^{\prime}$ and $H\left(\gamma_{m}^{\prime}\right)$ belong to $\Lambda_{m}^{\prime}$, the other don't. The return symbolic dynamics of the $m$-th atom is $\left((1,0)^{2 m-2}\right)$. There are at most five other atoms, of total area $O\left(\lambda^{3}\right)$.

Proof. The proof is analogous to that of theorem 6, with $H$ in place of $G$, and primed variables. Identity (25) now reads

$$
\bigcup_{t=1}^{4} F^{t}\left(\gamma_{m-1} \cap X i^{\prime}\right) \cap \Lambda_{m}^{\prime}=\emptyset \quad m>1 .
$$

To establish this identity, we note that $\gamma_{m-1}^{\prime}$ intersects $\gamma$. The right component $\gamma_{m-1}^{\prime} \cap \Sigma^{\prime}$ will iterate to produce $\gamma_{m}^{\prime}$, and we must ensure that the left component $\gamma_{m-1}^{\prime} \cap \Xi^{\prime}$ does not interfere with this process. We find

$$
\Xi_{1}^{\prime} \stackrel{\text { def }}{=} \Xi^{\prime} \cap \Omega_{1} \subset F^{-1}(\Lambda)=\langle-\mathbf{1}, \mathbf{4}, \mathbf{0}\rangle
$$

and the map $F^{4}$ is continuous over the closure of that domain, from lemma 1, apart from a sliding singularity on $\mathbf{- 1}$ and $\mathbf{0}$. Thus

$$
\begin{array}{ll}
F^{1}\left(\Xi_{1}^{\prime}\right) \subset\left\langle\mathbf{0}, \mathbf{5}^{1}, \mathbf{1}\right\rangle & F^{2}\left(\Xi_{1}^{\prime}\right) \subset\left\langle\mathbf{1}, \mathbf{6}^{1}, \mathbf{4}\right\rangle \\
F^{3}\left(\Xi_{1}^{\prime}\right) \subset\left\langle\mathbf{2}, \mathbf{7}^{1}, \mathbf{3}\right\rangle & F^{4}\left(\Xi_{1}^{\prime}\right) \subset\left\langle\mathbf{3}, \mathbf{8}^{1}, \mathbf{4}\right\rangle
\end{array}
$$

all of which lie outside $\Xi^{\prime}$, from table 1, Similarly, we find

$$
\Xi_{0}^{\prime} \stackrel{\text { def }}{=} \Xi^{\prime} \cap \Omega_{0} \subset\langle-\mathbf{1}, \mathbf{0}, \mathbf{3}\rangle
$$

and one verifies that $F^{3}$ is continuous on the closure of that set. Hence

$$
F^{1}\left(\Xi_{0}\right) \subset\langle\mathbf{0}, \mathbf{1}, \mathbf{4}\rangle \quad F^{2}\left(\Xi_{0}\right) \subset\left\langle\mathbf{1}, \mathbf{2}, \mathbf{5}^{2}\right\rangle \quad F^{3}\left(\Xi_{0}\right) \subset\left\langle\mathbf{2}, \mathbf{3}, \mathbf{6}^{2}\right\rangle .
$$

None of these sets intersects $\Xi^{\prime}$; the last one is adjacent to $\Xi^{\prime}$ an intersects $\gamma$. Thus $F^{4}\left(\Xi_{0}\right)$ is contained in the union of two triangles, which clearly lie outside $\Xi^{\prime}$.

As for the sector $\Sigma$, the vertices of the atoms $\Lambda_{m}^{\prime}$ of the transit map over $\Sigma^{\prime}$ are pseudohyperbolic points of the map $F$. Indeed such vertices result from transversal intersections of regular components - see equation (30) and figure 4. Two vertices of the atom $\Lambda_{1}^{\prime}$ belong to $\mathbf{0}=\gamma$, and hence they are right end-points of pseudo-hyperbolic sequences.

\subsection{Structure of $L^{\text {out }}$}

To construct the atoms of the map $L^{\text {out}}$, we need to connect dynamically the exit domain of the sector $\Sigma$ to the entry domain of the sector $\Sigma^{\prime}$, and vice-versa. These connections will 
not match perfectly, but the mismatch will involve sets of negligible measure. In addition, there will be one atom of $L^{\text {out }}$ by-passing $\Sigma^{\prime}$ altogether (see figure 4).

All points leaving $\Sigma$ via $\Lambda^{\text {out }}$ end up in $\Xi$, and since we want to identify the points that will enter $\Sigma^{\prime}$, we examine inverse images of the entry domain $\Lambda^{\prime}$. The second pre-image of $\Lambda^{\prime}$ intersects $\Xi$, and so in order to connect the 1-sector to the 2-sector, we consider the intersection and symmetric difference of the sets $\Xi$ and $F^{-2}\left(\Lambda^{\prime}\right)$. Likewise, connecting back the 2-sector to the 1-sector will involve the sets $\Xi^{\prime}$ and $F^{-1}(\Lambda)$.

Our first lemma quantifies these intersections and symmetric differences. Its proof will also provide information about the atoms of $L^{\text {out }}$.

Lemma 10 The points of $\Lambda^{\text {out }}$ return to $\Sigma$ either via the domain $\Upsilon_{1}=\left\langle-\mathbf{2}, \mathbf{5}^{1}, \mathbf{4}\right\rangle$, or via $\Lambda^{\prime}$, apart from a set of points of measure $O\left(\lambda^{3}\right)$.

Proof. First, we connect $\Xi$ to $\Lambda^{\prime}$. By the continuity of $F^{-2}$ on $\Sigma^{\prime}$ (lemma 7), we have $F^{-2}\left(\Lambda^{\prime}\right)=\langle-\mathbf{2}, \mathbf{0}, \mathbf{4}\rangle$. Defining

$$
\Upsilon_{1}=\left\langle-\mathbf{2}, \mathbf{5}^{1}, \mathbf{4}\right\rangle \quad \Upsilon_{2}=\left\langle-\mathbf{2}, \mathbf{4}, \mathbf{0}, \mathbf{5}^{1}\right\rangle
$$

one verifies that

$$
\Xi \Delta F^{-2}\left(\Lambda^{\prime}\right)=\Upsilon_{1} \cup\left\langle-\mathbf{2}, \mathbf{5}^{1}, \mathbf{0}\right\rangle \quad \Xi \cap F^{-2}\left(\Lambda^{\prime}\right)=\Upsilon_{2} .
$$

From the orthogonality relations $-\mathbf{2}\left\|\mathbf{0} \perp \mathbf{5}^{1}\right\| \mathbf{1}$, and the fact that the length of the side $\mathbf{0}$ of $\left\langle\mathbf{- 2}, \mathbf{5}^{1}, \mathbf{0}\right\rangle$ is $O(\lambda)$, we conclude that $\left|\left\langle-\mathbf{2}, \mathbf{5}^{1}, \mathbf{0}\right\rangle\right|=O\left(\lambda^{3}\right)$, where $|\cdot|$ denotes the two-dimensional area determined by the metric $\mathcal{Q}$. Thus

$$
\left|\Xi \Delta F^{-2}\left(\Lambda^{\prime}\right)\right|=\left|\Upsilon_{1}\right|+O\left(\lambda^{3}\right) .
$$

We must now consider the dynamics of $\Upsilon_{1}$ and of $\Upsilon_{2}$, from $\Xi$ to $F^{-1}(\Lambda)$. We begin with the former. From (16) and (32), we find, using continuity

$$
F^{2}\left(\Upsilon_{1}\right)=\left\langle\mathbf{0}, \mathbf{7}^{+}, \mathbf{6}^{2}\right\rangle \quad F^{-1}(\Lambda)=\langle-\mathbf{1}, \mathbf{0}, \mathbf{4}\rangle
$$

where $\mathbf{7}^{+}=F^{2}\left(\mathbf{5}^{1} \cap \Xi\right)$. Hence $F^{2}\left(\Upsilon_{1}\right) \cap F^{-1}(\Lambda) \neq \emptyset$, and so some points in $\Upsilon_{1}$ reach $\Lambda$ in 3 iterations. However, not all of them do. To see this, we note that $\mathbf{5}^{1}$ intersects Fix $H$ transversally, and this intersection belongs to the boundary of $\Upsilon_{1}$. Then, by symmetry,

$$
\Upsilon_{1} \Delta H\left(\Upsilon_{1}\right)=\Upsilon_{1}^{\prime} \cup H\left(\Upsilon_{1}^{\prime}\right) \quad \text { where } \quad \Upsilon_{1}^{\prime}=\left\langle-\mathbf{2}, \mathbf{5}^{1},-\mathbf{3}\right\rangle
$$

The $H$-symmetric set (34) will be called a turnstile (figure 6); by construction, $\left|\Upsilon_{1}^{\prime}\right|=$ $\left|H\left(\Upsilon_{1}^{\prime}\right)\right|$. Then the set $F^{2}\left(\Upsilon_{1}^{\prime} \cup H\left(\Upsilon_{1}^{\prime}\right)\right)$ is also a turnstile, which lies on the boundary of $F^{-1}(\Lambda)$. Finally,

$$
\Upsilon_{1}^{\prime} \subset \Upsilon_{1} \quad F^{2}\left(\Upsilon_{1}^{\prime}\right) \cap F^{-1}(\Lambda)=\emptyset
$$



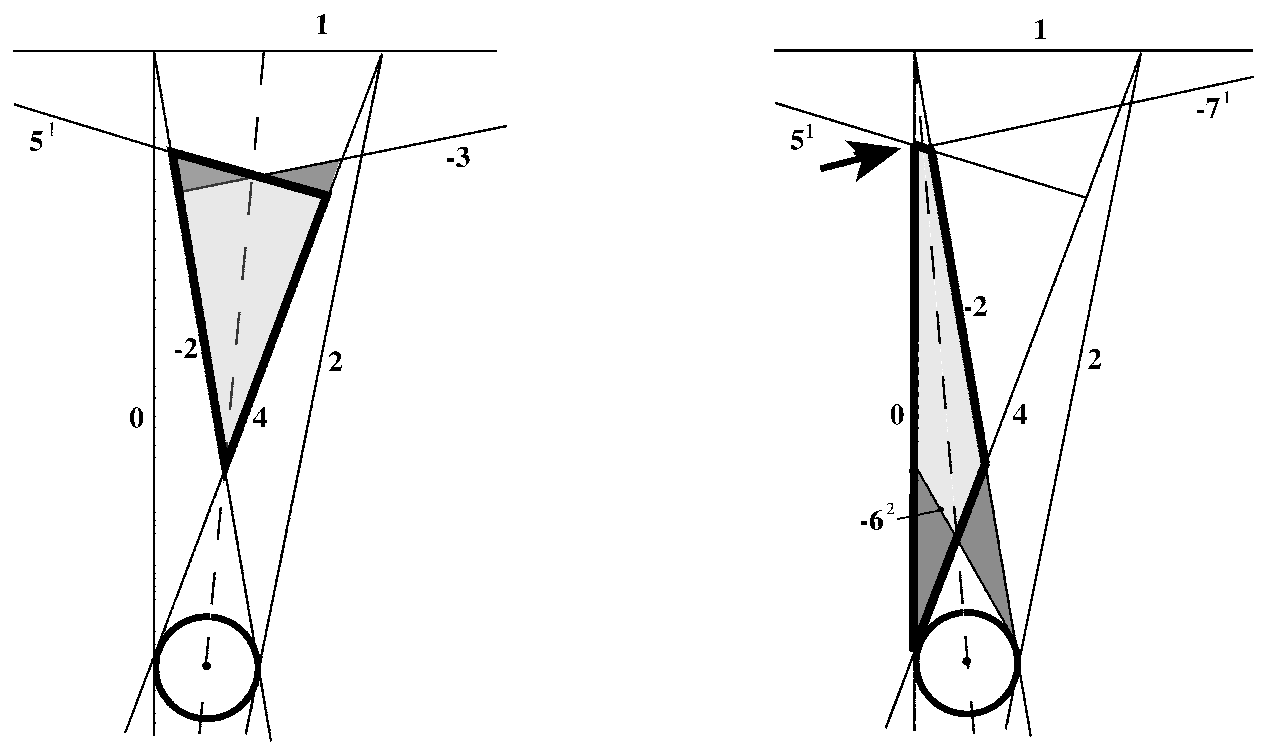

Figure 6: Left: Symmetrisation of the set $\Upsilon_{1}$ (the thick-sided triangle), with respect to the symmetry line Fix $H$ (the dashed line). The two darker triangles represent the turnstile. Right: Symmetrisation of the quadrilateral $\Upsilon_{2}$, with the appearance of two turnstiles; the arrow denotes the location of the smaller turnstile. The dashed line is Fix $F^{-4} \circ H$. The symmetrised polygons (light grey) are isometric images of the first and second regular atom of $L^{\text {out }}$, respectively.

which identifies $\Upsilon_{1}^{\prime}$ as the set of points of $\Lambda$ that map into $\Upsilon_{1}$, but do not return to $\Lambda$ with it. From the relations $\mathbf{- 3} \| \mathbf{5}^{1}$ and the fact that the length of $\bar{\Upsilon}_{1} \cap \mathbf{5}^{1}$ is $O(\lambda)$, we have $\left|\Upsilon_{1}^{\prime}\right|=O\left(\lambda^{3}\right)$. Thus

$$
\left.\mid F^{3}\left(\Upsilon_{1}\right) \cap \Lambda\right)|=| \Upsilon_{1} \mid+O\left(\lambda^{3}\right)
$$

There is an analogous phenomenon in the dynamics of $\Upsilon_{2}$, defined in equation (31). Since $H(\mathbf{0})=\mathbf{2}$, we have $F^{-4} \circ H(\mathbf{0})=-\mathbf{2}$, and $F^{-4} \circ H$ is an involution. The boundary of $\Upsilon_{2}$ intersects Fix $\left(F^{-4} \circ H\right)$ in two points, generating two turnstiles (figure 6, right). We are interested in the intersection $5^{1} \cap \operatorname{Fix}\left(F^{-4} \circ H\right)$, which corresponds to the turnstile

$$
\Upsilon_{2}^{\prime} \cup F^{-4} \circ H\left(\Upsilon_{2}^{\prime}\right) \quad \text { where } \quad \Upsilon_{2}^{\prime}=\left\langle\mathbf{0}, \mathbf{5}^{1},-\mathbf{7}\right\rangle .
$$

Then the set $F^{6}\left(\Upsilon_{2}^{\prime} \cup F^{-4} \circ H\left(\Upsilon_{2}^{\prime}\right)\right)$ is also a turnstile, which lies on the boundary of $F^{-1}(\Lambda)$. Finally,

$$
\Upsilon_{2}^{\prime} \subset \Upsilon_{2} \quad F^{6}\left(\Upsilon_{2}^{\prime}\right) \cap F^{-1}(\Lambda)=\emptyset
$$

which identifies $\Upsilon_{2}^{\prime}$ as the set of points of $\Lambda$ that map into $\Upsilon_{2}$, hence to $\Lambda^{\prime}$, but do not return to $\Lambda$ with it. From the relation $\mathbf{5}^{1} \| \mathbf{- 7}$, and the fact that the length of $\bar{\Upsilon}_{2} \cap \mathbf{5}^{1}$ is $O\left(\lambda^{2}\right)$, we have $\left|\Upsilon_{2}^{\prime}\right|=O\left(\lambda^{4}\right)$. This estimate, together with equations (32), and the estimates (33) and (35), give the desired result.

We now identify the regular atoms of $L^{\text {out }}$. Let $\Upsilon_{1,2}$ be as in (31), and let $\Lambda_{m}^{\prime}$ be as in 
lemma 9, We define the sets

$$
\begin{aligned}
& \Lambda_{1}^{\text {out }}=G \circ F^{-4}\left(\Upsilon_{1} \cap H\left(\Upsilon_{1}\right)\right)=\langle\mathbf{0}, \mathbf{8}, \mathbf{7}, \mathbf{1}\rangle \cap \Lambda \\
& \Lambda_{2}^{\text {out }}=G \circ F^{-4}\left(\Upsilon_{2} \cap F^{4} \circ H\left(\Upsilon_{2}\right)\right)=\langle\mathbf{0}, \mathbf{1 2}, \mathbf{5}, \mathbf{1 1}, \mathbf{1}, \mathbf{7}\rangle \cap \Lambda \\
& \Lambda_{m}^{\text {out }}=G \circ F^{-6}\left(\Lambda_{m-1}^{\prime}\right)=\langle\mathbf{1}, \mathbf{4 m}-\mathbf{1}, \mathbf{5}, \mathbf{4 m}+\mathbf{3}\rangle \cap \Lambda \quad 3 \leq m \leq M .
\end{aligned}
$$

The rightmost equalities mean that the polygon on the left can be assembled with an appropriate choice of the components listed on the right, in the given order. The issue of uniqueness of a polygon with such a labelling is unimportant, as these polygons are continuous images of well-defined objects. The boundaries of the sets $\Lambda_{m}^{\text {out }}$ are specified by the following non-empty intersections

$$
\mathbf{5}, \mathbf{4} \mathbf{m}-\mathbf{1} \cap \Lambda_{m}^{\text {out }} \neq \emptyset \quad m>1
$$

and no other. In particular, $\Lambda_{1}^{\text {out }}$ is open. The sets (37) are indeed quadrilaterals, apart from $\Lambda_{2}^{\text {out }}$, which is a hexagon.

All atoms are tangent to $\mathbf{1}$, and we let $P_{0}(m)$ be the left end-point of the intersection of $\Lambda_{m}^{\text {out }}$ with 1 . Then we let $P_{1}(m-1)$ be the vertex of $\Lambda_{m}^{\text {out }}$ which is opposite to $P_{0}(m)$ (the term 'opposite' refers to the fact that all atoms have an even number of sides). We find that $P_{1}(0)$ and $P_{1}(1)$ are, respectively, the lower end-point of the intersection of $\Lambda_{1}^{\text {out }}$ and $\Lambda_{2}^{\text {out }}$ with $\mathbf{0}$; likewise, for $m \geq 2$, the point $P_{1}(m)$ is the left end-point of the intersection of $\Lambda_{m}^{\text {out }}$ with $\mathbf{5}^{1}$. With these prescription we can characterize the sets $\Lambda_{m}^{\text {out }}$ in terms of the vertices $P_{0,1}$, as follows

$$
\Lambda_{m}^{\text {out }}=\left[P_{0}(m), P_{0}(m-1), P_{1}(m-1), P_{1}(m)\right] \quad m>2 .
$$

A complete description for the cases $m=1,2$ it not needed at this stage, and will be given in section 4.1 .

We can now state the main result of this section.

Theorem 11 The regular atoms $\Lambda_{m}^{\text {out }}$ of the map $L^{\text {out }}$ are the polygons (37), with the boundary specified in (38). For each $m$, the atom $\Lambda_{m}^{\text {out }}$ is invariant under $L^{\text {out }}$, and its return symbolic dynamics is

$$
\left(1,1,1,(0,1)^{2 m-1}, 1,1\right) .
$$

The fixed set of $L_{m}^{\text {out }}$ is a segment connecting $P_{0}(m)$ to the opposite vertex $P_{1}(m-1)$. In addition, there is a (possibly infinite) set of irregular atoms of total area $O\left(\lambda^{3}\right)$.

Proof. From lemma 10, it suffices to consider the pre-images of $\Upsilon_{1}$ and $\Lambda^{\prime}$, and from the rightmost equation in (32), we may replace $\Lambda^{\prime}$ by $\Upsilon_{2}$.

From the proof of lemma [10, we see that the part of $\Upsilon_{1}$ that maps to $\Lambda$ under $F^{3}$ is the $H$-symmetrical quadrilateral

$$
\Upsilon_{1} \cap H\left(\Upsilon_{1}\right)=\left\langle-\mathbf{3}, \mathbf{5}^{1}, \mathbf{4},-\mathbf{2}\right\rangle \subset \Xi .
$$


To obtain the first atom $L_{1}^{\text {out }}$, we apply $G \circ F^{-4}$ to it, which maps it back to $\Lambda$. We obtain

$$
\Lambda_{1}^{\text {out }} \in G(\langle-\mathbf{7}, \mathbf{1}, \mathbf{0},-\mathbf{6}\rangle \cap G(\Lambda))=\langle\mathbf{8}, \mathbf{7}, \mathbf{1}, \mathbf{0}\rangle \cap \Lambda,
$$

as desired.

We have $\Upsilon_{2} \subset \Xi$, and so $G \circ F^{-4}\left(\Upsilon_{2}\right) \subset \Lambda$. From the proof of lemma 10, we see that the part of $\Upsilon_{2}$ that maps to $\Lambda$ under $F^{7}$ is the $F^{-4} \circ H$-symmetrical hexagon

$$
\Upsilon_{2} \cap F^{-4} \circ H\left(\Upsilon_{2}\right)=\left\langle\mathbf{0},-\mathbf{7}^{1}, \mathbf{5}^{1}, \mathbf{- 2}, \mathbf{4},-\mathbf{6}^{2}\right\rangle \subset\left\langle\mathbf{0},-\mathbf{2}, \mathbf{4},-\mathbf{6}^{2}\right\rangle=F^{-2}\left(\Lambda_{1}^{\prime}\right)
$$

where $\Lambda_{1}^{\prime}$ is the first atom of the 2-sector map (lemma 9). The atom $\Lambda_{2}^{\text {out }}$ of $L^{\text {out }}$ is the image under $G \circ F^{-4}$ of the symmetrised hexagon in (42). We obtain

$$
\Lambda_{2}^{\text {out }}=\langle\mathbf{0}, \mathbf{1 2}, \mathbf{5}, \mathbf{1 1}, \mathbf{1}, \mathbf{7}\rangle \cap \Lambda \text {. }
$$

The rest of $\Upsilon_{2}$, namely the triangle

$$
F^{-2}\left(\Lambda^{\prime} \backslash \Lambda_{1}^{\prime}\right)=\left\langle\mathbf{0},-\mathbf{6}^{2}, \mathbf{4}\right\rangle \subset \Xi
$$

is covered, apart from a set of measure $O\left(\lambda^{3}\right)$, by the second pre-image of the atoms $\Lambda_{m}^{\prime}, m=2, \ldots, M-1$ of the 2-sector map, from lemma 9. The above inclusion, together with lemmas 1 and 7 , show that $F^{-6}$ is continuous on $\Lambda^{\prime} \backslash \Lambda_{1}^{\prime}$. Then, from lemma 9, we have

$$
G \circ F^{-6}\left(\Lambda_{m-1}^{\prime}\right)=\langle\mathbf{1}, \mathbf{4 m}-\mathbf{1}, \mathbf{5}, \mathbf{4} \mathbf{m}+\mathbf{3}\rangle \cap \Lambda
$$

as desired

Verifying that the polygons defined in (37) have the boundaries as given in (23) is a simple exercise in orientation, recalling the conventions of table 1.

The code of each point in $\Lambda$ begins with the string $(1,1,1,0)$, associated with the map $\Lambda^{\text {out }} \rightarrow \Xi$ (lemma 1). One verifies directly that the points in $\Lambda_{1}^{\text {out }}$ return to $\Lambda$ with the code $(1,1,1)$. For $m>1$, the $m$-th atom $\Lambda_{m}^{\text {out }}$ follows the itinerary:

2 iterations to reach $\Lambda^{\prime}$, with code $(1,0)$ (lemma 7);

$4(m-1)$ iterations to reach $H\left(\Lambda^{\prime}\right)$, with code $(1,0)^{2 m-2}$ (lemma 9);

4 iterations to reach $\Xi^{\prime}$, with code $(1,0,1,1)$ (lemma 7);

1 iteration to reach $\Lambda$, with code (1).

Putting everything together, we obtain the code (40). In particular, the transit time of the $m$-th atom is odd, and equal to $4 m+3$. From lemma 4 , we then have

$$
\text { Fix } L_{m}^{\text {out }}=F^{2 m+1}(\text { Fix } H) \cap \Lambda_{m}^{\text {out }},
$$

and it remains to show that all these intersections are non-empty.

We identify the fixed set of an involution within each regular atom. The atom $\Lambda_{1}^{\text {out }}$ is the image under $G \circ F^{-4}$ of the set $\Upsilon_{1} \cap H\left(\Upsilon_{1}\right)$, which intersects Fix $H$. Hence $\Lambda_{1}^{\text {out }}$ 
intersects $G \circ F^{-4}($ Fix $H)=F^{3}($ Fix $H)$, in accordance with (44). Likewise, the atom $\Lambda_{2}^{\text {out }}$ is the image under $G \circ F^{-4}$ of the set $\Upsilon_{2} \cap F^{-4} \circ H\left(\Upsilon_{2}\right)$ which intersects $F^{-2}($ Fix $H)$. Hence $\Lambda_{2}^{\text {out }}$ intersects $G \circ F^{-6}($ Fix $H)=F^{5}($ Fix $H)$, as desired.

The symmetry properties of the atoms $\Lambda_{m}^{\text {out }}$ for $m \geq 3$ are proved with an argument analogous to that used in the proof of theorem 6. Specifically, in place of (26) and (27), we have the symmetric points

$$
\gamma_{m}^{\prime} \cap H\left(\gamma_{m}^{\prime}\right) \in \operatorname{Fix} H \cap \Sigma^{\prime} \quad m=0, \ldots,\lfloor(M-1) / 2\rfloor
$$

as well as

$$
F^{4 m-2}\left(\gamma_{0}^{\prime}\right) \cap H \circ F^{4 m-2}\left(\gamma_{0}^{\prime}\right) \in \operatorname{Fix} H \quad m=1, \ldots,\lfloor(M-1) / 2\rfloor .
$$

These symmetry lines intersect images of atoms, and by application of $G \circ F^{-t}$, for a suitable $t$, one ensures that the intersection in (44) is non empty also for $m>2$. We omit the details, but we note that, by construction, these sets intersect one vertex of the atom, and hence also the opposite vertex, due to symmetry.

All regular atoms are maximal, being bound by images of the discontinuity line. Then, from lemmas 2 and 3, they are invariant under the map $L^{\text {out }}$.

\section{Quantitative results}

In this section we supplement the geometrical approach to return-map dynamics developed in section 3, with a predominantly algebraic one. To illustrate the technique, we will prove a version of theorem [1]. As we shall see, the proof is conceptually simple, and the details relatively easy to carry out, provided that one is willing to rely heavily on computer assistance to manipulate complicated expressions. For the sake of brevity, the polygons treated in this section do not include their boundaries.

\subsection{Calculation of atom vertices}

The elementary ingredients of our algebraic calculations are the regular atoms of $L^{\text {in }}$ and $L^{\text {out }}$ expressed in terms of their vertices. To obtain explicit expressions for the latter as rational function in $\lambda$, we rely on formulae (22) and (37), which express the polygonal atoms in terms of bounding lines. Our strategy is to express the latter in terms of the cartesian coordinates of their endpoints, then calculate the intersection of neighbouring lines to obtain the corresponding polygonal vertex. All quantities are restricted to $\Omega$, that is, we don't make use of the periodicity of the torus.

Before proceeding to the calculation of vertices, let us introduce the convenient notation

$$
F_{\iota}=F_{\left(\iota_{0} \iota_{1} \cdots \iota_{k-1}\right)}=F_{\left(\iota_{k-1}\right)} \circ F_{\left(\iota_{k-2}\right)} \circ \cdots \circ F_{\left(\iota_{0}\right)}
$$


where $\iota \in\{0,1\}^{k}$ is an (arbitrary) codeword, and

$$
F_{(i)}: \mathbb{R}^{2} \rightarrow \mathbb{R}^{2} \quad\left(\begin{array}{c}
x \\
y
\end{array}\right) \mapsto C \cdot\left(\begin{array}{l}
x \\
y
\end{array}\right)+\left(\begin{array}{l}
i \\
0
\end{array}\right)
$$

where the matrix $C$ was defined in (11). From (2) we see that our piecewise isometry $F$ acts as $F_{(0)}$ (generalized rotation about the origin) on the atom $\Omega_{0}$ and as $F_{(1)}$ (generalized rotation about the fixed point $\left.\left(\frac{1}{2-\lambda}, \frac{1}{2-\lambda}\right)\right)$ on $\Omega_{1}$. It is crucial to distinguish between the expressions $F_{\left(\iota_{0} \iota_{1} \cdots \iota_{k-1}\right)}$, an isometry of the plane, which acts as a generalized rotation about some fixed point, and $F^{k}$, a piecewise isometry of the unit square, acting differently on different domains.

Let us calculate the vertices $P_{0}(m)$ and $P_{1}(m)$ of the regular atoms of $L^{\text {out }}$, see equation (39), where, based on the geometric analysis of section 3 ,

$$
P_{0}(m)=\xi_{m} \cap \mathbf{1}, \quad P_{1}(m)=\xi_{m} \cap \mathbf{5}^{1},
$$

with

$$
\xi_{m}=F_{\left(1^{3}(0,1)^{(2 m-1)} 1^{2}\right)}([(1,0),(1,1)])
$$

We define

$$
\alpha=2 \pi \rho=\cos ^{-1}(\lambda / 2) \quad \theta=\frac{\pi}{2}-\alpha=\sin ^{-1}(\lambda / 2) .
$$

From (11) we see that $\alpha$ is the angle of rotation of the matrix $\mathrm{C}$, while $\theta=2 \pi\left(\frac{1}{4}-\rho\right)$. Hence $\theta$ is the angular departure from the $\lambda=0$ rotation, that is, $4 \theta$ is the (positive) angle between successive regular components of the sector map on $\Sigma$, in the $\mathcal{Q}$-metric.

To evaluate $\xi_{m}$ explicitly for general $m$, we regard the isometry $F_{\left(1^{3}(0,1)^{(2 m-1)} 1^{2}\right)}$ as a rotation by $3 \alpha$ about $z_{1}=\left(\frac{1}{2-\lambda}, \frac{1}{2-\lambda}\right)$, then a rotation by $(4 m-2) \alpha$ about $z_{2}=\left(\frac{2}{4-\lambda^{2}}, \frac{\lambda}{4-\lambda^{2}}\right)$, and finally a rotation by $2 \alpha$ about $z_{1}$. A multiple-angle rotation corresponds to a power of the matrix $C$ and can be executed in a single step, thanks to the formula (a consequence of the Jordan decomposition of $C$ )

$$
C^{k}=\cos (k \alpha)\left(\begin{array}{cc}
1 & 0 \\
0 & 1
\end{array}\right)+\frac{\sin (k \alpha)}{\sqrt{4-\lambda^{2}}}\left(\begin{array}{cc}
\lambda & -2 \\
2 & -\lambda
\end{array}\right) .
$$

Our geometric constructions of the atom boundaries typically involve powers of $C^{4}$, in which case (48) can be conveniently written in terms of the $O(\lambda)$ angle $\theta$ defined in (47):

$$
C^{4 l}=\cos (4 l \theta)\left(\begin{array}{ll}
1 & 0 \\
0 & 1
\end{array}\right)-\frac{\sin (4 l \theta)}{\sqrt{4-\lambda^{2}}}\left(\begin{array}{cc}
\lambda & -2 \\
2 & -\lambda
\end{array}\right) .
$$

Once the segments $\xi_{m}$ have been determined, the indicated intersections are calculated by elementary algebra (a tedious exercise, best done with computer assistance [12]). With $M$ as given in (29), we have

$$
\begin{aligned}
P_{0}(m)= & \left(\frac{1}{2}\left(1+2 \lambda+\frac{\tan \theta}{\tan (2 m+1) \theta}\right), 1\right), \quad 1 \leq m \leq M, \\
P_{1}(m)= & \left(\frac{1}{2}\left(1+2 \lambda-\lambda^{2}+\lambda^{4}+\left(1-3 \lambda^{2}+\lambda^{4}\right) \frac{\tan \theta}{\tan (2 m-1) \theta}\right),\right. \\
& \left.1+\frac{\lambda^{3}}{2}+\frac{\lambda}{2}\left(\lambda^{2}-2\right) \frac{\tan \theta}{\tan (2 m-1) \theta}\right), \quad 2 \leq m \leq M .
\end{aligned}
$$


We recall that for $m=1,2$, the symmetry axis of $\Lambda_{m}^{\text {out }}$ intersects $\mathbf{0}$ instead of $\mathbf{5}^{1}$, and $P_{1}(m)$ was defined to be the right-hand endpoint of the symmetry axis (see remarks preceding equation (39), and theorem (11). The atom $\Lambda_{1}^{\text {out }}$ is a quadrilateral (see equation 37), with vertices

$$
\Lambda_{1}^{\text {out }}=\left[P_{0}(1),(1,1), P_{1}(0), P^{\prime}\right]
$$

where

$P_{1}(0)=\left(1, \frac{2-\lambda-\lambda^{2}}{2-4 \lambda^{2}+\lambda^{4}}\right), \quad P^{\prime}=\left(1-2 \lambda^{2}+6 \lambda^{3}+\lambda^{4}-5 \lambda^{5}+\lambda^{7}, 1-\lambda+3 \lambda^{2}+\lambda^{3}-4 \lambda^{4}+\lambda^{6}\right)$.

The atom $\Lambda_{2}^{\text {out }}$ is the hexagon

$$
\Lambda_{2}^{\text {out }}=\left[P_{0}(2), P_{0}(1), P^{\prime \prime}, P_{1}(1), P^{\prime \prime \prime}, P_{1}(2)\right]
$$

where

$$
\begin{aligned}
P_{1}(1)= & \left(1, \frac{2-2 \lambda-4 \lambda^{2}+\lambda^{3}+\lambda^{4}}{2-9 \lambda^{2}+6 \lambda^{4}-\lambda^{6}}\right), \quad P^{\prime \prime}=\left(1, \frac{1-\lambda-3 \lambda^{2}+\lambda^{3}+\lambda^{4}}{1-6 \lambda^{2}+5 \lambda^{4}-\lambda^{6}}\right), \\
P^{\prime \prime \prime}= & \left(\frac{1-6 \lambda^{2}-3 \lambda^{3}+11 \lambda^{4}+4 \lambda^{5}-12 \lambda^{6}-\lambda^{7}+6 \lambda^{8}-\lambda^{10}}{1-6 \lambda^{2}+5 \lambda^{4}-\lambda^{6}},\right. \\
& \left.\frac{1-\lambda-4 \lambda^{2}+3 \lambda^{3}+4 \lambda^{4}-7 \lambda^{5}-\lambda^{6}+5 \lambda^{7}-\lambda^{9}}{1-6 \lambda^{2}+5 \lambda^{4}-\lambda^{6}}\right) .
\end{aligned}
$$

To calculate the vertices $Q_{0}(n)$ and $Q_{1}(n)$ of the regular atoms of $L^{\text {in }}$, we apply to the line $\gamma_{0}=\mathbf{1}$ a $4 n$-fold $C$-rotation about the centre of $\mathcal{E}$, followed by a $G$-reflection and, finally, intersection with the lines $\gamma_{0}$ and $\gamma_{1}=\mathbf{5}^{1}$, respectively.

The results are [12]

$$
\begin{aligned}
Q_{0}(n) & =\left(\frac{2-\lambda-\lambda^{2}-s_{n}(\lambda)+(2+\lambda) c_{n}(\lambda)}{-\lambda s_{n}(\lambda)+\left(4-\lambda^{2}\right) c_{n}(\lambda)}, 1\right) \\
Q_{1}(n) & =\left(\frac{a(\lambda)+b(\lambda) s_{n}(\lambda)+c(\lambda) c_{n}(\lambda)}{d(\lambda) s_{n}(\lambda)+e(\lambda) c_{n}(\lambda)}, \frac{f(\lambda)+g(\lambda) s_{n}(\lambda)+h(\lambda) c_{n}(\lambda)}{d(\lambda) s_{n}(\lambda)+e(\lambda) c_{n}(\lambda)}\right)
\end{aligned}
$$

with

$$
\begin{array}{ll}
a(\lambda)=2-\lambda-7 \lambda^{2}+3 \lambda^{3}+5 \lambda^{4}-\lambda^{5}-\lambda^{6} & b(\lambda)=-1-2 \lambda-\lambda^{2}+2 \lambda^{3}+\lambda^{4} \\
c(\lambda)=2+\lambda-6 \lambda^{2}-3 \lambda^{3}+2 \lambda^{4}+\lambda^{5} & d(\lambda)=-\lambda\left(5-5 \lambda^{2}+\lambda^{4}\right) \\
e(\lambda)=\left(4-\lambda^{2}\right)\left(1-3 \lambda^{2}+\lambda^{4}\right) & f(\lambda)=-\lambda\left(4-2 \lambda-4 \lambda^{2}+\lambda^{3}+\lambda^{4}\right) \\
g(\lambda)=\lambda\left(-3-\lambda+2 \lambda^{2}+\lambda^{3}\right) & h(\lambda)=4-7 \lambda^{2}-3 \lambda^{3}+2 \lambda^{4}+\lambda^{5} .
\end{array}
$$

and

$$
\begin{aligned}
& c_{k}(\lambda)=\cos (4 k \theta)=T_{4 k}\left(\frac{\lambda}{2}\right) \\
& s_{k}(\lambda)=\sqrt{4-\lambda^{2}} \sin (4 k \theta)=\left(\frac{\lambda^{2}-4}{2}\right) U_{4 k-1}\left(\frac{\lambda}{2}\right) .
\end{aligned}
$$


Here $T_{k}$ and $U_{k}$ are the Chebyshev polynomials of the first and second kinds, respectively $[19, \mathrm{p}$ 1032].

The use of the rotation angle $4 n \theta$ in (49), as well as in the formulae for $Q_{0}(n)$ and $Q_{1}(n)$, allows for a natural interpolation of these quantities in the range $0 \leq n \leq N-1$. For continuously varying $n$, the matrix $C^{4 n}$ defined by (49) (not (48)) represents a family of $C$-rotations whose angle increases monotonically from 0 to $4 N \theta=\pi / 2-O(\lambda)$. Moreover, as $n$ increases from 0 to $N-1, Q_{0}(n)$ and $Q_{1}(n)$ smoothly trace out, in order-preserving fashion, the upper and lower boundaries of the regular part of $\Lambda$.

Note that formulae (54) and (55) explicitly specify the invertible transformations between parameter $n$ and points $X=(x, y)$ on the lines $\gamma_{0}$ and $\gamma_{1}$, respectively. This gives precise meaning to the functional notation $n(X), X \in \Sigma$, which we will employ below. In section 5 we will exploit this parametrization to study the relative positions on the two boundary lines of the points $P_{0}(m)$ and $Q_{0}(n)$ (resp. $P_{0}(m)$ and $Q_{0}(n)$ ).

\subsection{A preliminary lemma}

The following lemma plays an important role in the theorem which follows.

Lemma 12 The map $F^{4}$ is an isometry on the triangle $\left[(0,1),(1,1),\left(\lambda^{2}, 1-\lambda\right)\right]$, with itinerary

$$
\begin{aligned}
& {\left[(0,1),(1,1),\left(\lambda^{2}, 1-\lambda\right)\right] \stackrel{F_{7}}{\rightarrow}\left[(0,0),(0,1),\left(\lambda, \lambda^{2}\right)\right] \stackrel{F_{1}}{\rightarrow}[(0,0),(1, \lambda),(1,0)]} \\
& \quad \stackrel{F_{0}}{\rightarrow}[(0,0),(0,1),(\lambda, 1)] \stackrel{F_{7}}{\rightarrow}\left[(0,0),\left(\lambda^{2}, \lambda\right),(1,0)\right] .
\end{aligned}
$$

The triangles $F^{k}\left(\left[(0,1),(1,1),\left(\lambda^{2}, 1-\lambda\right)\right]\right), k=1, \ldots, 4$, do not intersect the sector $\Sigma$.

Proof. The proof is by direct mapping of the vertices, accompanied by straightforward verification that the interior each triangle is contained in a single atom of $F$, the latter being correctly identified in the statement of the lemma. The empty intersection of the four image triangles with $\Sigma$ is trivially verified.

\subsection{Algebraic alternative to theorem 11}

We now state an alternative to theorem 11 in which the polygons $\Lambda_{m}^{\text {out }}$ are defined explicitly in terms of their respective vertices, as in section 4.1. That these are symmetric atoms of $L^{\text {out }}$ is proved by an inductive calculation of their return paths.

Theorem 13 For $1 \leq m \leq M$, we have

$$
L^{\text {out }}\left(\Lambda_{m}^{\text {out }}\right)=F^{4 m+3} \circ G\left(\Lambda_{m}^{\text {out }}\right)=F_{\left(1^{3}(0,1)^{2 m-1} 1^{2}\right)} \circ G\left(\Lambda_{m}^{\text {out }}\right)=\Lambda_{m}^{\text {out }} .
$$


Moreover,

$$
\left[P_{0}(m), P_{1}(m-1)\right]=\text { Fix } L_{m}^{\text {out }}
$$

Proof. We begin by defining some auxiliary polygons related to $\Lambda_{m}^{\text {out }}$ :

$$
\begin{aligned}
& \hat{\Lambda}_{m}^{\text {out }} \stackrel{\text { def }}{=} \bigcup_{k \geq m}^{M} \Lambda_{m}^{\text {out }} \cup\left[P_{0}(M), P_{1}(M),(g, 1)\right], \\
& \check{\Lambda}_{m}^{\text {out }} \stackrel{\text { def }}{=} F_{\left(1^{3}(0,1)^{2 m-1}\right)} \circ G\left(\hat{\Lambda}_{m}^{\text {out }}\right) .
\end{aligned}
$$

We want to prove that $G\left(\hat{\Lambda}_{m}\right)$ is mapped isometrically onto $\check{\Lambda}_{m}$ by $F^{4 m+1}$.

The proof is by induction on $m$. For $m=1$, the isometric property follows from lemma 12 by showing that $F\left(G \hat{\Lambda}_{1}^{\text {out }}\right)$ is contained in $\left[(0,1),(1,1),\left(\lambda^{2}, 1-\lambda\right)\right]$. To verify this, we note that

$$
F\left(G \hat{\Lambda}_{1}^{\text {out }}\right) \subset F\left(\left[\left(g^{\prime \prime}, 1\right),(1,1),(1, g)\right]\right)=\left[\left(\lambda g^{\prime \prime}, g^{\prime \prime}\right),(\lambda, 1),(1+\lambda-g, 1)\right] .
$$

It is a simple exercise [12] (sec. E.5.1)to show that for $0<\lambda<1$, all three vertices of the image triangle are within or on the boundary of $\left[(0,1),(1,1),\left(\lambda^{2}, 1-\lambda\right)\right]$.

We now assume that the induction hypothesis $F^{4 m+1} \circ G\left(\hat{\Lambda}_{m}\right)=\check{\Lambda}_{m}$ holds for a given $m<M$. Note that $G\left(\hat{\Lambda}_{m}\right)$ is partitioned by the line segment $G\left(\left[P_{0}(m), P_{1}(m)\right]\right)(m>1)$ or $G\left(\left[P_{0}(1), P^{\prime}\right]\right)(m=1)$ into adjacent polygons $G\left(\Lambda_{m}^{\text {out }}\right)$ and $G\left(\hat{\Lambda}_{m+1}\right)$. We denote the images of these polygons $\check{\Lambda}_{m}^{+}$and $\check{\Lambda}_{m}^{-}$,respectively. One checks [12] (secs. E.5.2, E.5.3) that the boundary separating the two is a subset of $[(0,0),(1, \lambda)]$, so that

$$
\check{\Lambda}_{m}^{-} \subset \Omega_{0}, \quad \check{\Lambda}_{m}^{+} \subset\left(\left[(0,0),\left(\lambda^{2}, \lambda\right),(1,0)\right] \backslash \Omega_{0}\right) \subset\left(\Omega_{1} \backslash \Sigma\right) .
$$

To complete the proof of

$$
F^{4 m+1} \circ G\left(\hat{\Lambda}_{m+1}\right)=\check{\Lambda}_{m+1}
$$

we need to show that $F^{4}$ maps $\check{\Lambda}_{m}^{-}$isometrically onto $\check{\Lambda}_{m+1}$, with itinerary $(0,1,0,1)$. Using lemma 12 and $\check{\Lambda}_{m}^{-} \subset \Omega_{0}$, we have

$$
F^{2}\left(\check{\Lambda}_{m}^{-}\right)=F_{(01)}\left(\check{\Lambda}_{m}^{-}\right) \subset \Omega_{0},
$$

where the inclusion is verified explicitly [12] (sec. E.5.4). Again using lemma 12, we have

$$
F^{4}\left(\check{\Lambda}_{m}^{-}\right)=F_{(0101)}\left(\check{\Lambda}_{m}^{-}\right)
$$

The right-hand side is identical to $\check{\Lambda}_{m+1}$ by construction.

Having established (56), we need only show that $F^{2}$ maps $\check{\Lambda}_{m}^{+}$isometrically onto $\Lambda_{m}^{\text {out }}$ with itinerary $(1,1)$. For the isometry, we verify the inclusion [12] (sec. E.5.5)

$$
F_{1}\left(\check{\Lambda}_{m}^{+}\right) \subset\left(\Omega_{1} \backslash \Sigma\right)
$$


The rest is an explicit calculation [12] (sec. E.6) of the image points of the vertices, using elementary algebra, In particular, we find that the map $L_{m}^{\text {out }}=F^{4 m+3} \circ G$ acts on the vertices of the polygons $\Lambda_{m}^{\text {out }}$ as follows (notation of section 4.1):

$$
\begin{aligned}
& P_{0}(m) \mapsto P_{0}(m), \quad P_{1}(m-1) \mapsto P_{1}(m-1), \quad 1 \leq m \leq M, \\
& P_{0}(m-1) \leftrightarrow P_{1}(m), \quad 2 \leq m \leq M, \\
& (1,1) \leftrightarrow P^{\prime}, \quad m=1, \quad P^{\prime \prime} \leftrightarrow P^{\prime \prime \prime}, \quad m=2 .
\end{aligned}
$$

The final identification of $F^{4 m+3} \circ G$ as the first-return map $L^{\text {out }}$, with regular atoms $\Lambda_{m}^{\text {out }}$ requires verification that all polygons $F^{k} \circ G\left(\Lambda_{m}^{\text {out }}\right), 1 \leq k<4 m+3$, are disjoint from $\Sigma$. We have taken care to establish this property at each stage of the proof (specifically, in lemma 12 and equations (57) and (58) ).

Recalling that the map $L^{\text {out }}$ acts linearly on $\Lambda_{m}^{\text {out }}$, the vertex mappings are sufficient to establish that the atom is $L^{\text {out }}$-symmetric with fixed segment $\left[P_{0}(m), P_{1}(m-1)\right]$.

\section{$5 \quad$ Regular atoms and fixed points of $L$}

\subsection{Overview}

In section 3 we decomposed the return map $L$ into a product of involutions $L^{\text {in }}$ and $L^{\text {out }}$. According to theorem $6, L^{\text {in }}$ is endowed with a sequence of regular atoms $\Lambda_{n}^{\text {in }}, n=$ $1, \ldots, N-1$, each of which is an $L^{\text {in }}$-symmetric polygon. Theorem 11 establishes an analogous sequence of regular atoms, $\Lambda_{m}^{\text {out }}, m=1, \ldots, M$ for $L^{\text {out }}$. We now define regular atoms of $L$ to be the nontrivial intersections of the regular atoms of the two involutions:

$$
\Lambda_{m, n}=\Lambda_{m}^{\text {out }} \cap \Lambda_{n}^{\text {in }}
$$

For some, but not necessarily all, of these atoms, the symmetry lines of $L^{\text {in }}$ and $L^{\text {out }}$ in $\Lambda_{n}^{\text {in }}$ and $\Lambda_{m}^{\text {out }}$, respectively, intersect within $\Lambda_{m, n}$, at a symmetric return-map fixed point $Z(m, n)$. Such fixed points will be called regular, and will be the main focus of this and succeeding sections. They correspond to a 2-parameter family of periodic orbits of $F$, with periods

$$
t(m, n)=4(m+n)-1
$$

and symbolic codes

$$
\iota^{(n, m)}=\left(\overline{1^{4 n-1},(0,1)^{2 m-1}, 1,1}\right),
$$

the latter obtained by concatenating the codes of the involutions $L^{\text {in,out }}$ given in theorems 6. and 11 .

Before examining in detail the geometric properties of the regular atoms of $L$, it is useful to introduce a broad classification according to the size of the index $m$ relative to 

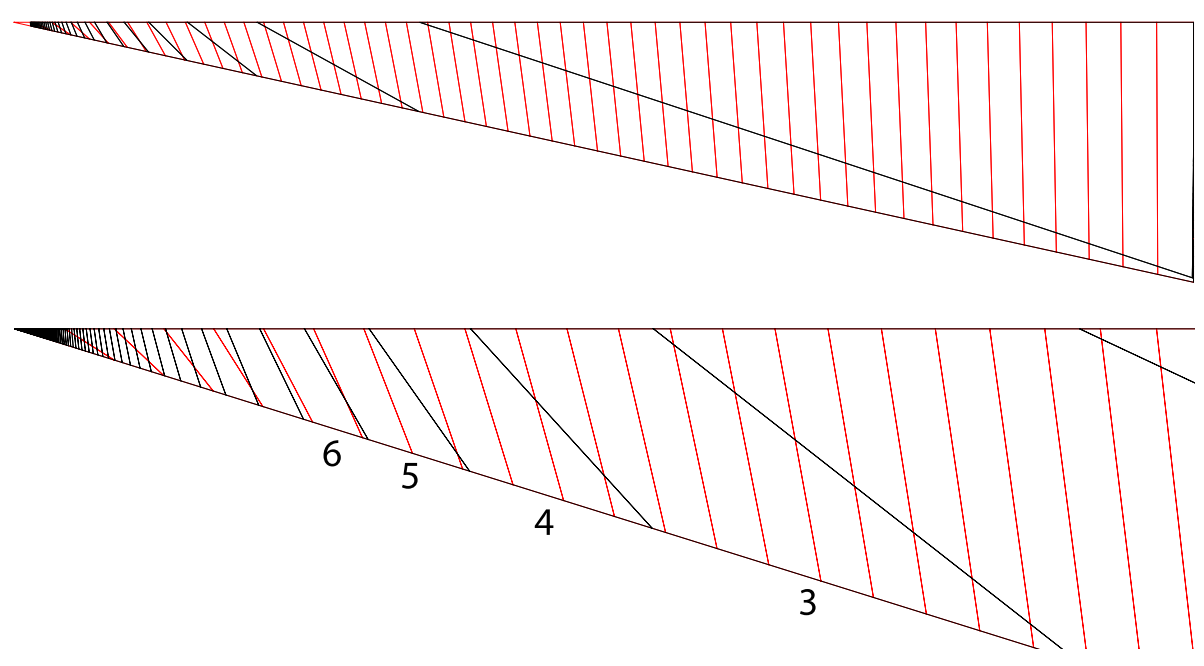

Figure 7: Top: Superposition of the atoms of the domain $\Lambda$ into atoms. The atoms of $L^{\text {in }}$ are in red; those of $L^{\text {out }}$ in black. Bottom: Magnification of the region near the tip of $\Lambda$, showing the crossover phenomenon. The integers denote values of $m$, and crossover occurs for $m=6$.

its maximum value $M=M(\lambda)$, given by equation (29). A typical example is displayed in figures 7 and 8 , where $\lambda$ has been assigned the value $\frac{1}{64}$. Figure 7 shows the regular atoms of $L^{\text {in }}$ (lighter boundaries) and $L^{\text {out }}$ (darker boundaries) in all of $\Lambda$ (note that the aspect ratio has purposely been distorted to make the structural relationships visible). The bottom figure 17 zooms in on the left-hand end of $\Lambda$, while figure 8 zooms in on the atoms with $m=3,30 \leq n \leq 40$, showing explicitly Fix $L^{\text {in }}$ and Fix $L^{\text {out }}$ and the regular fixed points of the return-map where these symmetry lines intersect.

We observe some interesting features of the regular atoms and their symmetry lines. The orientations of the 'vertical' boundary lines change substantially as one traverses $\Lambda$. The $\Lambda_{n}^{\text {in }}$ boundaries are vertical at the right-hand edge but suffer significant counterclockwise rotations as one moves toward the left-hand vertex. The $\Lambda_{n}^{\text {out }}$ boundaries, on the other hand, rotate in the opposite sense. Somewhere in the middle, there is a crossover region where the boundaries of the two sets of atoms are approximately aligned (this corresponds to $m=6$ in figure 7). To the right of the crossover region, each $\Lambda_{m}^{\text {out intersects }}$ a number of successive $\Lambda_{n}^{\text {in }}$ atoms, and each of the intersections $\Lambda_{m, n}$ (the regular atoms of $L$ ) contains a unique regular fixed point $Z(m, n)$. To the left of the crossover region, on the other hand, each $\Lambda_{m}^{\text {in }}$ intersects a number of successive $\Lambda_{n}^{\text {out }}$ atoms, and now there are regular atoms $\Lambda_{m, n}$ which do not contain a regular fixed point. This can be seen quite clearly in the case $m=8$, which has a triangular intersection region in its northeast corner which is not intersected by the diagonal Fix $L_{8}^{\text {out }}$.

In appendix A.7 we give a precise definition of the crossover point $m=m^{*}, n=n^{*}$ and obtain a perturbative formula for $m^{*}$ and $n^{*}$ as functions of $\lambda$. In particular, we will find

$$
m^{*}=\frac{1}{\sqrt{2 \lambda}}\left(1+\frac{\lambda}{3}+\cdots\right), \quad n^{*}=\frac{\pi}{4 \lambda}-\frac{1}{\sqrt{2 \lambda}}-\frac{3}{4}+\cdots .
$$

We note that in the limit $\lambda \rightarrow 0^{+}$, the crossover value of $m$ tends to infinity. Thus, if 


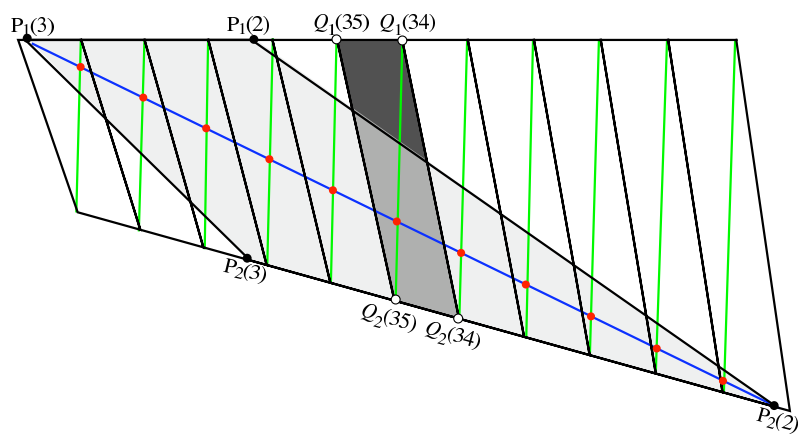

Figure 8: The fixed points of the return map $L$ are the intersection of the fixed lines of the involutions $L^{\text {in,out}}$, which are segments connecting opposite vertices of the atoms $\Lambda_{n}^{\text {in }}$ (vertices $\left.Q_{0}(n), Q_{0}(n-1), Q_{1}(n-1), Q_{1}(n)\right)$, and $\Lambda_{m}^{\text {out }}$ (vertices $\left.P_{0}(m), P_{0}(m-1), P_{1}(m-1), P_{1}(m)\right)$. The example focuses on $m=3, n=34$ for $\lambda=\frac{1}{64}$.

we are interested in the asymptotic behaviour of regular fixed points and their stability ellipses for fixed positive integer $m$, we can limit our attention to those regular atoms which are well to the right of the crossover, and avoid the complexities of the crossover phenomenon. This is achieved by introducing a cut-off condition

$$
m \leq \lambda^{-\nu_{0}}, \quad \text { where } \quad 0<\nu_{0}<\frac{1}{2} .
$$

We will prove that the regular atoms satisfying this condition have a number of nice properties, which allow us to obtain explicit formulae for their total area, whereas those with $m$ exceeding the cut-off have a total area tending to zero as $\lambda^{\frac{1}{2}-\nu_{0}}$, and can therefore be neglected.

In the remainder of this section, we study in detail the properties of the regular atoms of $L$. Our starting point is the list of explicit formulae for the atomic vertices given in section 4.1. The vertex coordinates are rational functions of $\lambda$; as $\lambda \rightarrow 0$, we will consider Taylor expansions in $\lambda$, with suitable remainder. Our most complete description of the atoms $\Lambda_{m, n}$ will be restricted to those satisfying cut-off conditions $\lambda \leq \lambda_{0}, m \leq \lambda^{-\nu_{0}}$. Our choice of cut-off parameters is (cf. (62))

$$
\lambda_{0}=10^{-4} \quad \text { and } \quad \nu_{0}=\frac{1}{4}
$$

Even though a specific choice is required to obtain explicit estimates, our qualitative results are insensitive to these special values, and the detailed calculations included in the Electronic Supplement [12] can readily be adapted to other cut-off values. For the rest of this section, we shall assume that $\lambda_{0}$ and $\nu_{0}$ are given by (63).

The organization of the regular atoms of $L^{\text {in,out }}$ and their intersections $\Lambda_{m, n}$ is determined by the order and spacing of the vertices along the lines $\mathbf{1}$ and $\mathbf{5}^{1}$. Often it will be convenient to regard $n$ as a continuous variable on the interval $[0, N]$, as discussed in section 4.1, and to express our results in terms of auxiliary variables

$$
\eta=\frac{\pi}{2}-2 n \theta, \quad \tau=\tan (\eta)
$$


Note that as $n$ increases from 0 to $N$, the quantity $\tau$ decreases monotonically from 1 to $\tan (\pi / 4-2 N \theta)=O(\lambda)$.

In section 4.1, we introduced the notation $n(X)$ to designate the real parameter $n$ assigned to a point $X$ on one of the lines $\mathbf{1}$ or $\mathbf{5}^{1}$. In the same spirit, we will employ $\tau(X)$ to denote the corresponding $\tau$-variable, namely $\tan (\pi / 4-2 n(X) \theta)$.

\subsection{Expansions for the atom vertices}

We begin our structural analysis by determining the positions of the vertices $P_{0,1}(m), Q_{0,1}(n)$, correct to next-to-leading order in powers of $\lambda$. The following lemma establishes, among other things, that the horizontal positions and widths of the atoms $\Lambda_{m}^{\text {out }}$ become fixed as $\lambda$ tends to zero, while the vertical dimension shrinks proportional to $\lambda$. For the atoms $\Lambda_{n}^{\text {in }}$, on the other hand, both dimensions shrink proportional to $\lambda$. In addition, we see that the $x$-coordinates of $P_{0}(m-1)$ and $P_{1}(m)$ coincide up to first order in $\lambda$. These properties are apparent in the figures. The proof of the lemma is given in appendices A.2 and A.3.

Lemma 14 For $\lambda \leq \lambda_{0}$, and $2 \leq m \leq \lambda^{-\nu_{0}}$, with $\lambda_{0}, \nu_{0}$ given in (63), the cartesian coordinates of the vertices of the regular atoms $\Lambda_{m}^{\text {out }}$ and $\Lambda_{n}^{\text {in }}$ are given by

$$
\begin{aligned}
P_{0}(m)= & \left(\frac{m+1}{2 m+1}+\lambda-\frac{1}{2 m+1}\left(\frac{m(m+1)}{6} \lambda^{2}+r_{0 x} \lambda^{3}\right), 1\right) \\
P_{1}(m)= & \left(\frac{m}{2 m-1}+\lambda-\frac{1}{2 m-1}\left(\frac{(m+2)(m+3))}{6} \lambda^{2}+r_{1 x} \lambda^{3}\right),\right. \\
& \left.1+\frac{1}{2 m-1}\left(-\lambda+\frac{m(m+2)}{3} \lambda^{3}+r_{1 y} \lambda^{4}\right)\right) \\
Q_{0}(n)= & \left(\frac{1}{2}(1+\tau)+\frac{\lambda}{8}\left(3-2 \tau-\tau^{2}\right)+\frac{\lambda^{2}}{32}\left(2-5 \tau+2 \tau^{2}+\tau^{3}\right)+r_{2 x} \lambda^{3}, 1\right) \\
Q_{1}(n)= & \left(\frac{1}{2}(1+\tau)+\frac{\lambda}{8}\left(7-2 \tau-5 \tau^{2}\right)+\frac{\lambda^{2}}{32}\left(-6-29 \tau+10 \tau^{2}+25 \tau^{3}\right)+r_{3 x} \lambda^{3},\right. \\
& \left.1-\tau \lambda+\frac{\lambda^{2}}{4}\left(1+2 \tau+5 \tau^{2}\right)-\frac{\lambda^{3}}{16}\left(2+11 \tau+10 \tau^{2}+25 \tau^{3}\right)+r_{3 y} \lambda^{4}\right),
\end{aligned}
$$

where $\tau$ is defined in (64), and the remainders $r$ are rational functions of $\lambda^{1 / 2}$ that admit the following bounds

$$
\begin{aligned}
& r_{0 x} \in\left[-\frac{1697}{10^{8}}, \frac{1507}{10^{5}}\right], \quad r_{1 x} \in\left[-\frac{8523}{10^{6}}, \frac{1243}{10^{5}}\right], \quad r_{1 y} \in\left[-\frac{5449}{10^{6}}, \frac{1203}{50000}\right], \\
& r_{2 x} \in\left[-\frac{2051}{5000}, \frac{4499}{10000}\right], \quad r_{3 x} \in\left[-\frac{23}{20}, \frac{389}{250}\right], \quad r_{3 y} \in\left[\frac{519}{5000}, \frac{539}{100}\right] .
\end{aligned}
$$

In addition, we have

$$
\begin{array}{ll}
P_{0}(1)=\left(\frac{2}{3}+\lambda-\frac{1}{9} \lambda^{2}+r_{01 x} \lambda^{4}, 1\right), & r_{01 x} \in\left[-\frac{463}{12500},-\frac{3703}{100000}\right] \\
P_{1}(0)=\left(1,1-\frac{1}{2} \lambda+\frac{3}{2} \lambda^{2}-\lambda^{3}+r_{10 y} \lambda^{4}\right), & r_{10 y} \in\left[\frac{1249}{500}, \frac{313}{125}\right] \\
P_{1}(1)=\left(1,1-\lambda+\frac{5}{2} \lambda^{2}-4 \lambda^{3}+r_{11 y} \lambda^{4}\right), & r_{11 y} \in\left[\frac{8739}{1000}, \frac{8763}{1000}\right] .
\end{array}
$$


Corollary 15 For $\lambda \leq \lambda_{0}$ and $2 \leq m \leq \lambda^{-\nu_{0}}$, we have

$$
P_{0}(m)_{x}<P_{1}(m)_{x}<P_{0}(m-1)_{x}<P_{1}(m-1)_{x}
$$

with

$$
P_{1}(m)_{x}-P_{0}(m)_{x}=O(1), \quad P_{1}(m)_{x}-P_{0}(m-1)_{x}=O\left(\lambda^{2}\right) .
$$

For $m=1$,

$$
P_{0}(1)<P_{1}(0), \quad P_{1}(0)-P_{0}(1)=O(1)
$$

In addition to the information about the horizontal ordering of cartesian coordinates, we will need analogous results with respect to $n$, considered as a continuous variable. The proof is found in appendix A.5.

Lemma 16 For $\lambda \leq \lambda_{0}$ and $2 \leq m \leq \lambda^{-\nu_{0}}$, we have

$$
n\left(P_{0}(m)\right)>n\left(P_{1}(m)\right)>n\left(P_{0}(m-1)\right)>n\left(P_{1}(m-1)\right),
$$

and the quantities $\lambda\left(n\left(P_{0}(m)\right)-n\left(P_{1}(m)\right)\right)$ and $\lambda^{-1}\left(n\left(P_{1}(m)\right)-n\left(P_{0}(m-1)\right)\right)$ have positive lower and upper bounds which are independent of $\lambda$. In the case $m=1$, we have

$$
n\left(P_{0}(1)\right)>n\left(P_{1}(0)\right)=0,
$$

and the quantity $\lambda\left(n\left(P_{0}(1)\right)-n\left(P_{1}(0)\right)\right)$ has positive lower and upper bounds which are independent of $\lambda$.

\subsection{Structural theorem}

We are now in a position to establish the most important structural properties of the regular atoms of the return-map $L$.

Theorem 17 If the inequalities

$$
P_{0}(m)_{x}<Q_{0}(n-1)_{x} \quad \text { and } \quad P_{1}(m-1)_{x}>Q_{1}(n)_{x}
$$

are satisfied for some integers $m, n$ in the range $1 \leq m \leq M, 1 \leq n<N$, then the regular atom $\Lambda_{m, n}=\Lambda_{n}^{\text {in }} \cap \Lambda_{m}^{\text {out }}$ has positive area, and contains a regular fixed point $Z(m, n)$. The stability ellipse $\mathcal{E}_{m, n}$ surrounding $Z(m, n)$ is tangent to (at least) three sides of $\Lambda_{m, n}$, one of which is a subset of either $\mathbf{1}$ or $\mathbf{5}^{\mathbf{1}}$.

Given $0<\lambda \leq \lambda_{0}$ and $1 \leq m \leq \lambda^{-\nu_{0}}$, then there is a non-empty set of values of $n$ for which the inequalities (68) hold, given by

$$
n_{-}(m) \leq n \leq n_{+}(m), \quad n_{-}(m)=\left\lceil n\left(P_{1}(m-1)\right)\right\rceil, \quad n_{+}(m)=\left\lceil n\left(P_{0}(m)\right)\right\rceil .
$$

As $\lambda \rightarrow 0$, such a set becomes infinite. 
Proof. From equations (24) and (39) we see that the points $P_{0}(m)$ and $Q_{0}(n-1)$ belong to the segment $\mathbf{1}$, which is parallel to the $x$-axis. Suppose that $m>2$. Then the points $P_{1}(m-1)$ and $Q_{1}(n)$ belong to $\mathbf{5}^{1}$, which is quasi-parallel to the $x$-axis. Then the inequalities (68) imply a corresponding ordering of the $P_{\mathrm{s}}$ and $Q_{\mathrm{s}}$ along the respective segments. It follows that the segments $\left[P_{0}(m), P_{1}(m-1)\right]$ and $\left[Q_{0}(n-1), Q_{1}(n)\right]$ intersect transversally at a point $Z(m, n)$, which lies in the interior of both atoms, hence in the interior of their intersection. This establishes the existence of a regular atom $\Lambda_{m, n}$ of positive measure. From theorems 6 and 11 we recognize $\left[P_{0}(m), P_{1}(m-1)\right]$ as the symmetry axis for $L_{m}^{\text {out }}$, and $\left[Q_{0}(n-1), Q_{1}(n)\right]$ as the symmetry axis for $L_{n}^{\text {in }}$, so that the intersection point $Z(m, n)$ is indeed a regular fixed point of $L$. If $m=1,2$, the symmetry axis of $L_{1,2}^{\text {out }}$ connects $P_{0}(m)$ to $\mathbf{0}$; the second inequality in (68) is automatically satisfied, and the first inequality in (68) suffices to establish transversal intersection of symmetry lines.

Because the atoms $\Lambda_{m}^{\text {out }}$ and $\Lambda_{n}^{\text {in }}$ are maximal, so is $\Lambda_{m, n}$. Since the fixed point $Z(m, n)$ lies in the interior of $\Lambda_{m, n}$, the associated ellipse $\mathcal{E}_{m, n}$ is necessarily tangent to the boundary of $\Lambda_{m, n}$. In particular, $Z(m, n)$ is stable.

We must now establish the number of points of tangency. Let $T$ be a point at which $\Lambda_{m, n}$ is tangent to $\mathcal{E}_{m, n}$. To make our argument independent from the details of the boundary, we use the expression $L_{m, n}^{\text {in,out }}(T)$ to denote the limit of $L_{m, n}^{\text {in,out }}(z)$ as $z$ approaches $T$ from the interior of the atom. Now, any $L$-invariant ellipse in the interior of $\mathcal{E}_{m, n}$ is also invariant under the individual involution $L_{n}^{\text {in }}$ and $L_{m}^{\text {out }}$. To see this, note that, generically (irrational rotation number), such an ellipse contains a symmetric dense orbit, which is mapped into itself by one involution, hence by the other. By continuity, this result then extends to the non-generic case (rational rotations). Because of this property, if $T$ is tangent to $\mathcal{E}$, so are $L_{n}^{\text {in }}(T)$ and $L_{m}^{\text {out }}(T)$.

Let us assume that $(m, n) \neq(1,1)$ or $(2,1)$, which are special cases to be dealt with separately. We distinguish three possibilities, depending on whether $\Lambda_{m, n}$ is tangent to $\mathbf{5}$, to $\mathbf{1}$, or to both2 (see figure 8). If $\Lambda_{m, n}$ is tangent only to $\mathbf{5}$, then $T \notin-\mathbf{4 n}+\mathbf{4}$, because $L_{n}^{\text {in }}(-\mathbf{4 n}+\mathbf{4})=\mathbf{1}$, which lies outside the closure of the atom. The two involutions exchange the remaining three boundaries as follows

$$
-4 \mathbf{n} \stackrel{L_{n}^{\text {in }}}{\longleftrightarrow} \mathbf{5} \stackrel{L_{m}^{\text {out }}}{\longleftrightarrow} 4 \mathbf{m}-\mathbf{1}
$$

and since the images of $T$ lie on the boundary of $\mathcal{E}$, we get one tangency point on each of the above three sides. In particular, if $\Lambda_{m, n}$ is a triangle, then $\mathcal{E}_{m, n}$ is tangent to all its sides. In the case $(m, n)=(2,1)$, the atom $\Lambda_{2,1}$ has the additional involutory relation $\mathbf{1 2}=L_{1}^{\text {out }}(\mathbf{0})$. Since $T$ cannot be tangent to $\mathbf{0}$, then it cannot be tangent to $\mathbf{1 2}$. But then $\Lambda_{2,1}$ is necessarily a pentagon (i.e., $\mathbf{5}$ is a side of the atom), for otherwise the remaining two sides would map outside the atom, and we would have no tangency, contradicting maximality. So we still get three points on $\mathbf{5 ,} \mathbf{7}$ and $\mathbf{1 2}$.

An analogous argument applies to the case in which $\Lambda_{m, n}$ is tangent to 1 but not to 5. Now the ellipse $\mathcal{E}$ cannot be tangent to $\mathbf{- 4 n}$, and the involutions act on the remaining

\footnotetext{
${ }^{2}$ To lighten up the notation, we'll omit all superscripts identifying regular components.
} 
sides as follows

$$
-4 \mathbf{n}+4 \stackrel{L_{n}^{\text {in }}}{\longleftrightarrow} \quad \mathbf{1} \stackrel{L_{m}^{\text {out }}}{\longleftrightarrow} 4 \mathbf{m}+\mathbf{3}
$$

giving again one tangency point on each of the above three sides. In the special cases $(m, n)=(1,1)$, we have the additional involutory relation $\mathbf{8}=L_{1}^{\text {out }}(\mathbf{0})$. Again, $\Lambda_{1,1}$ is necessarily a pentagon (lest we would have no tangency). This time the ellipse $\mathcal{E}_{1,1}$ is tangent to $\mathbf{0}$, and so we obtain a fourth point of tangency to $\mathbf{8}$.

It remains to consider the case in which $\Lambda_{m, n}$ is tangent to both $\mathbf{1}$ and $\mathbf{5}$. We cannot have the quadrilateral atom $\Lambda_{m, n} \neq \Lambda_{n}^{\text {in }}$, because its boundaries $\mathbf{1}$ and $\mathbf{5}$ are mapped outside the closure of the atom by $L_{m}^{\text {out }}$, and mapped to the other two boundaries by $L_{n}^{\text {in }}$. But this would imply the absence of tangency points, contrary to the maximality of the atom. For a similar reason $\Lambda_{m, n} \neq \Lambda_{m}^{\text {out }}$. Thus $\Lambda_{m, n}$ is either a pentagon or a hexagon. In the former case, symmetry still forces the same three tangency points as above, one of which is either $\mathbf{1}$ or $\mathbf{5}$. However, $\mathcal{E}$ cannot be tangent to both, since one of these sides is mapped outside the atom by $L_{m}^{\text {out }}$. In the hexagonal case there are two possible configurations with three tangency points, of which at least one must be realized. Each configuration must be realized in some parametric interval, and by changing parameter the two configurations exchange role. Then there must exist a parameter value corresponding to six tangency points, the most symmetric configuration.

Let us now assume that the parameters $\lambda, m$, and $n$ belong to the given range. Suppose $P_{0}(m)_{x} \geq Q_{0}(n-1)_{x}$. Then $\tau\left(P_{0}(m)\right) \geq \tau\left(Q_{0}(n-1)\right)$. For $m=1$, the fact that $Q_{1}(n-1)$ lies on 5 implies that the right-hand edge $\left[Q_{0}(n-1), Q_{1}(n-1)\right]$ of $\Lambda_{n}^{\text {in }}$ lies to the left of the interior of $\Lambda_{1}^{\text {out }}$ and there is no nontrivial intersection. Assume therefore $m \geq 2$. Since $\tau\left(Q_{0}(n-1)\right)=\tau\left(Q_{1}(n-1)\right)$, and, using (67) and monotonicity, $\tau\left(P_{1}(m)\right)>\tau\left(P_{0}(m)\right)$, we conclude that $\tau\left(P_{1}(m)\right) \geq \tau\left(Q_{1}(n-1)\right)$. Thus $\left[Q_{0}(n-1), Q_{1}(n-1)\right]$ lies to the left of the interior of $\Lambda_{m}^{\text {out }}$ and there is no nontrivial intersection.

If $m=1$, the second inequality in (68) is automatically satisfied, since $P_{1}(0)_{x}=1$. Assume therefore $m \geq 2$, and suppose $P_{1}(m-1)_{x} \leq Q_{1}(n)_{x}$. Then $\tau\left(P_{1}(m-1)\right) \geq$ $\tau\left(Q_{1}(n)\right)$. But $\tau\left(Q_{1}(n)\right)=\tau\left(Q_{0}(n)\right)$ and, using (67) and monotonicity, $\tau\left(P_{1}(m-1)\right)>$ $\tau\left(P_{0}(m-1)\right)$. Thus $\tau\left(P_{0}(m-1)\right) \leq \tau\left(Q_{0}(n)\right)$. We conclude that the left-hand edge $\left[Q_{0}(n), Q_{1}(n)\right]$ of $\Lambda_{n}^{\text {in }}$ lies to the right of the interior of $\Lambda_{m}^{\text {out }}$ and there is no nontrivial intersection.

Defining $n_{+}(m)=\left\lceil n\left(P_{0}(m)\right)\right\rceil$, we know that $n_{+}(m)-1<n\left(P_{0}(m)\right)$ and hence, by monotonicity, the first inequality of (68) holds, not only for $n=n_{+}(m)$, but for all $n \leq n_{+}(m)$. Similarly, if $n_{-}(m)=\left\lceil n\left(P_{1}(m-1)\right\rceil\right.$, we know that $n_{-}(m)<n\left(P_{1}(m-1)\right)$ and hence, by monotonicity, the second inequality of (68) holds, not only for $n=n_{-}(m)$, but for all $n \geq n_{-}(m)$. We conclude that both inequalities hold for $n_{-}(m) \leq n \leq n_{+}(m)$.

From lemma 16, we know that $\lambda\left(n\left(P_{0}(m)\right)-n\left(P_{1}(m-1)\right)\right.$ is bounded below by a positive number. Hence $n_{+}(m)-n_{-}(m)$, which by definition exceeds $n\left(P_{0}(m)-n\left(P_{1}(m-\right.\right.$ $1))-1$, tends to infinity in the limit of vanishing $\lambda$. 


\subsection{Expansion of $Z(m, n)$}

The point $Z(m, n)$ lies at the intersection of the line segments $\left[P_{0}(m), P_{1}(m-1)\right]$ and $\left[Q_{0}(n-1), Q_{1}(n)\right]$. Using linear algebra, we have

$$
Z(m, n)=\frac{1}{w \times z}\left(\begin{array}{ll}
-z_{x} & w_{x} \\
-z_{y} & w_{y}
\end{array}\right)\left(\begin{array}{c}
u \times w \\
v \times z
\end{array}\right)
$$

where

$$
u=P_{0}(m), \quad v=Q_{0}(n-1), \quad w=P_{1}(m-1)-P_{0}(m), \quad z=Q_{1}(n)-Q_{0}(n-1) .
$$

and, for any pair of 2-vectors $a$ and $b$, the cross product is defined as

$$
a \times b=a_{x} b_{y}-a_{y} b_{x} .
$$

Inserting the estimates of lemma 14 and corollary 25, and once again applying the rules of interval arithmetic, we now obtain similar estimates for $Z(m, n)$.

Lemma 18 For $0<\lambda \leq \lambda_{0}$ and $1 \leq m \leq \lambda^{-\nu_{0}}$,

$$
\begin{aligned}
Z(m, n)= & \left(\frac{1}{2}(1+\tau)+\frac{\lambda}{8}\left(7+(1-4 m) \tau^{2}\right)\right. \\
& +\frac{\lambda^{2}}{32}\left(4-(5+16 m) \tau+\left(1-8 m+16 m^{2}\right) \tau^{3}\right)+r_{Z x} \lambda^{5 / 2} \\
& 1+\frac{\lambda}{4}(1-(2 m+1) \tau)+\frac{\lambda^{2}}{16}\left(1+2 m+\left(8 m^{2}+2 m-1\right) \tau^{2}\right) \\
& \left.+\frac{\lambda^{3}}{192}\left(-20+48 m-16 m^{2}+\left(23+6 m-80 m^{2}\right) \tau-\left(3-18 m+96 m^{3}\right) \tau^{3}\right)+r_{Z y} \lambda^{7 / 2}\right),
\end{aligned}
$$

where $\tau=\tau\left(Q_{0}(n)\right)$ and, for $m \geq 3$,

$$
r_{Z x} \in\left[-\frac{1033}{2000}, \frac{2671}{5000}\right], \quad r_{Z y} \in\left[-\frac{5563}{1000}, \frac{7373}{1000}\right] .
$$

For $m=1,2$, we have

$$
\begin{aligned}
Z(1, n)= & \left(\frac{1}{2}(1+\tau)+\frac{\lambda}{8}\left(7-3 \tau^{2}\right)+\frac{\lambda^{2}}{32}\left(4-21 \tau+9 \tau^{3}\right)+r_{Z 1 x} \lambda^{3},\right. \\
& \left.1+\frac{\lambda}{4}(1-3 \tau)+\frac{3 \lambda^{2}}{16}\left(1+3 \tau^{2}\right)+\frac{\lambda^{3}}{64}\left(4-17 \tau-27 \tau^{3}\right)+r_{Z 1 y} \lambda^{4}\right),
\end{aligned}
$$

with

$$
r_{Z 1 x} \in\left[-\frac{1011}{100}, \frac{334}{25}\right], \quad r_{Z 1 y} \in\left[-\frac{4211}{100}, \frac{437}{10}\right]
$$

and

$$
\begin{aligned}
Z(2, n)= & \left(\frac{1}{2}(1+\tau)+\frac{7 \lambda}{8}\left(1-\tau^{2}\right)+\frac{\lambda^{2}}{32}\left(4-37 \tau+49 \tau^{3}\right)+r_{Z 2 x} \lambda^{3}\right. \\
& \left.1+\frac{\lambda}{4}(1-5 \tau)+\frac{5 \lambda^{2}}{16}\left(1+7 \tau^{2}\right)+\frac{\lambda^{3}}{64}\left(4-95 \tau-245 \tau^{3}\right)+r_{Z 2 y} \lambda^{4}\right)
\end{aligned}
$$


with

$$
r_{Z 2 x} \in\left[-\frac{506}{25}, \frac{331}{20}\right], \quad r_{Z 2 y} \in\left[-\frac{9517}{100}, 150\right]
$$

The proof is given in appendix A.6.

\subsection{Disk areas}

In this section we complete the proof of theorem $\mathrm{B}$. The regular fixed point $Z(m, n)$ of $L$ is a periodic point of the map $F$, with period $t(m, n)=4(m+n)-1$, see equation (60). Let $A_{m, n}$ be the area of the cell (stability ellipse) of $Z(m, n)$; furthermore, for fixed $m$, let $A_{m}$ be the total area of the ellipses associated with orbits of $Z(m, n), n=n_{-}(m), \ldots, n_{+}(m)$. Thus

$$
A_{m}=\sum_{n=n_{-}(m)}^{n_{+}(m)} A_{m, n} t(m, n) \quad t(m, n)=4(m+n)-1 .
$$

Finally, we denote by $A^{\text {reg }}$ the total area associated with the regular orbits:

$$
A^{\mathrm{reg}}=\sum_{m=1}^{M} A_{m}
$$

Our goal in this section is to prove that the $\lambda \rightarrow 0^{+}$limits of $A_{m}$ and $A^{\text {reg }}$ exist, and to derive explicit expressions for the limiting areas.

From theorem 17, we know that the ellipse associated with $Z(m, n)$ is tangent to one of the lines $\mathbf{1}$ or $\mathbf{5}^{1}$, and so the area of the ellipse is just $\pi$ times the square of the $\mathcal{Q}$-metric distance from $Z(m, n)$ to the tangent point on the closer line. Using the inner product $\mathcal{Q}$ defined in equation (44), the distance between a point $Z$ and a line segment $[P, Q]$ is given by

$$
\sqrt{\mathcal{Q}(U, U)-\frac{\mathcal{Q}(U, V)}{\mathcal{Q}(V, V)}}, \quad U=Z-P, \quad V=Q-P .
$$

For the area of disks tangent to the line 1, we apply this formula with $Z=Z(m, n), P=$ $(0,1), Q=(1,1)$ to obtain the expression

$$
A_{m, n}^{(0)}=\pi\left(1-\frac{\lambda^{2}}{4}\right)\left(Z(m, n)_{y}-1\right)^{2}
$$

For the disks tangent to $\mathbf{5}^{1}$, on the other hand, we use $P=(g, 1), Q=\left(1, g^{\prime \prime}\right)$ (cf. equations (11) and (14)), to obtain (writing $Z=\left(Z_{x}, Z_{y}\right)$ )

$$
A_{m, n}^{(1)}=\pi\left(1-\frac{\lambda^{2}}{4}\right)\left(1-Z_{y}+\left(1-2 Z_{x}\right) \lambda-\left(1-3 Z_{y}\right) \lambda^{2}-\left(1-Z_{x}\right) \lambda^{3}-Z_{y} \lambda^{4}\right)^{2} .
$$

The above formulae give us a straightforward way of calculating

$$
A_{m, n}=\min \left\{A_{m, n}^{(0)}, A_{m, n}^{(1)}\right\}
$$


for given $m, n, \lambda$. Supplemented by Taylor expansion with rigorous bounds on the remainders, the same formulae also provide the machinery for our asymptotic calculations in the limit $\lambda \rightarrow 0^{+}$.

\subsection{Bounds for the disk areas}

To obtain formulae for the disk areas $A^{(0)}(m, n)$ and $A^{(1)}(m, n)$, we insert $Z(m, n)$ from lemma 18 into (71) and (72), respectively, combining the remainder intervals using the rules of interval arithmetic [12] (secs. E.10,E.10A). We state the results as a lemma.

Lemma 19 For $0<\lambda \leq \lambda_{0}$ and $1 \leq m \leq \lambda^{-\nu_{0}}$,

$$
\begin{aligned}
& A_{m, n}^{(0)}=\frac{\pi}{16}(1-(2 m+1) \tau)^{2} \lambda^{2}+r_{A 0} \lambda^{5 / 2}, \\
& A_{m, n}^{(1)}=\frac{\pi}{16}(1-(2 m-3) \tau)^{2} \lambda^{2}+r_{A 1} \lambda^{5 / 2}
\end{aligned}
$$

where $\tau=\tau\left(Q_{0}(n)\right)$ and, for $m \geq 3$,

$r_{A 0} \in\left[-\frac{6347}{100000}, \frac{183}{6250}\right], \quad r_{A 1} \in\left[-\frac{7091}{100000}, \frac{2857}{50000}\right], \quad r_{A 1}-r_{A 0} \in\left[-\frac{859}{50000}, \frac{3761}{100000}\right]$.

For $m=1$, only $A^{(0)}(1, n)$ is relevant, with

$$
r_{A 0} \in\left[-\frac{221}{6250}, \frac{1179}{100000}\right]
$$

and, for $m=2$,

$$
r_{A 0} \in\left[-\frac{393}{2000}, \frac{787}{20000}\right], \quad r_{A 1} \in\left[-\frac{7859}{1000000}, \frac{7859}{1000000}\right], \quad r_{A 1}-r_{A 0} \in\left[-\frac{63}{2000}, \frac{943}{5000}\right] .
$$

For fixed $m$, the fixed points $Z(m, n)$ are lined up along the line segment $\left[P_{0}(m), P_{1}(m-\right.$ $1)$ ] in order of decreasing $n$, with $Z\left(m, n_{+}(m)\right)$ just to the right of $P_{0}(m)$ and $Z\left(m, n_{-}(m)\right)$ just to the left of $P_{1}(m-1)$ (see figure 9). Regarded as a continuous function of $n$, $A^{(0)}(m, n)$ increases from zero monotonically with decreasing $n$ while $A^{(1)}(m, n)$ decreases to zero monotonically. Thus there exists a value $n=n_{\text {mid }}(m)$ such that

$$
A^{(0)}\left(m, n_{\text {mid }}(m)\right)=A^{(1)}\left(m, n_{\text {mid }}(m)\right),
$$

and hence

$$
\min \left\{A^{(0)}(m, n), A^{(1)}(m, n)\right\}= \begin{cases}A^{(1)}(m, n) & n_{-}(m) \leq n \leq n_{0}(m)-1 \\ A^{(0)}(m, n) & n_{0}(m) \leq n \leq n_{+}(m)\end{cases}
$$

where

$$
n_{0}(m)=\left\lceil n_{\text {mid }}(m)\right\rceil
$$



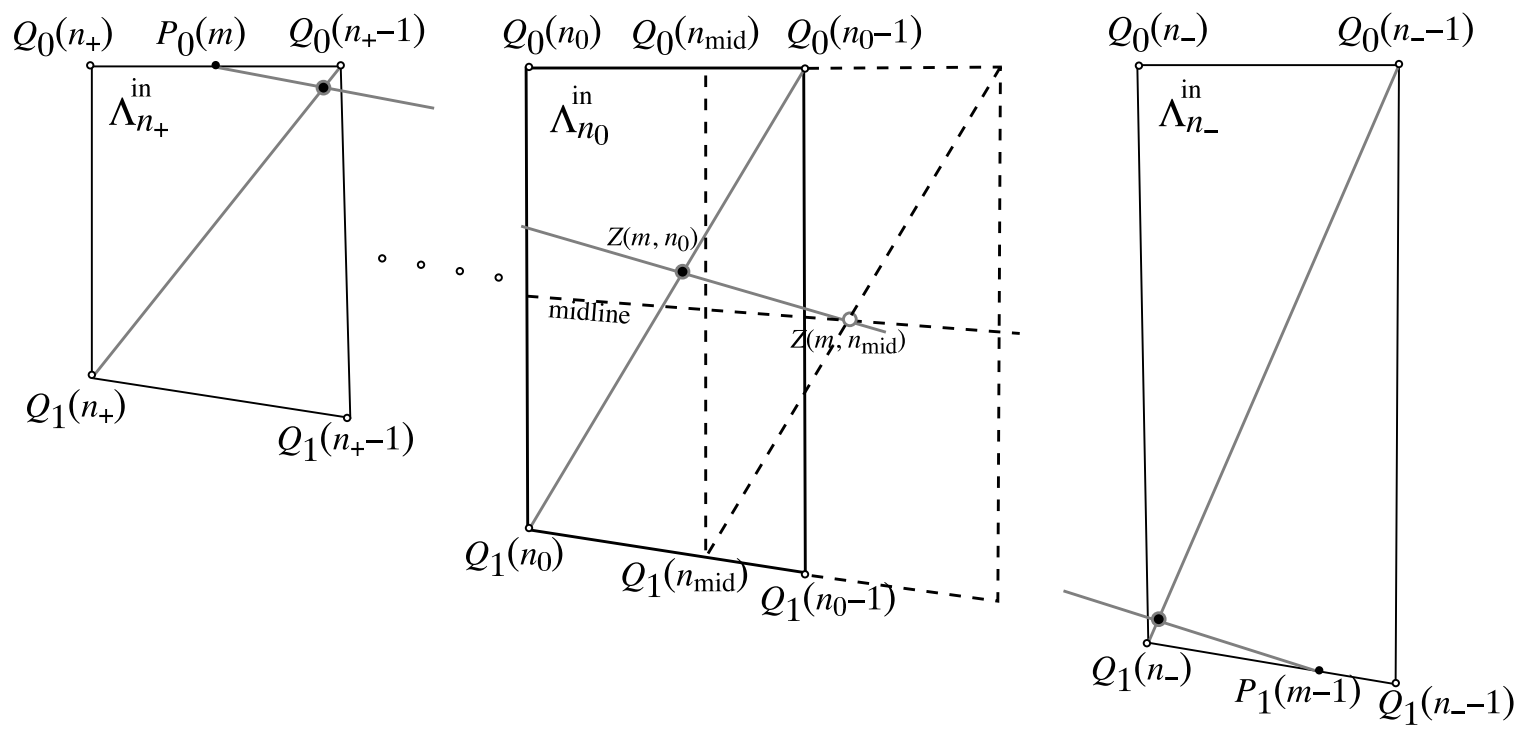

Figure 9: Sketches of the atoms $\Lambda_{n_{+}}^{\text {in }}, \Lambda_{n_{0}}^{\text {in }}$, and $\Lambda_{n_{-}}^{\text {in }}$. The real number $n_{\text {mid }}$ is defined by the condition that $Z\left(m, n_{\text {mid }}\right)$ lies on the 'mid-line' equidistant (in the $\mathcal{Q}$-metric) from the upper and lower boundaries.

To locate $\tau_{\text {mid }}=\tan \left(\frac{\pi}{4}-2 n_{\text {mid }} \theta\right.$ ) (see equation (64) $)$, we equate $A^{(0)}(m, n)$ and $A^{(1)}(m, n)$ in (73) and (74) and solve the resulting quadratic equation in $\tau$ to get (discarding the irrelevant root)

$$
\tau_{\text {mid }}=\frac{1+\sqrt{1+\frac{8}{\pi}(2 m-1) \lambda^{1 / 2}\left(r_{A 1}-r_{A 0}\right)}}{2(2 m-1)} .
$$

Next we have

Lemma 20 The quantity $A^{(0)}(m, n)-A^{(1)}(m, n)$, considered as a function of $\tau$ for fixed $m \in[2, M]$, vanishes at

$$
\tau_{\text {mid }}=\frac{1}{2 m-1}+r_{\text {mid }} \lambda^{1 / 2}
$$

where, for $m \geq 3$,

$$
r_{\text {mid }} \in\left[-\frac{2189}{100000}, \frac{599}{25000}\right]
$$

and, for $m=2$,

$$
r_{\text {mid }} \in\left[-\frac{1003}{25000}, \frac{601}{5000}\right]
$$

Lemma 20 is easily proved using the inequalities (valid for $0 \leq x, y, \leq 1$ and $-x \leq z \leq y$ )

$$
1-x \leq \sqrt{1+z} \leq 1+y / 2 .
$$

An immediate (and useful) consequence of this lemma are the bounds

$$
\tau_{\text {mid }}<\frac{2}{5}
$$




$$
\begin{aligned}
& \left|(2 m-1 \pm 2) \tau_{\text {mid }}-1\right|<1, \\
& \left|A^{(0)}(m, n)\right|<\frac{1}{4} \lambda^{2}, \quad n_{\text {mid }} \leq n \leq n_{+}, \\
& \left|A^{(1)}(m, n)\right|<\frac{1}{4} \lambda^{2}, \quad n_{-} \leq n \leq n_{\text {mid }} .
\end{aligned}
$$

\subsection{Total disk area}

We are now in a position to write an exact formula for the sum of disk areas within a single atom $\Lambda_{m}^{\text {out }}$ :

$$
\begin{aligned}
A_{1} & =\sum_{n=1}^{n_{+}(1)} A^{(0)}(1, n) t(1, n), \\
A_{m} & =\sum_{n=n_{-}(m)}^{n_{0}(m)-1} A^{(1)}(m, n) t(m, n)+\sum_{n=n_{0}(m)}^{n_{+}(m)} A^{(0)}(m, n) t(m, n),
\end{aligned}
$$

where the 'midpoint' $n_{0}(m)$ was defined in (175). To calculate the leading behaviour for $\lambda$ tending to zero, we note that the terms in (81) proportional to $4 m-1$ are negligible in the limit. Specifically,

$$
\begin{aligned}
3 A_{1} & <3 N A^{(0)}(1,1)<12 N \lambda^{2}<\frac{3}{25} \lambda^{1 / 2}, \\
(4 m-1) A_{m} & <4 m N A^{(0)}\left(m, n_{\text {mid }}\right)<\frac{1}{10} \lambda^{1 / 2}, \quad m \geq 2,
\end{aligned}
$$

where we have applied (78) as well as

$$
\lambda<10^{-4}, \quad m \leq \lambda^{-1 / 4}, \quad \lambda N \leq \frac{\pi \lambda}{8 \theta}<1 .
$$

The next step toward the derivation of an asymptotic formula is to insert the estimates (73) and (74) of lemma 19, to obtain

$$
\begin{aligned}
& A_{1}=\frac{\pi \lambda^{2}}{4}\left(\sum_{n=1}^{n_{+}(1)}(1-3 \tau)^{2} n\right)+r_{A} \lambda^{1 / 2} \\
& A_{m}=\frac{\pi \lambda^{2}}{4}\left(\sum_{n=n_{-}(m)}^{n_{0}(m)-1}(1-(2 m-3) \tau)^{2} n+\sum_{n=n_{0}(m)}^{n_{+}(m)}(1-(2 m+1) \tau)^{2} n\right)+r_{A} \lambda^{1 / 2}
\end{aligned}
$$

where $\tau=\tau\left(Q_{0}(n)\right)$ and

$$
\left|r_{A}\right|<\frac{1}{5}
$$

Next, we replace the sum over $n$ by an integral over $\eta$ using the Euler-Maclaurin formula:

$$
A_{1}=\frac{\pi \lambda^{2}}{4} \int_{1}^{n_{+}} d n n(1-3 \tau)^{2}+\frac{\pi \lambda^{2}}{8}\left(4+\left(1-3 \tan \eta_{+}\right)^{2} n_{+}(1)\right)+\left(r_{E M 0}+r_{A}\right) \lambda^{1 / 2},
$$




$$
\begin{aligned}
A_{m}=\frac{\pi \lambda^{2}}{4} & \left(\int_{n_{-}}^{n_{0}-1}(1-(2 m-3) \tau)^{2} n d n\right)+\frac{\pi \lambda^{2}}{4}\left(\int_{n_{0}}^{n_{+}}(1-(2 m+1) \tau)^{2} n d n\right) \\
& +\frac{\pi \lambda^{2}}{8}\left(\left(1-(2 m-3) \tan \eta_{-}\right)^{2} n_{-}+\left(1-(2 m-3) \tan \left(\eta_{0}-2 \theta\right)\right)^{2}\left(n_{0}-1\right)\right) \\
& +\frac{\pi \lambda^{2}}{8}\left(\left(1-(2 m+1) \tan \eta_{0}\right)^{2} n_{0}+\left(1-(2 m+1) \tan \eta_{+}\right)^{2} n_{+}\right) \\
& +\left(r_{E M 0}+r_{E M 1}+r_{A}\right) \lambda^{1 / 2}
\end{aligned}
$$

where, for $1 \leq m \leq \lambda^{-1 / 4}$,

$$
\begin{aligned}
r_{E M 0} & \leq \frac{\pi \lambda^{3 / 2}}{8} \int_{n_{0}}^{n_{+}} d n\left|\frac{d}{d n}\left(n\left(1-(2 m+1) \tan \left(\frac{\pi}{4}-2 n \theta\right)\right)^{2}\right)\right| \\
& \leq \frac{\pi \lambda^{3 / 2}}{8}\left[\int_{n_{0}}^{n_{+}} d n(1-(2 m+1) \tau)^{2}+4 \theta(2 m+1) \int_{n_{0}}^{n_{+}} d n n|1-(2 m+1) \tau| \sec ^{2} \eta\right] \\
& \leq \frac{\pi \lambda^{3 / 2}}{8}\left(N+4 \theta N^{2}(2 m+1)\right)<\frac{1}{7}
\end{aligned}
$$

and an analogous expression for $r_{E M 1}$ for $m>1$.

As a final simplification, we change the integration variable from $n$ to $\eta$, defined in equation (64), changing the integration limits to the values $\eta=\cot ^{-1}(2 m+1), \cot ^{-1}(2 m-$ $1)$, and $\cot ^{-1}(2 m-3)$. We state the result in the form of a lemma, which refers to the cut-off parameter values (63).

Lemma 21 For $0<\lambda \leq \lambda_{0}$, we have

$$
\begin{aligned}
& A_{1}=I(2,1,2)+r \lambda^{1 / 2} \\
& A_{m}=I(m+1, m, m+1)+I(m, m-1, m-1)+r \lambda^{1 / 2}, \quad 2 \leq m \leq \lambda^{-\nu_{0}}
\end{aligned}
$$

where

$$
I(a, b, c) \stackrel{\text { def }}{=} \frac{\pi}{4} \int_{\cot ^{-1}(2 a-1)}^{\cot ^{-1}(2 b-1)}((2 c-1) \tan \eta-1)^{2}(\pi / 4-\eta) d \eta
$$

and $|r|<\frac{4}{5}$.

Proof. The contribution to $r$ of the remainder terms in (85) is bounded in magnitude by $1 / 2$. Moreover, the endpoint terms in (85), for both $m=1$ and $m>1$, are bounded by $\frac{\pi}{2} N \lambda^{2}$ and so (using $N \lambda<1$ ) the magnitude of their contribution to $r$ is bounded by $1 / 50$.

The change of variables produces a factor

$$
\frac{\lambda^{2}}{4 \theta^{2}}=\frac{\sin ^{2}(\theta)}{\theta^{2}} \in\left[1-\theta^{2} / 3,1\right] \subset\left[1-10^{-4} \lambda^{1 / 2}, 1\right] .
$$

Since each of the integrals over $\eta$ is bounded by $\frac{1}{2}\left(\frac{\pi}{4}\right)^{3}<\frac{1}{2}$, we see that the total contribution of the variable change to $r$ is bounded in magnitude by $10^{-4}$. 
Finally, we can estimate the shifts in the integration limits using

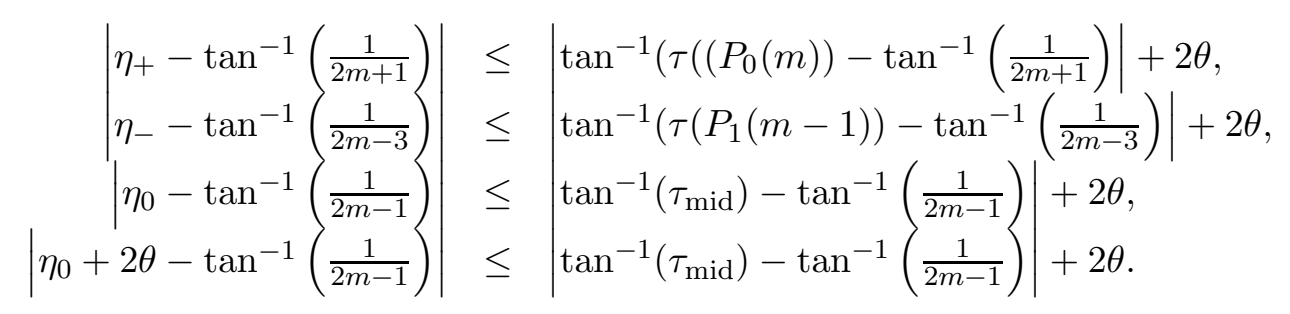

Using lemmas 20 and 24 and the inequality (valid for $x>0, y>0$ )

$$
\tan ^{-1}(x+y)-\tan ^{-1}(x)=\tan ^{-1}\left(\frac{y}{1+x(x+y)}\right)<\tan ^{-1}(y)<y,
$$

it is straightforward to calculate bounds for the right-hand expressions. We find that he contribution to $r$ of the shifts in the integration limits is bounded by $\frac{1}{4}$. Adding up the various contributions to $|r|$, we get the stated upper bound.

Now we are ready to sum up the disk areas from the orbits of all regular fixed points, thus completing the proof of theorem B.

Theorem 22 In the limit $\lambda \rightarrow 0^{+}$, the total area $A^{\mathrm{reg}}(\lambda)$ of the islands of the regular periodic orbits of $F$ converges to the positive quantity

$$
A^{\mathrm{reg}}(0)=I(2,1,2)+\sum_{m=2}^{\infty}(I(m+1, m, m+1)+I(m, m-1, m-1)) .
$$

Proof. It suffices to show that

$$
A^{\mathrm{reg}}(\lambda)=I(2,1,2)+\sum_{m=2}^{\mu(\lambda)}(I(m+1, m, m+1)+I(m, m-1, m-1))+O\left(\lambda^{1 / 4}\right) .
$$

where $I$ is given by lemma 21 and $\mu(\lambda)=\left\lfloor\lambda^{-1 / 4}\right\rfloor$. The partial sum

$$
\sum_{m=2}^{\mu(\lambda)} A_{m}(\lambda)
$$

is already in the desired form, since the integrals are $\lambda$-independent and we have $\left\lfloor\lambda^{-1 / 4}\right\rfloor$ remainder terms, each bounded by the same constant times $\lambda^{1 / 2}$.

The remaining terms,

$$
\sum_{m=\mu(\lambda)+1}^{M(\lambda)} A_{m}(\lambda)
$$

are bounded by the $(\mathcal{Q}$-metric) area of the triangle

$$
\left[P, P_{0}(\mu(\lambda)), P_{1}(\mu(\lambda))\right], \quad P=\left(\frac{1+2 \lambda-\lambda^{2}-\lambda^{3}}{2-\lambda^{2}}, 1\right),
$$


multiplied by the longest return time, $4(M(\lambda)+N(\lambda))-1$. Inserting our estimates for the atomic vertices, the triangular area is

$$
\begin{aligned}
& \frac{1}{2} \sqrt{1-\lambda^{2} / 4}\left(P_{0}(\mu(\lambda))_{x}-P_{x}\right)\left(1-P_{1}(\mu(\lambda))_{y}\right) \\
& =\frac{1}{2}\left(1+O\left(\lambda^{2}\right)\right)\left(\frac{1}{2(2 M(\lambda)+1)}+O\left(\lambda^{3 / 2}\right)\right)\left(\frac{\lambda}{2 M(\lambda)-1}+O\left(\lambda^{5 / 2}\right)\right)=O\left(\lambda^{3 / 2}\right)
\end{aligned}
$$

while the upper bound on the period is $O\left(\lambda^{-1}\right)$. Thus the upper bound on the sum of terms with $m>\mu(\lambda)$ is $O\left(\lambda^{1 / 2}\right)$.

\subsection{Numerical evaluation of area formula}

Numerical integration gives the following results:

\begin{tabular}{|c|l|}
$m$ & \multicolumn{1}{|c|}{ Area $A_{m}$} \\
\hline 1 & 0.04394252102495575454 \\
2 & 0.027915747684440153071 \\
3 & 0.0043390573122902285760 \\
4 & 0.0011842200753144612564 \\
5 & 0.00044544206984605774866 \\
6 & 0.00020336198417268636974 \\
7 & 0.00010566669827864850788 \\
8 & 0.0000602301047692083869367
\end{tabular}

For asymptotically large $m$, the integrands can be expanded in powers of $\eta$ and integrated term by term to yield

$$
A_{m}=\frac{\pi^{2}}{48 m^{4}}+\frac{\pi(\pi-1)}{24 m^{5}}+O\left(m^{-6}\right)
$$

Summing the numerical integrals over the first 2000 values of $m$ gives

$$
\sum_{m=1}^{2000} A_{m}=0.0783220277996 .
$$

This is approximately 36 percent of the area in the square outside the fixed-point disk.

\section{Covering}

In the previous sections we defined the return map $L$ of the domain $\Lambda$, and then we identified a prominent sub-domain of $\Lambda$, namely the union of the regular atoms of $L$. In the following theorem we show that the set of regular atoms is an appropriate surface of section for the orbits of $F$ outside the main island $\mathcal{E}$. In so doing, we will complete the proof of theorem A in the introduction. 
Theorem 23 The images under $F$ of the regular atoms of the return map $L$ cover all of $\Omega \backslash \mathcal{E}$, apart from a set of area $O\left(\lambda^{2}\right)$.

Proof. All metric considerations refer to the $\mathcal{Q}$-metric (44). We begin to deal with the area of the region complementary to $\mathcal{E}$. The $\mathcal{Q}$-area of the square is

$$
\mathcal{A}^{\Omega}=\sqrt{1-\frac{\lambda^{2}}{4}}=1+O\left(\lambda^{2}\right)
$$

The area of the ellipse $\mathcal{E}$ is $\pi r^{2}$, where $r$ is the $\mathcal{Q}$-distance between the fixed point $(1 /(2-$ $\lambda), 1 /(2-\lambda))$ and the point of tangency $T_{0}=((1+\lambda) / 2,1)$. This is

$$
\mathcal{A}^{\mathcal{E}}=\frac{2-3 \lambda+\lambda^{3}}{8-4 \lambda}=\frac{\pi}{4}(1-\lambda)+O\left(\lambda^{2}\right)
$$

Thus, the area outside $\mathcal{E}$ is

$$
\mathcal{A}^{\Omega \backslash \mathcal{E}}=1-\frac{\pi}{4}+\frac{\pi}{4} \lambda+O\left(\lambda^{2}\right)
$$

Now we consider the dynamics, reverting to the representation (17). First, we calculate the area covered by the images of the regular atoms $\Lambda_{m}^{\text {out }}$ under the outer map $\bar{L}^{\text {out }}$. These atoms are polygons whose vertices are listed in appendix A.1.

In the first-order area calculation, we can expand formulae (52) and (53) in Taylor series about $\lambda=0$, keeping only the lowest two terms and an $O\left(\lambda^{2}\right)$ remainder. For $m=1$, the quadrilateral $\Lambda_{1}^{\text {out }}$ reduces to a right triangle $[(\lambda+2 / 3,1),(1,1),(1,1-\lambda)]$ with area $\lambda / 6+O\left(\lambda^{2}\right)$. Since the transit time under $F$ is 7 (theorem 11) we get a contribution to the overall area sum of

$$
\frac{7}{6} \lambda+O\left(\lambda^{2}\right)
$$

For $m=2$, the hexagon $\Lambda_{2}^{\text {out }}$ reduces to a quadrilateral with vertices $\left\{\left(\frac{3}{5}+\lambda, 1\right)\right.$, $\left.\left(\frac{2}{3}+\lambda, 1\right),(1,1-\lambda),\left(\frac{2}{3}+\lambda, 1-\frac{\lambda}{3}\right)\right\}$, and hence area $\lambda / 15$. Multiplying by the transit time of 11 , we get

$$
\frac{11}{15} \lambda+O\left(\lambda^{2}\right)
$$

The remaining domains $\Lambda_{m}^{\text {out }}$ are the quadrilaterals $\left[P_{0}(m), P_{0}(m-1), P_{1}(m-1), P_{1}(m)\right]$, $L^{\text {out }}$-symmetric about the respective axes $\left[P_{0}(m), P_{1}(m-1)\right]$. We use the formulae (50) and (51) to calculate the area of $\Lambda_{m}^{\text {out }}$ as

$$
\begin{aligned}
\mathcal{A}_{m}^{\text {out }} & =\sqrt{1-\lambda^{2} / 4}\left(P_{0}(m)-P_{1}(m)\right) \times\left(P_{1}(m)-P_{1}(m-1)\right) \\
& =\frac{\lambda^{4} \cos ((2 m-1) \theta)}{8 \prod_{k=-1}^{1} \sin ((2 m+(2 k-1)) \theta)} .
\end{aligned}
$$

Expanding the right-hand side in powers of $\theta$, we have

$$
\mathcal{A}_{m}^{\text {out }}=\frac{\lambda^{4}\left(1+m^{2} \theta^{2} r(\theta, m)\right)}{8 \theta^{3}(2 m-3)(2 m-1)(2 m+1)},
$$


where, as we prove in appendix A.8, $r(\theta, m)$ is uniformly bounded above and below for $3 \leq m \leq M, 0<\theta \leq \sin ^{-1}(\lambda / 2)$. Multiplying by the transit time $4 m+3$ and summing over $m$ gives

$$
\begin{gathered}
\sum_{m=3}^{M} \frac{4 m+3}{(2 m-3)(2 m-1)(2 m+1)}=\frac{7}{20}-\frac{\lambda}{\pi}+O\left(\lambda^{2}\right), \\
\sum_{m=3}^{M} \frac{m^{2}(4 m+3)}{(2 m-3)(2 m-1)(2 m+1)}=\frac{\pi}{4 \lambda}+O(\log \lambda),
\end{gathered}
$$

and hence

$$
\sum_{m=3}^{M(\lambda)}(4 m+3) \mathcal{A}_{m}^{\text {out }}=\frac{7}{20} \lambda+O\left(\lambda^{2}\right) .
$$

Combining the results for all $m$, the area contribution is

$$
\mathcal{A}^{\text {out }}=\frac{9}{4} \lambda+O\left(\lambda^{2}\right)
$$

Second, we compute the area covered by the orbits of the regular atoms in $\Lambda^{\text {in }}$, under the inner map $\bar{L}^{\text {in }}$. Here again we start with exact formulae for the vertices of the regular atoms given in appendix A.1, and the expansions of lemma 14, Each $\Lambda_{n}^{\text {in }}$ is a quadrilateral $\left[Q_{0}(n), Q_{0}(n-1), Q_{1}(n-1), Q_{1}(n)\right]$ with $L^{\text {out }}$ symmetry axis $\left[Q_{0}(n-1), Q_{1}(n)\right]$ and the area is calculated to be [12]

$$
\begin{aligned}
\mathcal{A}_{n}^{\text {in }} & =\sqrt{1-\lambda^{2} / 4}\left(Q_{0}(n-1)-Q_{0}(n)\right) \times\left(Q_{0}(n)-Q_{1}(n)\right) \\
& =\frac{1}{2} \tau\left(1+\tau^{2}\right) \lambda^{2}-\frac{1}{8}\left(1+4 \tau+4 \tau^{2}+4 \tau^{3}+3 \tau^{4}\right)+r_{A} \lambda^{4},
\end{aligned}
$$

where $\eta$ and $\tau$ were defined in (64), and $r_{A}$ is a function of $\lambda$ and $\tau$ which satisfies $r_{A}<29$. Next we sum the atom areas multiplied by the transit times $4(n-1)$ (theorem 6), and use the Euler-Maclaurin formula to turn it into an integral:

$$
\begin{aligned}
\mathcal{A}^{\text {in }} & =\sum_{n=1}^{N(\lambda)} 4(n-1) \mathcal{A}_{n}^{\text {in }} \\
& =\frac{1}{2} \int_{0}^{\frac{\pi}{4}} d \eta\left(\frac{\pi}{4}-\lambda-\eta\right)\left(4\left(\tau+\tau^{3}\right)-\left(1+4 \tau+4 \tau^{2}+4 \tau^{3}+3 \tau^{4}\right) \lambda\right)+O\left(\lambda^{2}\right) \\
& =1-\frac{\pi}{4}+\frac{\pi-9}{4} \lambda+O\left(\lambda^{2}\right) .
\end{aligned}
$$

Here we omit the detailed handling of error terms (Euler-Maclaurin endpoint and remainder terms, change of integration variable, adjustment of integration limits), which parallels that encountered in the calculation of disk areas in section 3. Details may be found in [12].

Now the regular atoms of $L$ are the intersections of the 'in' and 'out' atoms - see equation (59). The mismatch between the union of the two sets of atoms is $O\left(\lambda^{3}\right)$, from 
theorems 6] and 11, while the respective transit times are $O\left(\lambda^{-1}\right)$. Hence the area covered by the images of the regular atoms of $L$ is $\mathcal{A}^{\text {in }}+\mathcal{A}^{\text {out }}+O\left(\lambda^{2}\right)$. Combining the above area expressions gives us the desired identity

$$
\mathcal{A}^{\Omega}=\mathcal{A}^{\mathcal{E}}+\mathcal{A}^{\text {out }}+\mathcal{A}^{\text {in }}+O\left(\lambda^{2}\right) .
$$

Although the irregular atoms of $L$ do contribute to the total area, the proof of theorem 23 avoids the great difficulty of deriving bounds for their return times.

\section{$7 \quad$ Extension of our results}

We consider, briefly and informally, some extensions of our results.

The regularity of the limit $\lambda \rightarrow 0^{+}$depends on the very simple dynamics at $\lambda=0$, for which there is a single atom. There is one dominant fixed point, while all other cycles disappear at $\lambda=0$, and are arranged in a hierarchy that affords a perturbative analysis in the small parameter $\theta$. Some asymptotic features depend on the product of $\theta$ and the return time of relevant domains, so that large return times lead to an unavoidable nonuniformity in the convergence to the limit. However, this problem remains tractable, due to the regular arrangement of the atoms.

Similar features are present in the limit $\lambda \rightarrow 0^{-}$, so we expect similar phenomena. For $-1<\lambda<0$, there are three symbols: $\iota(x, y) \in\{0,1,2\}$. However, the atom $\Omega_{0}$ is a single point (the origin) and can be neglected. The atom $\Omega_{1}$ is again a quadrilateral with area $O(1)$, while $\Omega_{2}$ is a triangle with area $O(\lambda)$, which plays the same role as the atom $\Omega_{0}$ in the positive $\lambda$ case.

For $-1<\lambda<0$, the basic cycles are

\begin{tabular}{lllcl}
$t$ & code & denominator & numerators & radius \\
\hline 1 & $(\overline{1})$ & $2-\lambda$ & $(1)$ & $x_{0}(\lambda)$ \\
2 & $(\overline{1,2})$ & $4-\lambda^{2}$ & $(4+\lambda, 2(1+\lambda))$ & $1-x_{0}(\lambda)$
\end{tabular}

The fixed point is determined by the same rational function as for the $\lambda \geq 0$ case. The 2 cycle is different; however, changing the sign of $\lambda$, together with a reflection with respect to the centre of $\Omega$, reproduces the same behaviour to first order in $\lambda$. The symmetry properties of these cycles are also unchanged.

The linked sector maps of the 1 - and 2-cycles for $\lambda<0$ are shown in figure 10. Compared to the case $\lambda>0$, there is now a tighter connection between the two sectors, which results in shorter transit times. As for $\lambda>0$, one constructs the transit maps $L^{\text {in,out }}$, and identifies a two-parameter family of regular fixed points with periods

$$
t(m, n)=4(m+n)-3
$$




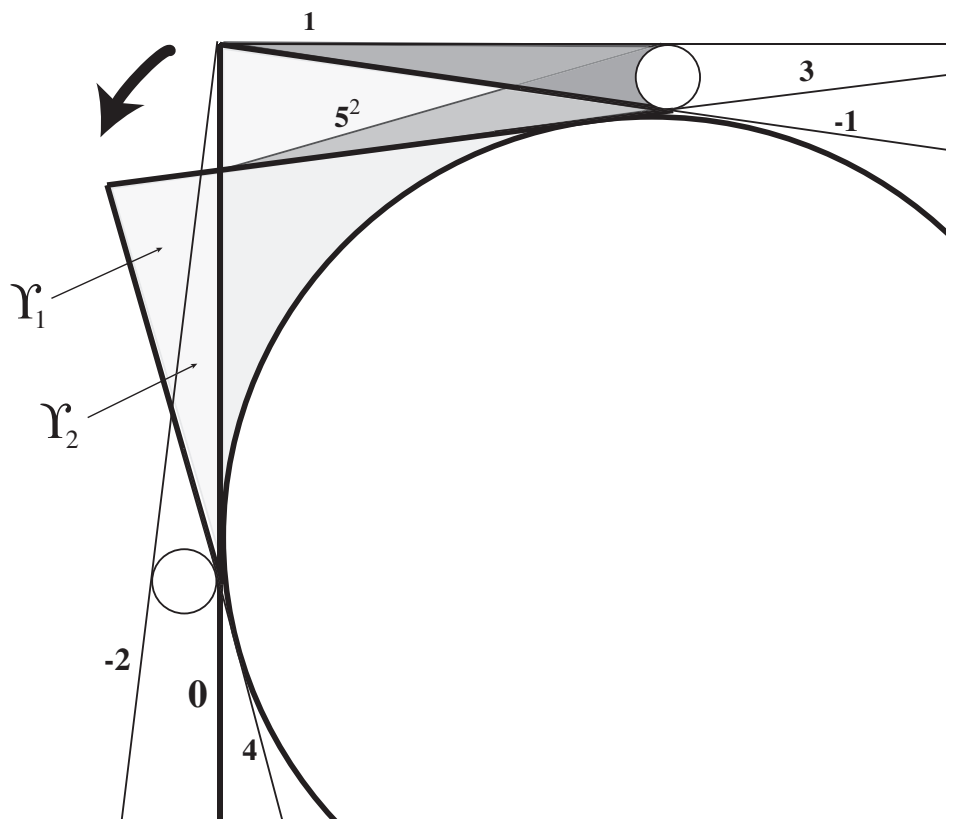

Figure 10: The linked sector maps of the 1-cycle and the 2-cycle for a small negative value of the parameter $\lambda$. The corresponding sectors $\Sigma$ and $\Sigma^{\prime}$ are represented in light and dark grey, respectively. Under the action of the map $F^{4}$, both sectors turns counterclockwise. The points in $\Sigma$ that do not end up in $\Sigma^{\prime}$ (under $F$ ) comprise the triangle $\Upsilon_{1}$; those that do comprise the quadrilateral $\Upsilon_{2}$.

and symbolic codes

$$
\iota^{(n, m)}=\left(\overline{1^{4 n-1},(2,1)^{2 m-1}}\right) \quad n \geq 1, \quad m \geq 1,
$$

to be compared with (60) and (61), respectively. As before, there are anomalous cycles of total area $O(\lambda)$, which interact with the above structure in regions of phase space of area $O\left(\lambda^{2}\right)$. The main cycle of this kind is a fixed point located in the North-West corner of the square $\Omega$.

Returning to the limit $\lambda \rightarrow 0^{+}$, we recall that the mismatch between the sector maps of the 1 and 2-cycles generates a collection of small irregular domains, which were neglected in our perturbative analysis. The dynamics over these domains is determined by the interaction among certain periodic orbits lying outside the sector $\Sigma$.

As an example, let us consider the turnstile associated to the domain $\Upsilon_{1}$ (figure 6). Under the action of $G \circ F^{-4}$, the left triangle comprising the turnstile is mapped to the triangle $\Phi=\langle\mathbf{0}, \mathbf{7}, \mathbf{8}\rangle$, of area $O\left(\lambda^{3}\right)$, lying between the atom $\Lambda_{1}^{\text {out }}$ and the boundary $\mathbf{0}$ of $\Lambda$ (cf. the first equation in (37) $)$.

The domain $\Phi$ belongs to the first regular atom of $L^{\text {in }}$. The partition of $\Phi$ into atoms under $\bar{L}^{\text {out }}$ is shown in figure 11 for two parameter values approaching zero from above. The low-transit-time atoms (all symmetric) belong to three families, coloured blue, red, and green in the figure, and the picture suggests convergence to an asymptotic regime, 


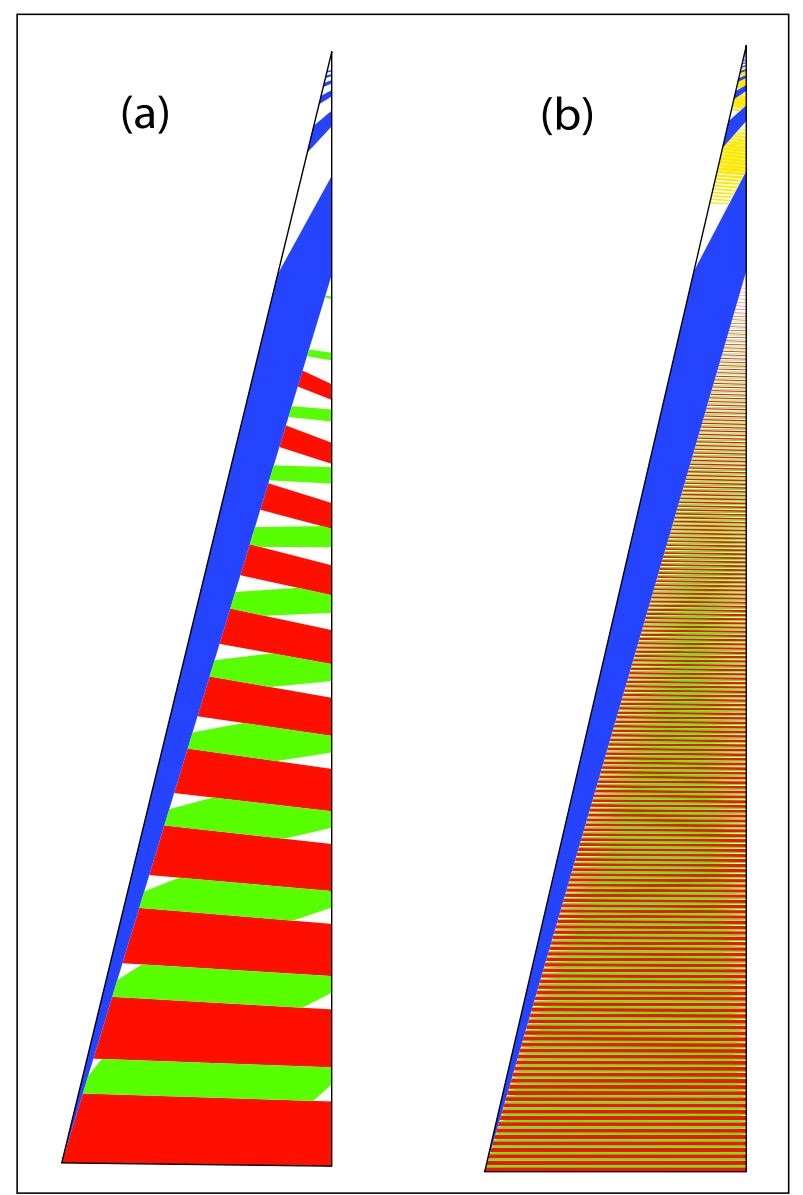

Figure 11: (a) The irregular domain $\Phi$ on the eastern boundary of $\Lambda_{1}^{\text {out }}$, showing three families of atoms. Here $\lambda=\frac{1}{64}$. (b) Same as (a), but with $\lambda=\frac{1}{1000}$. In both cases the horizontal and vertical dimensions have been rescaled to make the structure of the partition visible.

although the convergence seems slow. The atoms' respective codes and transit times are:

\begin{tabular}{|c|c|c|}
\hline Colour & Code & Transit Time \\
\hline \hline Blue & $\left(1(1101101010)^{2 m-1} 110111\right)$ & $20 m-3$ \\
\hline Red & $\left(1(110)^{4 m} 111\right)$ & $12 m+4$ \\
\hline Green & $\left(1(110)^{4 m-1}(1101101010)(110)^{4 m} 111\right)$ & $24 m+11$ \\
\hline
\end{tabular}

We see that these codes shadow two periodic codes $(\overline{110})$ and $(\overline{1101101010})$. These correspond to a 3 - and a 10-cycle lying outside $\Sigma$, whose cells have area $O\left(\lambda^{2}\right)$ and $O\left(\lambda^{4}\right)$, respectively.

In addition to $\lambda=0$, there is trivial dynamics (a single atom) also for $\lambda= \pm 1$ (rational rotation number $\rho=1 / 6,1 / 3)$, and for these values we expect the near-rational dynamics to be broadly similar to that considered in this paper. By contrast, the dynamics for all other rational rotation numbers is highly non-trivial. The simplest cases correspond to quadratic irrational $\lambda$-values (eight in all), for which the phase space is tiled by infinitely many periodic cells which admit an exact renormalization [23]. Preliminary investigations 
for $\lambda=(\sqrt{5}-1) / 2(\rho=1 / 5)$ indicate that the construct of linked sector maps can only serve as a local model for near-rational dynamics; the global properties result from complex interactions between different local models. We have observed a highly nonuniform convergence to the asympotic regime, due to the presence of unbounded return times in the limiting rational dynamics. This problem requires further investigation.

\section{Appendix}

\section{A.1 Rules of interval arithmetic with rational rounding}

Suppose we have rational lower and upper bounds on a set of real quantities $r_{1}, r_{2}, \ldots, r_{n}$ and we wish to obtain a similar bound on a rational function $R$ of these quantities. We can do so by assigning to the quantity $r_{i}$ the closed interval $\left[a_{i}, b_{i}\right]$ where $a_{i} \leq r_{i} \leq b_{i}$ are the given bounds. If $r$ is a rational number, then we assign to it the closed interval $[r, r]$. We then proceed to deduce a bounding interval for $R$ by performing, in any convenient order, a sequence of elementary arithmetic operations, taken from the following list:

(i) $\left[a_{1}, b_{1}\right]+\left[a_{2}, b_{2}\right]=\left[a_{1}+a_{2}, b_{1}+b_{2}\right]$,

(ii) $\left[a_{1}, b_{1}\right] \times\left[a_{2}, b_{2}\right]=\left[\min \left\{a_{1} a_{2}, a_{1} b_{2}, b_{1} a_{2}, b_{1} b_{2}\right\}, \max \left\{a_{1} a_{2}, a_{1} b_{2}, b_{1} a_{2}, b_{1} b_{2}\right\}\right]$,

(iii) For $a b>0, \quad[a, b]^{-1}=\left[\min \left\{\frac{1}{a}, \frac{1}{b}\right\}, \max \left\{\frac{1}{a}, \frac{1}{b}\right\}\right]$,

(iv) For $a \geq 0, b>0, n \geq 0$ or $a<0, b \geq 0, n \geq 0, \quad[a, b]^{n}=\left[\min \left\{a^{n}, b^{n}\right\}, \max \left\{a^{n}, b^{n}\right\}\right]$,

$(v)$ For $a>0, b>0, n>0, \quad[a, b]^{-n}=\left([a, b]^{n}\right)^{-1}$.

The result is an exact, but obviously non-unique, pair of bounds for $R$.

Since multiple application of these rules can lead to prohibitively large denominators for the resulting rational bounds, it is convenient to round off after every elementary operation. The lower and upper bounds are rounded down (resp. up) to the nearest rational number with a power of 10 in the denominator and a specified number of significant digits in the numerator. Any common factors in numerator and denominator can be divided out. For example, with the 4-digit rational rounding used in this article, the interval $[\pi / 2, \pi]$ would be rounded to $[157 / 100,3142 / 10000]$.

The Mathematica interval functions used to obtain rigorous bounds throughout this article are listed in sec. E.1 of [12].

\section{A.2 Proof of $P_{k}(m)$ estimates in lemma 14}

The initial terms in the stated formulae for $P_{0}(m)$ and $P_{1}(m)$ result from the Taylor expansion of (50) and (51) up to second order in the $x$-component and third order in 
the $y$-component (in the latter case, the zeroth order term is trivially equal to unity). Our task is to establish the stated numerical bounds on the remainder coefficients $r_{i}, i=$ $0 x, 1 x, 1 y, 01 x, 10 y, 11 y$.

We begin with

$$
r_{0 x}=(2 m+1) P_{0}(m)_{x}-1-m-(2 m+1) \lambda+\frac{m}{6}(m+1) \lambda^{2} .
$$

Defining auxiliary variables $u$ and $v$ by the expansion formulae

$$
\begin{gathered}
x \cot (x)=1-\frac{x^{2}}{3}-(1+u) \frac{x^{4}}{45}, \\
\theta=\sin ^{-1}\left(\frac{\lambda}{2}\right)=\frac{\lambda}{2}+(1+v) \frac{\lambda^{3}}{48} .
\end{gathered}
$$

Inserting these expansions reduces $P_{0}(m)_{x}$, hence $r_{0 x}$, to ratios of polynomials in $\lambda, m, u$, and $v$. The numbers of terms is in these polynomials is enormous, but the expressions are easily manipulated with computer assistance. Moreover, all but a handful of terms have sufficiently high powers of $\lambda$ that they contribute insignificantly to the final estimates (recall our cut-off condition $\lambda<\lambda_{0}=10^{-4}$ ). The detailed calculations have been relegated to the electronic supplement [12] (E.7.1).

To obtain a bounding interval for $r_{0 x}$, we use the following bounds [12] (E.7.1) for our variables:

$$
\lambda \in\left[0,10^{-4}\right], \quad m \in\left[2, \lambda^{-1 / 4}\right], \quad u \in[-3 / 2500,3 / 1000], \quad v \in\left[0,2 \times 10^{-8}\right] .
$$

Each monomial of the form $\pm \lambda^{a} m^{b} u^{d} v^{f}$ is assigned a single bounding interval using the rules of interval arithmetic (see appendix A.1) with fixed precision rational rounding down (resp. up) of lower (resp. upper) bounds. The monomial bounds are then be rigorously combined, again using interval arithmetic, to give the final bounds for $r_{0 x}$ stated in the lemma. The same technique was used to establish [12] (E.7.1-2) the stated bounds on the other remainder factors in the lemma.

We have implemented the process described above with the aid of Mathematica functions involving only elementary logical and arithmetic manipulations of symbols and integers. The specific procedures are listed in the Electronic Supplement [12], sec.E.1. Note that while our estimates are rigorous, they are not optimal, and can easily be changed by permuting the order in which monomial bounds are combined.

A potential difficulty of the method comes from the fact that the upper bound for $m$ grows as $\lambda^{-1 / 4}$, which is numerically unbounded for $\lambda$ in the chosen range. The problem is avoided by a simple trick based on the fact that all polynomial expressions which we wish to bound have only monomials of the form $\pm \lambda^{a} m^{b} \cdots$ where $b$ is either negative or no greater than $4 a$. In the first case we use $m^{b} \in\left[0,2^{b}\right]$, and in the second case we use $\lambda^{a} m^{b} \in\left[0, \lambda_{0}^{a-b / 4}\right]$. If the original rational function is not in this form, we try, if possible, to make it acceptable by dividing both numerator and denominator by a common power of $m$. 


\section{A.3 Proof of $Q_{k}(n)$ estimates in lemma 14}

Taylor expansion of (54) and (55) has been carried out with respect to $\lambda$ with $\tau$ held fixed. The remainders, expressed as rational functions of $\lambda$ and $\tau$ are

$$
r_{2 x}=\frac{\mathcal{N}_{2 x}}{\mathcal{D}_{2 x}}, \quad r_{3 x}=\frac{\mathcal{N}_{3 x}}{\mathcal{D}_{3}}, \quad r_{3 y}=\frac{\mathcal{N}_{3 y}}{\mathcal{D}_{3}},
$$

For $r_{2 x}$, we have

$$
\begin{aligned}
\mathcal{N}_{2 x}= & -64+64 \tau^{2}+32 \lambda+32 \tau \lambda-32 \tau^{2} \lambda-32 \tau^{3} \lambda+32 \lambda^{2}-16 \tau \lambda^{2}-40 \tau^{2} \lambda^{2}+ \\
& 16 \tau^{3} \lambda^{2}+8 \tau^{4} \lambda^{2}-24 \tau \lambda^{3}+16 \tau^{2} \lambda^{3}+8 \tau^{3} \lambda^{3}-4 \tau \lambda^{4}+10 \tau^{2} \lambda^{4}-4 \tau^{3} \lambda^{4}-2 \tau^{4} \lambda^{4}+ \\
& \sqrt{4-\lambda^{2}}\left(32-32 \tau^{2}-16 \lambda-16 \tau \lambda+16 \tau^{2} \lambda+16 \tau^{3} \lambda-12 \lambda^{2}+8 \tau \lambda^{2}+\right. \\
& \left.16 \tau^{2} \lambda^{2}-8 \tau^{3} \lambda^{2}-4 \tau^{4} \lambda^{2}-2 \lambda^{3}+5 \tau \lambda^{3}-6 \tau^{3} \lambda^{3}+2 \tau^{4} \lambda^{3}+\tau^{5} \lambda^{3}\right), \\
\mathcal{D}_{2 x}= & 32 \lambda^{2}\left(-8 \tau+2 \lambda^{2} \tau+\sqrt{4-\lambda^{2}}\left(\lambda-\lambda \tau^{2}\right)\right) .
\end{aligned}
$$

Application of interval bounds to these expressions runs into a serious difficulty: the denominator has a zero (barely!) within the allowed range $0 \leq \tau \leq 1$, namely at

$$
\tau_{1}=\frac{2 \sqrt{4-\lambda^{2}}-4-\lambda^{2}}{\lambda \sqrt{4-\lambda^{2}}}=\frac{\lambda}{4}+O\left(\lambda^{3}\right) .
$$

Fortunately, the numerator shares the same zero, and we can divide both $\mathcal{N}_{2 x}$ and $\mathcal{D}_{2 x}$ by the factor $\left(\tau-\tau_{1}\right)$. The result takes the form

$$
r_{2 x}=\frac{\mathcal{N}_{2 x}^{\prime}}{\mathcal{D}_{2 x}^{\prime}}\left(2 \sqrt{4-\lambda^{2}}-4+\lambda^{2}\right)^{-5}
$$

where $\mathcal{D}_{2 x}^{\prime}$ and (especially) $\mathcal{N}_{2 x}^{\prime}$ are lengthy polynomials in $\lambda, \tau$, and $\sqrt{4-\lambda^{2}}$.

To apply the interval methods used successfully in appendix A.2, it turns out that we need to use a high-order estimate for the square root, namely

$$
\sqrt{4-\lambda^{2}}=2-\frac{\lambda^{2}}{4}-\frac{\lambda^{4}}{64}-\frac{\lambda^{6}}{512}-\frac{5 \lambda^{8}}{16384}-\frac{7 \lambda^{10}}{131072}-\frac{21 v \lambda^{12}}{2097152} .
$$

Inserting the interval estimates

$$
v \in\left[1, \frac{1117}{1000}\right], \quad \tau \in[0,1], \quad \lambda \in\left[0, \lambda_{0}\right],
$$

in each of the 3 polynomial components of $r_{2 x}$ in (90) and applying the rules of appendix A.1 to combine them, we arrive, finally at the stated bounds. Applying the same techniques to $r_{3 x}, r_{3 y}, r_{4 x}, r_{5 x}$, we get the remaining estimates in lemma 14. Details of the calculations may be found in [12], sec. E.7.3. 


\section{A.4 $\tau$ estimates}

In addition to the contents of lemma 14 concerning the cartesian coordinates of the $\Lambda_{m}^{\text {out }}$ vertices, we will also need information relating to their $\tau$-coordinates (see (64)). Estimates for the latter may be obtained by combining the formulae of lemma 14. In the following lemma, the remainder terms are rigorously bounded [12] using the same techniques discussed in appendices A.2 and A.3.

Lemma 24 For $\lambda \leq \lambda_{0}=10^{-4}$ and $2 \leq m \leq \lambda^{-1 / 4}$, we have the expansions

$$
\begin{aligned}
\tau\left(P_{0}(m)\right)= & \mu^{-1}+\frac{\lambda}{4}\left(5+2 \mu^{-1}+\mu^{-2}\right) \\
& +\frac{\lambda^{2}}{48}\left(24+(57-16 m(m+1)) \mu^{-1}+12 \mu^{-2}+3 \mu^{-3}\right)+r_{0 \tau} \lambda^{3},
\end{aligned}
$$

where $\mu=2 m+1, r_{0 \tau} \in\left[-\frac{4541}{1000}, \frac{3087}{1000}\right]$, and

$$
\begin{aligned}
\tau\left(P_{1}(m)\right)= & \mu^{\prime-1}+\frac{\lambda}{4}\left(1+2 \mu^{\prime-1}+5 \mu^{\prime-2}\right) \\
& +\frac{\lambda^{2}}{48}\left(24+(129-16(m+2)(m+3)) \mu^{\prime-1}+60 \mu^{\prime-2}+75 \mu^{\prime-3}\right)+r_{1 \tau} \lambda(392)
\end{aligned}
$$

where $\mu^{\prime}=2 m-1, r_{1 \tau} \in\left[-\frac{633}{20}, \frac{731}{50}\right]$. Moreover,

$$
\tau\left(P_{0}(1)\right)=\frac{1}{3}+\frac{13}{9} \lambda+\frac{19}{27} \lambda^{2}+r_{01 \tau} \lambda^{3}
$$

with $r_{01 \tau} \in\left[\frac{383}{2500}, \frac{2819}{1000}\right]$.

As a corollary, we get bounds on $\tau\left(Q_{0}(n)\right)$ restricted to a single value of $m$.

Corollary 25 For $\lambda \in\left[0,10^{-4}\right], m \in\left[3, \lambda^{-1 / 4}\right]$,

$$
m \tau\left(Q_{0}(n)\right)=r_{\tau} \in\left[\frac{2}{5}, \frac{1001}{1000}\right] \text {. }
$$

Proof. For those atoms $\Lambda_{n}^{\text {in }}$ which intersect $\Lambda_{m}^{\text {out }}$, we have the inequalities

$$
\tau\left(P_{1}(m-1)\right) \geq \tau\left(Q_{0}(n)\right)=\tan \left(\gamma\left(Q_{0}(n-1)\right)-2 \theta\right) \geq \frac{\tau\left(P_{0}(m)\right)-\tan (2 \theta)}{1+\tan (2 \theta)} .
$$

where, for $\lambda<10^{-4}$,

$$
\tan (2 \theta)=\frac{\lambda \sqrt{4-\lambda^{2}}}{2-\lambda^{2}}<\left(1+10^{-8}\right) \lambda .
$$

From lemma 24 it is not difficult to derive [12]

$$
\tau\left(P_{0}(m)\right)=\frac{1}{2 m+1}+\left(\frac{5}{4}+r_{0}\right) \lambda, \quad r_{0} \geq-\frac{1467}{2 \times 10^{6}}
$$




$$
\tau\left(P_{1}(m)\right)=\frac{1}{2 m-1}+\left(\frac{1}{4}+r_{2}\right) \lambda, \quad r_{2}<\frac{153}{500} .
$$

With the inequalities (valid in the cut-off regime)

$$
\frac{m}{2 m+1} \geq \frac{3}{7}, \quad \frac{m}{2 m-3} \leq 1, \quad 0 \leq \lambda m \leq \frac{1}{1000}
$$

we obtain

$$
\frac{2}{5}<m \tau\left(Q_{0}(n)\right)<\frac{1001}{1000}
$$

\section{A.5 Proof of lemma 16}

The differences in the $n$-coordinate are related to those in $\tau$ by

$$
n_{2}-n_{1}=\frac{1}{2 \theta}\left(\tan ^{-1}\left(\tau_{1}\right)-\tan ^{-1}\left(\tau_{2}\right)\right)=\frac{1}{2 \sin ^{-1}(\lambda / 2)} \tan ^{-1}\left(\frac{\tau_{1}-\tau_{2}}{1+\tau_{1} \tau_{2}}\right) .
$$

From lemma 24 we have

$$
\begin{aligned}
& \tau\left(P_{1}(m)\right)-\tau\left(P_{0}(m)\right)=\frac{2}{4 m^{2}-1}+O(\lambda) \\
& \tau\left(P_{1}(m-1)\right)-\tau\left(P_{0}(m-1)\right)=\frac{2}{4 m^{2}-8 m+3}+O(\lambda) \\
& \frac{3}{5} \lambda<\tau\left(P_{0}(m-1)\right)-\tau\left(P_{1}(m)\right)<\frac{17}{10} \lambda .
\end{aligned}
$$

Inserting these estimates in (94) and considering the small-argument behaviour of the inverse trigonometric functions, we obtain the desired relations.

\section{A.6 Proof of lemma 18}

The method of proof is a familiar one. The remainder factors $r_{Z x}$ and $r_{Z y}$ are constructed by combining (69) and (70) with the insertion of $P_{0}(m), P_{1}(m-1), Q_{0}(n-1)$, and $Q_{1}(n)$ from lemma 18. Here care must be taken to express $Q_{0}(n-1)$ in terms of the variable $\tau=\tau\left(Q_{0}(n)\right)$ :

$$
\tau\left(Q_{0}(n-1)\right)=\tan \left(\frac{\pi}{2}-2 n \theta+2 \theta\right)=\frac{\tau+\tan (2 \theta)}{1-\tau \tan (2 \theta)} .
$$

The resulting expressions (see [12] for details) for $r_{Z x}$ and $r_{Z y}$ are enormous polynomials in $\lambda, m, \tau$, and the various remainder factors appearing in the expansion formulae for the atom vertices. Because the $Z(m, n)$ lies within a single atom $\Lambda_{m}^{\text {out }}$, we improve our estimates by replacing $\tau$ by $r / m$, where, from appendix A.5, $r \in[2 / 5,1001 / 1000]$. Application of the rules of interval arithmetic (appendix A.1) then yields the uniform bounds stated in the lemma. 


\section{A.7 Crossover calculation}

We now return to the crossover phenomenon for a more precise treatment. First of all, we observe that the angle between the line segments $\left[P_{0}(m), P_{1}(m)\right]$ and $\left[Q_{0}\left(n\left(P_{0}(m)\right)\right.\right.$, $Q_{1}\left(n\left(P_{0}(m)\right)\right]$ changes sign as one proceeds from right to left in $\Lambda$. For some $m=m^{*}, n=$ $n^{*}=n\left(P_{0}\left(m^{*}\right)\right)$, the segments coincide and the atoms $\Lambda_{m^{*}}^{\text {out }}$ and $\Lambda_{n^{*}}^{\text {in }}$ have their vertical edges nearly parallel. We adopt $m=m^{*}$ as our precise definition of the crossover. By the construction of section 3 , the orientation of the line through $\left[P_{0}(m), P_{1}(m)\right]$ differs from the vertical by a generalized rotation $C^{-4 m}$, while $\left[Q_{0}(n), Q_{1}(n)\right]$ is rotated by $C^{4 n+3}$. Thus crossover corresponds to

$$
C^{4 m^{*}+4 n^{*}+3}= \pm \mathbf{1}
$$

Obviously there are infinitely many solutions, but the relevant one, corresponding to a single crossover as $m$ varies from 1 to $M$, has a negative sign, with $m^{*}$ satisfying

$$
\left(4 m^{*}+4 n\left(P_{0}\left(m^{*}\right)\right)+3\right) \sin ^{-1}\left(\frac{\lambda}{2}\right)=\frac{\pi}{2},
$$

where $n\left(P_{0}(m *)\right)$ is calculated by solving $Q_{0}(n)=X$ for $n$, then setting $X=P_{0}(m *)$. The equation for $m *$ can be solved perturbatively to obtain

$$
\begin{aligned}
m^{*}(\lambda) & =\frac{1}{\sqrt{2 \lambda}}\left(1+\frac{\lambda}{3}+\frac{\lambda^{2}}{120}+\frac{31 \lambda^{3}}{1008}+O\left(\lambda^{4}\right)\right) \\
n^{*}(\lambda) & =\frac{\pi}{4 \lambda}-\frac{1}{\sqrt{2 \lambda}}-\frac{3}{4}-\frac{\sqrt{\lambda}}{3 \sqrt{2}}-\frac{\pi \lambda}{96}-\frac{\lambda^{3 / 2}}{120 \sqrt{2}}+O\left(\lambda^{2}\right)
\end{aligned}
$$

In particular, $m^{*}(\lambda)$ diverges to infinity as $\lambda \rightarrow 0$.

\section{A.8 Proof of $\Lambda_{m}^{\text {out }}$ area estimate}

We are seeking uniform bounds, for $3 \leq m \leq M$ and $0<\theta \leq 10^{-4}$ on

$$
r(\theta, m)=\frac{\theta^{-2} m^{-2} c((2 m-1) \theta)}{s((2 m-3) \theta) s((2 m-1) \theta) s((2 m+1) \theta)}-1,
$$

where

$$
c(x)=\cos (x), \quad s(x)=\frac{\sin x}{x} .
$$

Note that in calculation of bounds we will not be able to benefit from the previous cut-off on $m$.

Following our usual practice, we define auxiliary variables $u$ and $v$ via

$$
c(x)=1-\frac{x^{2}}{2}+\frac{(1-u) x^{4}}{24}, \quad s(x)=1-\frac{x^{2}}{6}+\frac{(1-v) x^{4}}{120} .
$$

Bounds $0 \leq u, v \leq 1002 / 1000$ are straightforward to establish [12] (sec. E.11.1). Insertion of these expressions reduces $r(\theta, m)$ to a ratio of polynomials in the variables $\theta, m, u$, and 
$v$. Although each polynomial can be uniformly bounded above and below using the same methods used throughout this article, there is a serious problem: the denominator range includes zero. On the other hand, numerical evaluation of the denominator shows that it is almost certainly negative definite.

To proceed, we isolate the leading contribution consisting of terms proportional to $m^{k} \theta^{k}$ (for all others, the power of $m$ is less than that of $\theta$ ). There is no $u$ dependence of these terms. With respect to $v$, we make the worst-case assumption, setting it equal to zero for all negative terms and to its maximum value of 1002/1000. The leading part of the denominator then reduces to

$$
\begin{gathered}
\mathcal{D}_{1}(\theta, m)=h\left(m^{2} \theta^{2}\right), \\
h(x)=-1728000+3456000 x-\frac{11513088}{5} x^{2}+1433600 x^{3} \\
+\frac{2328576}{25} x^{4}+\frac{384768768}{3125} x^{5}+\frac{24096096064}{1953125} x^{6} .
\end{gathered}
$$

We need to show that this polynomial is negative definite over the range $0<x<\frac{\pi^{2}}{16}<$ $x_{1}=\frac{6171}{10000}$. Direct application of interval arithmetic fails, as expected, producing a range of values containing zero. On the other hand, the same method, applied to the derivative $h^{\prime}(x)$ produces a pair of positive bounds. Thus $h(x)$ is a monotone increasing function. Since it is negative at both 0 and $x_{1}$ it must be negative everywhere on the interval. Specifically,

$$
\mathcal{D}_{1}(\theta, m) \in[-1728000,-110000]
$$

Meanwhile, the numerator $\mathcal{N}(\theta, m, u, v)$ and the non-leading part of the denominator, $\mathcal{D}_{2}(\theta, m, u, v)$ can be bounded by our original method, obtaining [12] (sec. E.11.1)

$$
\mathcal{N}(\theta, m, u, v) \in[-24730000,27090000], \quad \mathcal{D}_{2}((\theta, m, u, v) \in[-31800000,14920] .
$$

Combining the bounding intervals according to the rules of appendix A.1, we get, finally

$$
r(\theta, m) \in\left[-285, \frac{1301}{5}\right]
$$

\section{References}

[1] R. Adler, B. Kitchens and C. Tresser, Dynamics of nonergodic piecewise affine maps of the torus, Ergod. Th. and Dynam. Sys. 21 (2001) 959-999.

[2] P. Ashwin, Elliptic behaviour in the sawtooth standard map, Phys. Lett. A 232 (1997) 409-416.

[3] P. Ashwin and A. Goetz, Invariant curves and explosion of periodic islands for piecewise isometries, SIAM Journal of Applied Dynamical Systems 4 (2005) 437-458. 
[4] P. Ashwin, W. Chambers, and G. Petrov, Lossless digital filters overflow oscillations: approximations of invariant fractals, Inter. J. Bifur. Chaos 7 (1997) 2603-2610.

[5] N. Bird and F. Vivaldi, Periodic orbits of the sawtooth maps, Physica D 30 (1988) 164-176.

[6] D. Bosio and F. Vivaldi, Round-off errors and p-adic numbers, Nonlinearity 13 (2000) 309-322.

[7] H. Bruin, A. Lambert, G. Poggiaspalla and S. Vaienti, Numerical investigations of a discontinuous rotation of the torus, Chaos 13, (2003), no.2, 558-571.

[8] J. Buzzi, Piecewise isometries have zero topological entropy, Ergod. Th. and Dynam. Sys. 21 (2001) 1371-1377.

[9] L. O. Chua and T. Lin, Chaos in digital filters, IEEE Trans. Circuits and Systems CAS-35 (1988) 648-658.

[10] L. O. Chua and T. Lin, Fractal pattern of second order non-linear digital filters: a new symbolic analysis, Int. J. Cir. Theor. Appl. 18 (1990) 541-550.

[11] A. C. Davies, Nonlinear oscillations and chaos from digital filters overflow, Phil. Trans. R. Soc. Lond. A 353 (1995) 85-99.

[12] J. H. Lowenstein and F. Vivaldi, Electronic Supplement to this article, https://files.nyu.edu/jhl2/public/NearZeroSupp.pdf (2009).

[13] A. Goetz, Dynamics of piecewise isometries, PhD Thesis, University of Chicago (1996).

[14] A. Goetz, Dynamics of a piecewise rotation, Continuous and Discrete Dyn. Sys. 4 (1998) 593-608.

[15] A. Goetz, Dynamics of piecewise isometries, Illinois Journal of Mathematics 44 (2000) $465-478$.

[16] A. Goetz, Stability of piecewise rotations and affine maps, Nonlinearity 14 (2001) 205-219.

[17] A. Goetz, Return maps in cyclotomic piecewise similarities, Dynamical systems 20 (2005) 255-265.

[18] A. Goetz and G. Poggiaspalla, Rotation by $\pi / 7$, Nonlinearity 17 (2004) 1787-1802.

[19] I. S. Gradshteyn and I. M. Ryzhik, Table of integrals series and products. Academic Press, New York (1965).

[20] B. Kahng, Dynamics of symplectic affine maps on tori, PhD Thesis, University of Illinois at Urbana-Champain (2000).

[21] B. Kahng, Dynamics of symplectic piecewise affine elliptic rotation maps on tori, Ergodic Theory and Dynamical Systems 22 (2002) 483-505.

[22] B. Kahng, Singularities of 2-dimensional invertible piecewise isometric dynamics, preprint (2009). (To appear in Chaos.) 
[23] K. L. Kouptsov, J. H. Lowenstein and F. Vivaldi, Quadratic rational rotations of the torus and dual lattice maps, Nonlinearity 15 (2002) 1795-1842.

[24] J. S. W. Lamb and J. A G. Roberts, Time-reversal symmetry in dynamical systems: a survey, Physica D 112 (1998) 1-39.

[25] J. H. Lowenstein, S. Hatjispyros and F. Vivaldi, Quasi-periodicity, global stability and scaling in a model of Hamiltonian round-off, Chaos 7 (1997) 49-66.

[26] J. H. Lowenstein, Aperiodic orbits of piecewise rational rotations of complex polygons with recursive tiling, Dynamical Systems 22 (2007) 25-63

[27] J. H. Lowenstein, K. L. Kouptsov, and F. Vivaldi, Recursive tiling and geometry of piecewise rotations by $\pi / 7$ Nonlinearity 172004 1-25.

[28] J. H. Lowenstein and F. Vivaldi, Embedding dynamics for round-off errors near a periodic orbit, Chaos 10, (2000) 747-755.

[29] I. Niven, Irrational numbers, The Mathematical Association of America, Washington DC (1956).

[30] G. Poggiaspalla, Self-similarity in piecewise isometric systems, Dynamical Systems, 21 (2006) 147-189.

[31] F. Vivaldi and J. H. Lowenstein, Arithmetical properties of a family of irrational piecewise rotations, Nonlinearity 19 (2006) 1069-1097.

[32] C. J. Vowden and B J Vowden, Chaos in digital filters: Identification of all periodic sequences admissible adjacent to zero, Nonlinearity 20 (2007) 975-1006.

[33] C. W. Wu and L. O. Chua, Properties of admissible sequences in a second-order digital filter with overflow non-linearity, Int. J. Cir. Theor. Appl. 21 (1993) 299-307. 
(a)

(c)

III...

(b) 